\title{
Multimorbidity in general practice : adverse health effects and innovative research strategies
}

Citation for published version (APA):

Aarts, S. (2012). Multimorbidity in general practice : adverse health effects and innovative research strategies. [Doctoral Thesis, Maastricht University]. Datawyse / Universitaire Pers Maastricht. https://doi.org/10.26481/dis.20120413sa

Document status and date:

Published: 01/01/2012

DOI:

10.26481/dis.20120413sa

Document Version:

Publisher's PDF, also known as Version of record

\section{Please check the document version of this publication:}

- A submitted manuscript is the version of the article upon submission and before peer-review. There can be important differences between the submitted version and the official published version of record.

People interested in the research are advised to contact the author for the final version of the publication, or visit the DOI to the publisher's website.

- The final author version and the galley proof are versions of the publication after peer review.

- The final published version features the final layout of the paper including the volume, issue and page numbers.

Link to publication

\footnotetext{
General rights rights.

- You may freely distribute the URL identifying the publication in the public portal. please follow below link for the End User Agreement:

www.umlib.nl/taverne-license

Take down policy

If you believe that this document breaches copyright please contact us at:

repository@maastrichtuniversity.nl

providing details and we will investigate your claim.
}

Copyright and moral rights for the publications made accessible in the public portal are retained by the authors and/or other copyright owners and it is a condition of accessing publications that users recognise and abide by the legal requirements associated with these

- Users may download and print one copy of any publication from the public portal for the purpose of private study or research.

- You may not further distribute the material or use it for any profit-making activity or commercial gain

If the publication is distributed under the terms of Article $25 \mathrm{fa}$ of the Dutch Copyright Act, indicated by the "Taverne" license above, 
Multimorbidity in general practice:

Adverse health effects and innovative research strategies

Sil Aarts 
The studies presented in this dissertation were conducted under the auspices of the School for public Health and Primary Care (CAPHRI) and the school for Mental Health and Neuroscience (MHeNS) at Maastricht University. CAPHRI is part of the Netherlands School of Primary Care Research (CaRe), which has been acknowledge since 1995 by the Royal Netherlands Academy of Art and Sciences (KNAW)

(C) Copyright S. Aarts, Maastricht 2012

ISBN 9789461591326

Cover illustration: Ruud Aarts

Book preparation, cover design and printing: Datawyse

All rights reserved. No part of this book may be reproduced or transmitted in any form or by any means, electronic or mechanical, including photocopying, recording, or by any information storage and retrieval system without written permission from the published, except for the inclusion of quotations in a review. 


\section{Multimorbidity in general practice:}

\section{Adverse health effects and innovative research strategies}

\section{Proefschrift}

Ter verkrijging van de graad van doctor aan de Universiteit Maastricht, op gezag van de Rector Magnificus,

Prof. Mr. G.P.M.F. Mols,

volgens het besluit van het college van Decanen,

in het openbaar te verdedigen op vrijdag 13 april om 12.00 uur.

door

Sil Aarts

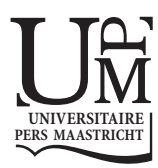




\section{Promotor}

Prof. dr. J.F.M. Metsemakers

Prof. dr. F.R.J. Verhey

\section{Copromotor}

Dr. M. van den Akker

Dr. M.P.J. van Boxtel

\section{Beoordelingscommissie}

Prof. dr. F. Buntinx (voorzitter)

Prof. dr. L. Fratiglioni (Karolinska Institutet, Sweden)

Prof. dr. J. Jolles (Vrije Universiteit Amsterdam)

Prof. dr. G.I.J.M. Kempen

Prof. dr. F. G. Schellevis (NIVEL) 
Be not afraid to progress slowly, be afraid only to stand still

Chinese Wisdom 



\section{Table of Contents}

Chapter 1 General Introduction 9

Chapter 2 Multimorbidity is associated with subjective memory complaints in a 17 large general population of older adults

Chapter 3 Influence of multimorbidity on cognition in a normal aging population: a 12 year follow-up from the Maastricht Aging Study

Chapter 4 The effect of multimorbidity on health related functioning: temporary or persistent? Results from a longitudinal cohort study

Chapter 5 Co-presence of disability and multimorbidity with frailty: an examination of heterogeneity in the frail older population

Chapter 6 Diabetes mellitus type II as a risk factor for depression; a lower than expected risk in a general practice setting

Chapter 7 Exploring medical data to generate new hypotheses: an introduction to data and text mining techniques in epidemiology

Chapter 8 Patterns of multimorbidity: identifying psychiatric and somatic disease combinations using an exploratory analyzing approach

Chapter 9 General Discussion

Chapter 10 Summary

Samenvatting

Dankwoord

About the author

Publications 



\section{CHAPTER 1}

General introduction 


\section{Introduction}

The increase in longevity, the growing number of older people and the decreasing number of newborns, denotes that the populations of most countries in the world are aging rapidly. To date, Europe has the highest proportion of older people in the world. In 2011, the Netherlands had a total population of 16.5 million inhabitants of whom $15.6 \%$ were aged 65 years or older ${ }^{1}$. It has been projected that by the year $2050,24.5 \%$ of the Dutch population will be aged 65 or older ${ }^{1}$. This increase is primarily due to changes in health indicators including improved nutrition and increasing hygiene ${ }^{2}$. Moreover, with advances in preventive and curative medicine, an increasingly large number of patients survive medical conditions that used to be fatal. Because of these developments, the percentage of individuals who are suffering from a chronic disease, a disease of long duration and generally slow progression ${ }^{3}$, has increased substantially. The prevalence of multimorbidity, the occurrence of two or more chronic diseases within a single individual ${ }^{4}$, has also increased significantly the past few decades. Prevalence estimates of multimorbidity ranging from $23 \% 5$ to over $50 \%{ }^{6}$ of the population under study have been reported. Hence, general practitioners (GPs), being the gatekeepers to other health care facilities, are increasingly confronted with individuals who suffer from multiple pathology. In a qualitative study aimed at exploring the views and attitudes of GPs managing patients with multimorbidity, GPs reported to have difficulties with the often complex health situations of patients with multimorbidity ${ }^{7}$. They also reported that they lacked confidence or clinical competence, when confronted with multimorbidity.

Multiple pathology may lead to faster disease progression, to problems in diagnosing new medical conditions and may require more complex therapy and health care ${ }^{8}$. For example, the number of different physicians seen annually by the average patient ranges from 4 physicians to 14 physicians when suffering from one medical condition to respectively five or more medical conditions. These results indicate the significant impact of multimorbidity on the daily practice of health care. Multimorbidity is also well known for its profound effects on patients. Previous research claims that multimorbidity is associated with various adverse health outcomes including activity limitations and decrease in quality of life. These adverse health effects appear to increase with the number of chronic medical conditions. Having multiple medical conditions makes it more challenging for patients to participate effectively in the process of their own care. Multimorbidity might also affects the patient's family, friends and other caretakers since caring may take a lot of time and effort and may require considerable changes in lifestyle.

To properly assess multimorbidity and it's impact on patients, valid general-practice based data regarding multimorbidity and related health factors are indispensable. Increasing our understanding of multimorbidity may not only result in improved health care delivery for patients with multimorbidity but may also give direction to future intervention programs aimed at ameliorating the adverse health effects of multimorbidity. This thesis reports on multimorbidity and several of its adverse health effects and related constructs. In addition, innovative research strategies to detect prevalent patterns of multimorbidity are introduced and applied. 


\section{MULTIMORBIDITY: DEFINITION AND OPERATIONALZIATION}

A major obstacle in studying multimorbidity is the absence of a universally accepted consensus regarding the use of the term multimorbidity. Although there is still an ongoing debate about the terminology and definition of multimorbidity, in this thesis the definition as proposed by van den Akker and colleagues, which has been widely used and referred to will be used. This definition of multimorbidity, i.e. the co-occurrence of two ore more chronic conditions in one individual ${ }^{4}$, views all medical conditions from an overall perspective. This definition differs from co-morbidity, which is defined as the presence of one ore more medical conditions in addition to an index condition?

Based on a bibliometric study in 2005, Fortin and colleagues concluded that despite the high prevalence of multimorbidity, relatively few articles in the medical literature have been devoted to multimorbidity or co-morbidity ${ }^{10}$. Since then multimorbidity has become more prominent in observational scientific research. However, most research is still focused on specific, single diseases, hereby excluding patients with multiple medical conditions.

Research on multimorbidity may be scarce because the term multimorbidity comes with a range of methodological problems ${ }^{11}$. As stated previously, the definition of multimorbidity is the first problem that arises. Secondly, the number and set of diseases on which multimorbidity will be based, is decisive for the prevalence of multimorbidity. In general, a high number of diseases under study will result in a high prevalence of multimorbidity. When only a small number of diseases will be evaluated, a non-realistic picture of the prevalence of multimorbidity is presented. Thirdly, diagnostic criteria and diagnostic procedures that are used for the definition of a chronic medical condition strongly influence the prevalence of multimorbidity. For example, a disease will be identified more frequently if the diagnostic criteria are less strict or when active case-finding is used. Moreover, when a disease symptom (e.g. hypertension) is considered as a chronic medical condition, the prevalence of multiple pathology is also expected to rise. Fourthly, previous studies on multimorbidity are conducted in restricted populations such as nursing home patients or geriatric patients. Research in these subpopulations may provide results that are less representative for the general population. Fifthly, multimorbidity is often assessed by means of self-reported medical status. However, self-reports encompass individual interpretations and expectations and may reflect various underlying causes. Moreover, since the agreement between self-reports and medical records tends to weaken in older age groups and in people with multiple pathology ${ }^{12}$, results from studies using selfreported multimorbidity may be less adequate.

Perhaps the most important methodological issue that arise is the conceptualization of multimorbidity. One of the most widely used methods for the assessment of multimorbidity is a simple disease count; a count of the number of chronic medical conditions in a single individual ${ }^{13,}{ }^{14}$. A variant of the simple disease count is the conditional count, also known as comorbidity; the number of chronic diseases in addition to an index disease. Although both a simple disease count and a conditional count are straightforward and parsimonious, counting the number of diseases within a single individual might be overly simplistic since disease severity is not accounted for. Moreover, 
in a simple disease count as well as in a conditional count a potential strong association between a single disease and a particular outcome may become diluted when intermingled with diseases which do not have an effect on the outcome at hand. Another method that is often used to assess multimorbidity is an index score in which all chronic conditions within an individual are weighted according to their severity (e.g. the Charlson index $\left.{ }^{15}\right)^{16}$. Although this method does account for disease severity, it requires the use of medical records and trained reviewers. Multimorbidity can also be assessed by the relative association between diseases. This method assesses the degree to which a specific combination of diseases (e.g. diabetes mellitus and depression) exceeds a level expected by chance alone (i.e. as if they were independent $)^{8}$. In part, our understanding regarding multimorbidity is limited because studies have used a variety of approaches that all have to be considered in regard to the research aim, study design and study population. Therefore, it is difficult if not unfeasible to compare the results of various studies on multimorbidity.

Another problem that rises when studying multimorbidity is the use of other gerontological constructs such as frailty (i.e. a state of increased vulnerability that may result from decreased physiological reserve ${ }^{17}$ ) and disability (i.e. according to the WHO any restriction or lack of the ability to perform an activity in the manner or within the range considered normal for the individual ${ }^{18}$ ), which are often used interchangeably with multimorbidity to identify the weakest and most vulnerable subset of older adults. The relationship between multimorbidity and these other construct is complex and still poorly understood.

\section{INNOVATIVE RESEARCH STRATEGIES}

The introduction of personal computers in health care has rapidly increased the acquisition and storage of medical information which in turn has resulted in vast amounts of medical data and published medical text (e.g. published research articles stored in Medline). Large medical datasets as well as the large body of medical literature can be potentially relevant in the context of dedicated medical research questions. As a result of the lack of ability to extract new information and insight from these enormous amounts of medical data, medical researchers may be missing out on a significant portion of relevant medical information and knowledge. Therefore, new methods are needed in order to accurately transform these vast amounts of data and text into usable information.

Piatetsky-Shapiro was the first to introduce the term Knowledge Discovery in Databases (KDD) when referring to the process of inferring implicit and hidden patterns into well-grounded and testable hypotheses ${ }^{19,20}$. The primary goal of KDD is to find the unexpected, that is, to discover associations and patterns that may not logically follow from earlier research ${ }^{21}$. KDD can be divided into two different, but related domains: data mining and text mining. Data mining is the process of discovering patterns in large databases in an automatic or semiautomatic manner which can result in new classification schemes, newly generated hypotheses or new predictive models which were previously unknown ${ }^{22-25}$. Text mining refers to the process of analyzing large collections of documents to discover promising new hypotheses ${ }^{26,27}$. Since KDD methods are able to identify meaningful 
patterns in large databases, KDD could be an efficient approach in exploring medical databases for novel patterns of multimorbidity.

\section{Patterns of Multimorbidity}

Knowledge of the patterns of multimorbidity in a given population has important implications for medical research, health care delivery and daily practice. More specifically, a thorough understanding of frequently co-occurring medical conditions might improve our ability to predict future occurring medical conditions and mat provide insight to underlying pathological mechanism. Moreover, health care for and quality of life of patients with multimorbidity might also be improved.

Given the large spectrum of chronic medical conditions one would expect many patterns of multimorbidity (i.e. combinations of co-occurring diseases) which can either have a non-causal association or can share a common pathophysiological cause. However, if we want to investigate all possible existing combinations of co-occurring medical conditions we are confronted with a colossal amount of possible multimorbidity patterns. Therefore, data approaches that can identify meaningful patterns and relations in massive amounts of medical data are required in order to identify novel, potentially relevant medical information.

\section{SUCCESFUL AGING}

The worldwide increase in longevity does not necessarily reflect improvement of health of individuals. If this increase in longevity solely occurs because individuals, instead of dying, become disabled or ill for a longer period of time, then the health of a population has not improved $^{2}$. If a longer life is due to a lower occurrence of ill individuals, the health of a population will improve. Consequently, in the last decades, the focus of medical research and daily practice is on "successful aging" 28 . Successful aging has been defined as a summary of three main components: 1) low probability of disease and disease-related susceptibility, 2) high cognitive and physical functioning, and 3) active engagement with life. As this definition indicates, successful aging is a multidimensional concept, of which the included concepts are all highly intertwined. Several studies included in this thesis focuses on the association between two of the three components of successful aging namely diseases on one side and cognitive and physical functioning on the other.

\section{RESEARCH AIMS AND OBJECTIVES}

The primary aim of this thesis is to advance our knowledge regarding multimorbidity. Expanding our knowledge regarding multimorbidity may improve heath care delivery for individuals with multimorbidity and can facilitate the development of future intervention programs aimed at reducing the adverse health consequences associated with multimorbidity. Gaining knowledge on multimorbidity may be acquired by the use of innovative research strategies. With this specific aim in mind, the following primary aims, with corresponding research questions, were formulated: 
1) to report on several adverse health-related factors and related constructs.

- Is multimorbidity associated with subjective memory complaints?

- What is the longitudinal effect of multimorbidity on cognitive functioning?

- Are the mental and physical problems that accompany multimorbidity temporary or persistent?

- What is the relation between frailty, multimorbidity and disability?

- Is diabetes mellitus type II related to subsequent depression in a general practice based population?

2) to introduce and apply innovative research strategies useful for analyzing large amounts of medical data.

- Can Knowledge Discovery in Databases (KDD) be of use in medical research?

- Which patterns of multimorbidity frequently occur in patients above a chance level?

\section{OUTLINE}

Chapter 2 describes the association between multimorbidity and subjective memory performance in a large community-dwelling population of older adults. Chapter 3 evaluates the association between multimorbidity and objective cognitive performance by making use of the Registration Network family Practice (RNH) and the Maastricht Aging Study (MAAS). Chapter 4 focuses on the association between multimorbidity and functional health. The objective of this study was to evaluate if the changes in mental and physical functioning, caused by multimorbidity, are permanent or temporal. Chapter 5 discusses the relation between frailty, multimorbidity and disability by evaluating their association with several biological markers, nursing home admission and mortality. This study was conducted by means of the Age, Gene/Environment Susceptibility study. Chapter 6 describes the influence of diabetes mellitus type II on subsequent depression in a longitudinal study based on a general practice-based population. Chapter 7 presents a methodological outline of Knowledge Discovery in Databases which can aid the medical research in the process of hypothesis generation. Chapter 8 describes an exploratory analyzing approach in which two selection criteria were combined with an expert evaluation phase in order to identify undiscovered, potential relevant disease combinations in a large general practice-based data set. The general discussion is presented in the last chapter, chapter 9. This chapter recaptures the main findings of the abovementioned studies and discusses the methodological issues. Finally, implications of the findings for clinical practice are presented even as recommendations for future research. 


\section{REFERENCES}

1. Sociale Monitor, Centraal Bureau voor de Statistiek, 2010; Available from: http://statline.cbs.nl/StatWeb/publication/.

2. R.G. Evans, M.L. Barer, and T.R. Marmor. Why are some people healthy and other not? The determinants of health of populations. 1994, New York: Walter de Gruyter, Inc.

3. http://www.who.int/topics/chronic_diseases/en/.

4. M. van den Akker, F. Buntinx, J.F. Metsemakers, S. Roos, and J.A. Knottnerus. Multimorbidity in general practice: prevalence, incidence, and determinants of co-occurring chronic and recurrent diseases. J. Clin. Epidemiol., 1998;51(5):367-375.

5. F.G. Schellevis, J. van der Velden, E. van de Lisdonk, J.T. van Eijk, and C. van Weel. Comorbidity of chronic diseases in general practice. J. Clin. Epidemiol., 1993;46(5):469-473.

6. M. Fortin, G. Bravo, C. Hudon, A. Vanasse, and L. Lapointe. Prevalence of multimorbidity among adults seen in family practice. Ann. Fam. Med., 2005;3(3):223-228.

7. S.M. Smith, S. O'Kelly, and T. O'Dowd. GPs' and pharmacists' experiences of managing multimorbidity: a 'Pandora's box'. Br. J. Gen. Pract., 2010;60(576):285-294.

8. L.G. Glynn, J.M. Valderas, P. Healy, E. Burke, J. Newell, P. Gillespie, and A.W. Murphy. The prevalence of multimorbidity in primary care and its effect on health care utilization and cost. Fam. Pract., 2011;28(5):516-523.

9. A.R. Feinstein and M. Levitt. The role of tonsils in predisposing to streptococcal infections and recurrences of rheumatic fever. N. Engl. J. Med., 1970;282(6):285-291.

10. M. Fortin, L. Lapointe, C. Hudon, and A. Vanasse. Multimorbidity is common to family practice: is it commonly researched? Can. Fam. Physician, 2005;51:244-245.

11. M. van den Akker, F. Buntinx, S. Roos, and J.A. Knottnerus. Problems in determining occurrence rates of multimorbidity. J. Clin. Epidemiol., 2001;54(7):675-679.

12. S. Goebeler, M. Jylha, and A. Hervonen. Self-reported medical history and self-rated health at age 90. Agreement with medical records. Aging clinical and experimental research, 2007;19(3):213-219.

13. R. John, D.S. Kerby, and C.H. Hennessy. Patterns and impact of comorbidity and multimorbidity among community-resident American Indian elders. Gerontologist, 2003;43(5):649-660.

14. J.M. Guralnik. Assessing the impact of comorbidity in the older population. Ann. Epidemiol., 1996;6(5):376-380.

15. D.M. Needham, D.C. Scales, A. Laupacis, and P.J. Pronovost. A systematic review of the Charlson comorbidity index using Canadian administrative databases: a perspective on risk adjustment in critical care research. J. Crit. Care, 2005;20(1):12-19.

16. N.I. Martinez-Velilla and I. Gaminde Inda. Comorbidity and multimorbidity indexes in the elderly patients. Med. Clin. (Barc). 2011;136(10):441-446.

17. L.P. Fried, C.M. Tangen, J. Walston, A.B. Newman, C. Hirsch, J. Gottdiener, T. Seeman, R. Tracy, W.J. Kop, G. Burke, and M.A. McBurnie. Frailty in older adults: evidence for a phenotype. J. Gerontol. A. Biol. Sci. Med. Sci., 2001;56(3):146-156.

18. Disabilities, World Health Organization; Available from: http://www.who.int/topics/disabilities/en/.

19. C. Piatetsky-Shapiro. Knowledge Discovery in Real Databases: A Report on the IJCAI-89 Workshop. Artificial Intelligence Magazine, 1991;11(5):68-70.

20. G. Piatetsky-Shapiro. Data mining and knowledge discovery 1996 to 2005: overcoming the hype and moving from "university" to "business" and "analytics". Data Mining and Knowledge Discovery, 2007;15(1):99-105.

21. R. Agrawal, T. Imielinski, and A. Swami. Database mining: A Performance Perspective. IEEE Transactions on Knowledge and Data Engineering, 1993;5(6):914-925.

22. J.D. Hand. Data mining: statistics and more? The American Statistician, 1998;52:112-118.

23. A.M. Berger and C.R. Berger. Data mining as a tool for research and knowledge development in nursing. Computers, Informatics, Nursing, 2004;22(3):123-131. 
24. J.H. van Bemmel, E.M. van Mulligen, B. Mons, M. van Wijk, J.A. Kors, and J. van der Lei. Databases for knowledge discovery. Examples from biomedicine and health care. Int. J. Med. Inf., 2006;75(34):257-267.

25. I.H. Witten and E. Frank. Data mining: practical machine learning tools and techniques. 2nd. ed. 2005, San Francisco: Elsevier.

26. I. Spasic, S. Ananiadou, J. McNaught, and A. Kumar. Text mining and ontologies in biomedicine: making sense of raw text. Briefings in Bioinformatics, 2005;6(3):239-251.

27. N. Uramoto, H. Matsuzawa, T. Nagano, A. Murakami, H. Takeuchi, and K. Takeda. A text-mining system for knowledge discovery from biomedical documents. IBM Systems Journals, 2004;43(3).

28. J.W. Rowe and R.L. Kahn. Successful aging. Gerontologist, 1997;37(4):433-440. 


\section{CHAPTER 2}

Multimorbidity is associated with subjective memory complaints in a large general population of older adults

Published as

S Aarts, M. van den Akker, K.J. Hajema, A.M. van Ingen, J.F.M. Metsemakers, F.R.J.

Verhey, M.P. J. van Boxtel. International Psychogeriatrics, 2011;23(4):616-624. 


\section{Chapter 2}

\section{ABSTRACT}

\section{Background}

Multimorbidity has been suggested to be predictive of a variety of negative health-related outcomes. The present study was designed to assess the validity of multimorbidity as a predictor of subjective memory complaints.

\section{Methods}

This cross-sectional study was based on data obtained from a postal survey designed by the Public Health Service (Gemeentelijke Gezondheidsdienst, GGD) involving 15,188 persons aged 55 and over living independently in Limburg, the Netherlands. Multivariate logistic regression analyses, adjusted for potentially important covariates, were performed to evaluate the predictive value of self-reported multimorbidity on three outcomes related to subjective memory complaints.

\section{Results}

Multimorbidity was predictive of subjective memory complaints. The ability of multimorbidity to predict subjective memory complaints was positively influenced by age. Moreover, multimorbidity predicted the degree of worrying about memory complaints in people who perceived themselves as forgetful. Multimorbidity was also an important predictor for reporting a larger increase in these subjective memory complaints during the last year. In this latter case, multimorbidity had more prognostic capability in men than in women. Psychological distress was a significant predictor for all three subjective memoryrelated outcome measures.

\section{Conclusions}

In our sample, which was representative of the Dutch population, multimorbidity was predictive of subjective memory complaints. The prognostic value of multimorbidity differed between men and women and between age groups. 


\section{INTRODUCTION}

Subjective memory complaints, or the feeling that one's ability to remember and to retrieve information is not as good as it used to be, are common complaints among middle-aged and elderly persons. One study using a large sample of almost 2000 participants aged 24 to 86 years indicated that nearly 40 percent considered themselves to be forgetful ${ }^{1}$. In a sample of 500 older people between 50 and 90 years of age, 57 percent of the respondents were slightly concerned about their forgetfulness, and over 32 percent were very concerned ${ }^{2}$.

Whether subjective memory complaints are indicative of Mild Cognitive Impairment (MCI) or dementia has been studied extensively, but no clear-cut conclusions have emerged so $\mathrm{far}^{3}$. Some studies have demonstrated that self-perceived forgetfulness is indeed predictive of cognitive decline and dementia ${ }^{4,5}$. It has been argued that a person's belief about his or her own memory performance is an important moderator of changing memory functioning because it can affect memory performance through cognitive, affective, and motivational routes ${ }^{6}$. Other studies have found no association between subjective memory complaints and objective memory decline and argued that these complaints are merely related to depressive feelings and anxiety ${ }^{7,8}$. Moreover, earlier research suggested that subjective feelings of memory impairment are more closely related to personality traits (i.e., feelings of inferior personal competence and capacities as well as tendencies toward phobic or obsessive/compulsive reactions) than to actual memory performance in normal elderly people?

Although subjective memory complaints are not necessarily directly related to objective memory decline ${ }^{7}$, the extent and impact of these self-perceived memory problems could in any case be significant. Previous research has demonstrated that, in older individuals, subjective memory complaints were associated with a lower quality of life ${ }^{10}$. Moreover, in a study conducted in a general practice-based population, results indicated that the presence of subjective memory complaints increased the cost of health care utilization by $60 \%$ over three years ${ }^{11}$. These findings underscore the impact of subjective memory complaints on a person's daily life and on society as a whole. Hence, it is important to gain more insight into the predictive factors contributing to subjective memory complaints. Investigating these potential contributing factors may contribute to the development of a risk profile for elderly patients with self-perceived forgetfulness.

Multimorbidity, a term that describes the co-occurrence of two or more chronic medical conditions within one person ${ }^{12}$, has been indicated as one factor that might contribute to the presence of subjective memory complaints. Prior research has suggested that morbidity status (i.e., morbidity and multimorbidity) might be predictive of objective memory decline ${ }^{13}$. A variety of medical conditions, including diabetes mellitus ${ }^{14}$ and cardiovascular diseases ${ }^{15}$ have already been associated with decreased memory performance. Given that multimorbidity is the rule rather than the exception among older individuals and that patients suffering from multimorbidity use health care services more frequently than patients suffering from a single chronic condition ${ }^{16}$, the impact of multimorbidity on society is substantial.

Although subjective memory complaints have been an important topic in recent gerontological and neuropsychological research, multimorbidity as a predictive factor for 
subjective memory complaints has, to our knowledge, never been evaluated. The first aim of the current study was to evaluate the ability of multimorbidity to predict the presence of subjective memory complaints. The second aim was to investigate whether multimorbidity was prognostic for the degree of worrying about these memory complaints. The final aim was to determine if multimorbidity was predictive of an increase in memory complaints during the previous year. The results of the present study could help clinicians to identify subgroups of older people who are at risk of developing subjective memory complaints, which may be mediated by poor morbidity status.

\section{METHODS}

\section{Study Design}

This study was based on data from a cross-sectional postal survey designed by the Public Health Service (Geneeskundige Gezondheidsdienst, GGD Zuid Limburg and GGD Limburg-Noord) and was sent to a representative sample (of the municipal registrar's office) of people aged 55 and over living in the province of Limburg, the Netherlands. This questionnaire was comprised of questions concerning health status, lifestyle and special care needs.

\section{Study Population}

In 2008, more than 21,000 community-dwelling persons aged 55 and over were sent a questionnaire, which was completed and returned by 15,188 persons (response rate of 72.3 percent). All participants were living in the province of Limburg, the Netherlands.

\section{Measures}

\section{Independent variables}

Morbidity (i.e., the occurrence of one disease within a patient) and multimorbidity (i.e., the co-occurrence of two or more diseases within one patient) were conceptualized by a simple disease count ${ }^{17}$. This count incorporated self-reported medical conditions that had been diagnosed by a medical doctor (see Appendix). Based on these medical conditions, four distinct groups of respondents were formed: 1) people without any of the medical conditions of interest, 2) people who suffered from one medical condition (i.e., morbidity), 3) people who suffered from two medical conditions (i.e., multimorbidity 2), and 4) people who suffered from three or more medical conditions (i.e., multimorbidity $\geq 3$ ). The latter group was formed to determine if the degree of multimorbidity mattered.

\section{Dependent variables}

Memory complaints were probed using three questions. The first question was, 'Do you perceive yourself as forgetful?' and required a yes or no answer. Participants who replied affirmatively $(n=3,976)$ also rated the degree of worrying about their subjective memory complaints on a 5-point scale ranging from 1 ('not at all') to 5 ('a great deal'). Based on this question, two groups were identified, those who perceived themselves as forgetful but did not worry about it (a rating of 1,2 or 3 on the 5 -point scale was required; $n=3,527$ ) and 
those who considered themselves as forgetful and were also worried about their forgetfulness (a rating of 4 or 5 on the 5-point scale was required; $n=446$ ). The third question asked the respondent if his or her memory complaints had increased during the last year. Again, five answers were possible, ranging from 1 ('not at all') to 5 ('a great deal'). Again, two groups were created. The first group contained participants who perceived themselves as forgetful and whose forgetfulness had not increased much during the last year (a rating of 1, 2 or 3 on the 5-point scale was required; $n=3,586$ ). The other group consisted of participants who perceived themselves as forgetful and whose forgetfulness had increased during the last year (a rating of 4 or 5 on the 5 -point scale was required; $n=254$ ).

\section{Confounders}

Potential confounders that were taken into account included sex, age (grouped in two categories: people aged 55 to 69 and people aged 70 and over), education level (low, medium or high), current smoking status (smoker/nonsmoker), alcohol intake (excessive drinker: yes/no; excessive was defined as more than 21 glasses per week for men and more than 14 glasses per week for women), living arrangements (living alone: yes/no), exercise (persons who meet the Dutch Healthy Exercise Standard (Nederlandse Norm Gezond Bewegen) versus persons who did not meet this standard) ${ }^{18}$, healthy eating habits (persons who meet al of the standards regarding fruit, vegetable and breakfast consumption versus persons who did not meet any of these three standards).

Because psychological distress is known to have a profound association with memory complaints, psychological distress was used in all statistical analyses to adjust for the effects of mood on subjective memory complaints. The questionnaire included ten questions that were based on the depression and anxiety subscales of the Kessler Psychological Distress Scale $(\mathrm{K} 10)^{19}$. Its brevity, strong psychometric properties and ability to discriminate DSMIV psychological distress cases from non-cases make the K10 appropriate for use in health surveys. The K10 consists of ten items that were answered on a five-point Likert scale (where $5=$ "all of the time" and $1=$ "none of the time"). The possible total score ranges from 10 (no distress) to 50 (severe distress). Two groups were formed based on this score: namely, people with low/medium psychological distress and people with high psychological distress (i.e., a score of 30 or more). If responses to one or two questions were missing, imputation based on all participants' average score on that particular question was conducted. Persons with three or more missing values on this questionnaire were excluded from the analyses.

\section{Statistical Analyses}

Multivariate logistic regression analysis was conducted to determine the capability of multimorbidity to predict subjective memory complaints. Next, two in-depth analyses were performed to obtain a more detailed view of the predictive capability of multimorbidity in participants who perceived themselves as forgetful. First, among people who perceived themselves as forgetful, multimorbidity as a prognostic factor for the degree of worrying about these memory complaints was evaluated. Second, among people who perceived 
themselves as forgetful, multimorbidity as a predictor for increased subjective memory complaints perceived during the last year was investigated.

Multimorbidity was conceptualized by a simple disease count. First, simple logistic regression models (unadjusted models), using only multimorbidity as a predictor, were separately conducted for all three outcome measures. Second, three multivariate analyses were conducted, including all confounders. Third, the model was expanded by including the relevant interaction terms. In all three analyses, interaction terms of (multi)morbidity with age and (multi)morbidity with sex were included because the predictive capability of multimorbidity for memory complaints was expected to differ between men and women and between age groups. Only the interaction terms that appeared significant are reported here. The multivariate analyses were conducted with corrections for sex, age, educational level, smoking status, alcohol intake, living arrangements, exercise, eating habits and psychological distress. Categorical confounders were coded into dummy variables. Potential confounders that were not significantly related to memory complaints were excluded from the final analyses (P-values $>0.05$ ). The confounders of gender, age and education always remained in the final analyses, irrespective of significance. A stepwise backward method was employed using the statistical software package SPSS version 17. Only persons with no missing data were included in the analyses. Odds ratios (OR) and 95\% confidence intervals (CI) are reported. P-values of 0.05 or less were considered statistically significant.

\section{RESULTS}

Descriptive characteristics of the 15,188 participants are shown in Table 1 . More than $26 \%$ of the participants considered themselves forgetful, and more than $50 \%$ suffered from multimorbidity.

In the unadjusted analyses, multimorbidity appeared to be a significant predictor of subjective memory complaints (see Table 2). In the interaction analyses, there was a significant two-way interaction between multimorbidity and age, indicating that the predictive capability of multimorbidity for subjective memory complaints was stronger in people aged 55 to 69 (OR: 2.08) than in people aged 70 and over (OR: 1.48) (see Table 3).

The two-way interaction between multimorbidity with three or more diseases present and age was also significant (OR: 3.09 for people aged 55 to 69 and OR: 2.11 for people aged 70 and over). Interactions between morbidity and age and between multimorbidity and sex were not significantly related to the presence of subjective memory complaints. Psychological distress and healthy eating habits both appeared to be significant prognostic factors of subjective memory complaints.

Among participants who perceived themselves as forgetful, suffering from multimorbidity appeared to be a significant predictor of a higher degree of worrying about subjective memory complaints (see Table 2). After adjustment for significant confounders, multimorbidity remained a significant predictor (see Table 3). In interaction analyses, no significant interactions with multimorbidity were detected. Psychological distress was indicative of a higher degree of worrying about subjective memory complaints (see table 5). 
Table 1. Descriptive characteristics of the study population $(\mathrm{N}=15,188)$

\begin{tabular}{lc}
\hline Characteristics & \\
\hline People aged 55 to 69 (\%) & $7556(49.7)$ \\
Gender (\% females) & $8063(33.1)$ \\
Perceived forgetfulness (\% yes) & $3976(26.2)$ \\
Educational level & \\
Low & $3893(25.7)$ \\
Medium & $8194(53.9)$ \\
High & $2047(13.5)$ \\
(Multi)morbidity score diagnosed by a medical doctor (\%) & \\
0 & $4067(26.8)$ \\
1 & $3392(22.3)$ \\
2 & $2502(16.5)$ \\
$\geq 3$ & $5227(34.4)$ \\
Excessive alcohol drinker (\% yes) & $1176(7.7)$ \\
Smoking (\% yes) & $2567(16.9)$ \\
Exercise (\% meet the norm) & $8595(56.6)$ \\
Eating habits (\% meet all 3 norms) & $2296(15.1)$ \\
Living arrangement (\% living alone) & $3882(25.6)$ \\
Psychological distress (\% high risk) & $983(6.5)$ \\
\hline
\end{tabular}

Mean age was 70 (age ranged from 55 to 90 years) Educational level: wow is defined as primary school, lower medium or lower vocational education; medium as secondary school and/or medium level vocational education; high as higher vocational education or university degree. For $6.9 \%$ of individuals this information was missing. Excessive alcohol drinker: $>21$ glasses per week (men) or $>14$ glasses per week (women) Eating habits: meeting all three norms for fruit, vegetable and breakfast consumption.

Table 2. Uncorrected relations between (multi)morbidity and memory complaints, degree of worry about memory complaints and the increase of memory complaints perceived during the last year (odds ratio and $95 \% \mathrm{CI}$ ).

\begin{tabular}{lccc}
\hline & Memory complaints & $\begin{array}{c}\text { Degree of worry about } \\
\text { memory complaints }\end{array}$ & $\begin{array}{c}\text { Memory complaints } \\
\text { during the last year }\end{array}$ \\
\hline (Multi)morbidity & $1.29(1.12-1.47)^{* *}$ & $1.42(0.94-2.17)$ & $1.48(0.87-2.52)$ \\
1 disease & $1.78(1.55-2.05)^{* *}$ & $1.00(0.63-1.59)$ & $1.13(0.64-2.01)$ \\
2 diseases & $3.02(2.68-3.39)^{* *}$ & $2.65(1.87-3.74)^{* *}$ & $2.28(1.46-3.54)^{* *}$ \\
$\geq 3$ diseases &
\end{tabular}

${ }^{*} \mathrm{p}<.05 ;{ }^{* *} \mathrm{p}<.01$ The reference group consisted of people with none of the included medical conditions 


\section{Chapter 2}

Table 3. Corrected relations between (multi)morbidity and memory complaints, degree of worry about memory complaints and the increase in memory complaints perceived during the last year (odds ratio and $95 \% \mathrm{CI}$ corrected for age, sex, education and psychological stress).

\begin{tabular}{lccc}
\hline & Memory complaints & $\begin{array}{c}\text { Degree of worry about } \\
\text { memory complaints }\end{array}$ & $\begin{array}{c}\text { Memory complaints } \\
\text { during last year }\end{array}$ \\
\hline (Multi)morbidity & $1.2(1.05-1.38)^{* *}$ & $1.35(0.88-2.07)$ & $1.32(0.77-2.281)$ \\
1 disease & $1.65(1.43-1.90)^{* *}$ & $0.97(0.61-1.56)$ & $1.04(0.58-1.87)$ \\
2 diseases & $2.34(2.07-2.64)^{* *}$ & $1.93(1.35-2.76)^{* *}$ & $1.33(0.84-2.11)$ \\
$\geq 3$ diseases &
\end{tabular}

${ }^{*} \mathrm{p}<.05 ;{ }^{* *} \mathrm{p}<.01 \mid$ The reference group consisted of people with none of the included medical conditions

Table 4. Relation between (multi)morbidity and memory complaints in the full study sample (corrected odds ratio and $95 \% \mathrm{CI}$ resulting from logistic regression analyses)

\begin{tabular}{lc}
\hline & Memory complaints (yes vs. no) \\
\hline Age & $1.04(1.03-1.04)^{* *}$ \\
Sex & $0.84(0.76-0.91)^{* *}$ \\
Multi)morbidity $^{\mathrm{a}}$ & \\
$\quad 1$ disease & $1.19(1.04-1.37)^{*}$ \\
$\quad 2$ diseases & $1.61(1.39-1.86)^{* *}$ \\
$\quad \geq 3$ diseases & $2.28(2.01-2.58)^{* *}$ \\
Educational level & \\
Medium & $0.87(0.78-0.97)^{* *}$ \\
High & $0.86(0.738-0.99)^{*}$ \\
Excessive alcohol drinker & $0.99(0.84-1.18)$ \\
Smoking & $1.01(0.88-1.15)$ \\
Exercise & $1.02(0.93-1.12)$ \\
Eating habits & $0.68(0.60-0.77)^{* *}$ \\
Living arrangement & $1.10(0.99-1.23)$ \\
Psychological distress & $2.67(2.24-3.17)^{* *}$ \\
Interaction & \\
Age $^{*}$ multimorbidity $(2$ diseases $)$ & $0.71(0.53-0.95)^{*}$ \\
Age $^{*}$ multimorbidity $(>3$ diseases $)$ & $0.68(0.54-0.87)^{* *}$ \\
\hline
\end{tabular}

${ }^{*} \mathrm{p}<.05 ;{ }^{* *} \mathrm{p}<.01 \mid$ Note: $0=$ no memory complaints $1=$ memory complaints; $1=$ male, $2=$ female a reference group consisted of people with none of the included medical conditions $\mid \mathrm{b}$ Reference group consisted of people with low education 
Table 5. Relations between (multi)morbidity and the degree of worry about memory complaints and the increase of memory complaints during the last year among participants who perceived themselves as forgetful (corrected OR and 95\% CI resulting from logistic regression analyses)

\begin{tabular}{|c|c|c|}
\hline & $\begin{array}{c}\text { Degree of worry about memory } \\
\text { complaints } \\
(\mathrm{n}=3,598)\end{array}$ & $\begin{array}{c}\text { Increase memory complaints } \\
\text { during last year } \\
(\mathrm{n}=3,441)\end{array}$ \\
\hline Age & $1.28(1.01-1.62)^{*}$ & $0.66(0.48-0.91)^{*}$ \\
\hline Sex & $1.22(0.98-1.53)$ & $3.13(1.14-8.62)^{*}$ \\
\hline \multicolumn{3}{|l|}{ (Multi)morbidity ${ }^{a}$} \\
\hline 1 disease & $1.35(0.88-2.07)$ & $2.37(0.84-6.65)$ \\
\hline 2 diseases & $0.97(0.61-1.56)$ & $2.72(0.97-7.66)$ \\
\hline$\geq 3$ diseases & $1.93(1.35-2.76)^{* *}$ & $2.96(1.16-8.62)^{*}$ \\
\hline \multicolumn{3}{|l|}{ Educational level $^{\mathrm{b}}$} \\
\hline Medium & $0.79(0.62-1.01)$ & $1.03(0.76-1.40)$ \\
\hline High & $1.05(0.72-1.54)$ & $0.90(0.52-1.56)$ \\
\hline Excessive alcohol drinker & $0.77(0.42-1.40)$ & $1.16(0.96-1.42)$ \\
\hline Smoking & $1.05(0.73-1.52)$ & $0.88(0.56-1.38)$ \\
\hline Exercise & $0.86(0.67-1.13)$ & $0.51(0.38-0.70)^{* *}$ \\
\hline Eating habits & $0.60(0.350-1.02)$ & $0.58(0.32-1.02)$ \\
\hline Living arrangement & $1.20(0.91-1.59)$ & $1.13(0.80-1.60)$ \\
\hline Psychological distress & $4.89(3.82-6.24)^{* *}$ & $5.17(3.81-7.02)^{* *}$ \\
\hline \multicolumn{3}{|l|}{ Interaction $^{\mathrm{a}}$} \\
\hline $\begin{array}{l}\text { Sex * multimorbidity } \\
(2 \text { diseases })\end{array}$ & NS & $0.21(0.06-0.76)^{*}$ \\
\hline $\begin{array}{l}\text { Sex * multimorbidity } \\
\text { (3 diseases) }\end{array}$ & NS & $0.301(0.10-0.88)^{*}$ \\
\hline
\end{tabular}

NS= not significant and therefore omitted $\mid$ Note: $0=$ low degree of memory complaints, $1=$ high degree of memory complaints $\mid 0=$ small increase last year, $1=$ large increase last year $\mid 1=$ male, $2=$ female. ${ }^{*} \mathrm{p}<.05$; ${ }^{* *} \mathrm{p}<.01$ a Reference group consisted of people with none of the included medical conditions $b$ Reference group consisted of people with low education

Among participants who perceived themselves as forgetful, multimorbidity appeared to be a significant predictor for reporting increased memory complaints during the last year (see Table 2). However, when corrected for the presence of depressive symptoms, multimorbidity did not remain a significant predictor of the degree of increase in memory complaints during the last year (see Table 3). There was a significant two-way interaction between multimorbidity and sex, indicating that the predictive capability of multimorbidity (i.e., multimorbidity 2) was higher among men (OR: 2.72) than among women (OR: 1.79) (see Table 5). Moreover, there was a significant two-way interaction between multimorbidity (i.e., multimorbidity 3 or higher) and sex (OR: 2.96 for men and OR: 1.12 for women). Interaction terms between morbidity and sex and between multimorbidity and age were not significantly related to an increase in memory complaints during the last year.

Psychological distress and exercise both appeared to be significant prognostic factors of subjective memory complaints. Living arrangements, alcohol consumption, smoking behavior, and exercise did not significantly contribute to any of the three outcome measures and were therefore removed from all statistical analyses. 


\section{DISCUSSION}

The primary aim of the study was to assess the predictive capability of multimorbidity for subjective memory complaints in community-dwelling older adults aged 55 and over living independently in Limburg, the Netherlands. The results indicate that multimorbidity can indeed act as a predictive factor for subjective memory complaints. The predictive capability of multimorbidity for self-perceived forgetfulness was higher among people aged 55 to 69 than among people aged 70 and above.

In participants who suffered from three or more co-occurring chronic diseases and who perceived themselves as forgetful, multimorbidity was a significant predictor of the degree of worrying about subjective memory complaints. This finding indicates that multimorbidity was indeed indicative of a higher degree of worrying about one's selfperceived forgetfulness.

Among participants who perceived themselves as forgetful, multimorbidity appeared to be a significant predictor of the degree of increase in memory complaints during the last year. However, when corrected for psychological distress, this association disappeared. Men who perceived themselves as forgetful and who suffered from multimorbidity reported a larger increase of their memory problems during the last year than did women who perceived themselves as forgetful and suffered from multimorbidity.

This is the first study that aimed to assess the predictive capability of multimorbidity for subjective memory complaints. Several explanations for the predictive capability of multimorbidity could be brought forward. First, in patients suffering from multimorbidity, self-reported memory complaints may reflect a real decline in objective memory performance, which may be mediated by poor health status. Unexpected common pathogenetic mechanisms, such as autoimmune responses, genetics, inflammation and/or immunological mechanisms, might contribute to this association. Some studies did find an association between the total number of morbid conditions and poor performance on memory tests ${ }^{13,20}$. Second, a patient's knowledge that he or she is suffering from multiple medical conditions may change his or her subjective assessment of other health-related problems. People who are suffering from multimorbidity may tend to overestimate the risk of acquiring more health-related conditions, which may, among other consequences, result in more complaints and a higher degree of worrying about their self-perceived forgetfulness ${ }^{21}$. Third, it could be argued that memory complaints are completely secondary to psychological distress, which may influence a person's evaluation of their memory functions. However, after controlling for psychological distress, multimorbidity remained a significant predictor of subjective memory complaints.

The question remains of why the predictive capability of multimorbidity for subjective memory complaints is stronger among people aged 55 to 69 than among people aged 69 and over. Younger people may still be active in professional activities and managing a workload and may therefore perceive more direct hindrance due to their forgetfulness in daily life. Hence, older individuals might evaluate their memory complaints as being more common than do younger individuals who are confronted with age-related memory complaints at an earlier stage in life $\mathrm{e}^{22}$. 
In short, attention should be paid to the physical as well as the cognitive consequences of multimorbidity. Early identification and monitoring of people with multimorbidity could provide opportunities for controlling the negative health-related consequences of multimorbidity, including subjective memory complaints. Older patients with an increased probability of subjective memory problems, possibly mediated by a poor morbidity status, may be identified by routinely inquiring about memory complaints. Early inquiry is especially important in those patients suffering from three or more diseases and those in whom memory complaints are indeed a consequence of objective memory decline. These patients can then be monitored appropriately, including by a general practitioner ${ }^{3}$. If objective memory performance is found to be normal, patients need to be reassured that their worry is unfounded and that their forgetfulness can be ascribed to normal forgetfulness, which tends to increase with age. Psychosocial interventions aimed at reducing psychological distress related to these complaints may be beneficial for people with subjective memory complaints ${ }^{23}$.

Another finding of the current study was that participants who perceived themselves as forgetful and who suffered from high psychological distress worried more about their reported memory problems and reported a larger increase in these problems during the last year as compared to persons with lower psychological distress. These findings are in line with previous research that claims that anxiety and psychosocial stress may affect both patients' perception of their own memory performance and their objective memory performance $^{7,24}$. Because self-observations of memory decline may also lead to depressive feelings, the relationship between depressive feelings and subjective memory complaints may be a reciprocal one ${ }^{25}$.

Two other intriguing findings were present in this study. First, persons who met the norm for healthy eating (i.e., the norm for vegetables, fruit or breakfast consumption) had a lower risk of reporting memory complaints. Moreover, people who met the standard for exercise had a lower risk of reporting increased memory complaints during the last year. This result is in accordance with previous research indicating that a healthy eating pattern and sufficient exercise are related to a variety of profitable health-related outcomes, including a lower risk for diabetes mellitus type $\mathrm{II}^{26}$ and cancer ${ }^{27}$.

One of the strong points of this study was its use of data based on a large number of participants aged 55 years and over living in the community. Consequently, the results are representative for community-dwelling older individuals and are therefore more generalisable than results of studies conducted in more homogeneous populations ${ }^{28}$. Furthermore, several relevant health-related factors have been used to control for possible confounding.

Despite these strengths, our findings must be interpreted in light of some possible limitations. First, multimorbidity was conceptualized based on participants' self-reports, which could have compromised the validity of these data. Although self-reported morbidity status has been shown to correlate reasonably well with medical records, the oldest old (i.e., people aged 90 and over) tend to under-report certain diagnosed medical conditions ${ }^{29}$. Secondly, the present study did not control for the presence of Mild Cognitive Disorder or dementia in participants. Because participants were required to be cognitively intact to fill 


\section{Chapter 2}

in the questionnaire, the percentage of participants with sincere objective memory problems is expected to be small. Third, the present study is of a cross-sectional nature, which means that no conclusions can be drawn regarding the longitudinal effects of multimorbidity on subjective memory complaints. Because memory complaints due to health status are likely to develop over the course of many years, ideally the underlying process should be investigated longitudinally. Hence, a well-conducted longitudinal study with adequate control for confounding factors is warranted to evaluate the effects of multimorbidity on the evolution of subjective memory complaints. Future research should also explore the role of multimorbidity as assessed by using a more objective approach, such as a general practice database that incorporates all medical diseases diagnosed by a general practitioner. It might be argued that the predictive capability of multimorbidity as assessed by a more objective approach is less strong than that found in the present study.

In conclusion, our findings indicate that multimorbidity and subjective memory complaints are both common in a population of older adults and appear to be related. Taking into account previous research that suggested that subjective memory complaints may be related to future memory decline, the present results may have implications for clinical practice in an aging population. To protect people's quality of life and to save a considerable amount in health care expenditures, adequate early intervention programs to ameliorate the negative health-related consequences of multimorbidity are called for. 


\section{REFERENCES}

1. R.W. Ponds, K.J. Commissaris, and J. Jolles. Prevalence and covariates of subjective forgetfulness in a normal population in The Netherlands. Int. J. Aging Hum. Dev., 1997;45(3):207-221.

2. C.J. Commissaris, J. Jolles, F.R. Verhey, R.W. Ponds, V. Damoiseaux, and G.J. Kok. Forgetful or demented? Who worries and why? Tijdschrift voor Gerontologie en Geriatrie, 1993;24(4):144-149.

3. S. Iliffe and L. Pealing. Subjective memory problems. BMJ, 2010;340:1425.

4. C. Jonker, M.G. Dik, G.J. van Kamp, and D.J. Deeg. Apolipoprotein E4 and memory decline in the elderly. Tijdschrift voor Gerontologie en Geriatrie 2000;31(5):198-202.

5. M.I. Geerlings, C. Jonker, L.M. Bouter, H.J. Ader, and B. Schmand. Association between memory complaints and incident Alzheimer's disease in elderly people with normal baseline cognition. Am. J. Psychiatry, 1999;156(4):531-537.

6. J.M. Berry and R.L. West. Cognitive Self-efficacy in Relation to Personal Mastery and Goal Setting across the Life Span. International Journal of Behavioral Development, 1993;16(2):351-379.

7. M.E. Mol, M.P. van Boxtel, D. Willems, and J. Jolles. Do subjective memory complaints predict cognitive dysfunction over time? A six-year follow-up of the Maastricht Aging Study. Int. J. Geriatr. Psychiatry, 2006;21(5):432-441.

8. C. Elfgren, L. Gustafson, S. Vestberg, and U. Passant. Subjective memory complaints, neuropsychological performance and psychiatric variables in memory clinic attendees: A 3-year follow-up study. Arch. Gerontol. Geriatr., 2010;51(3):110-114.

9. T. Hanninen, K.J. Reinikainen, E.L. Helkala, K. Koivisto, L. Mykkanen, M. Laakso, K. Pyorala, and P.J. Riekkinen. Subjective memory complaints and personality traits in normal elderly subjects. Journal of the American Geriatric Society, 1994;42(1):1-4.

10. M. Mol, M. Carpay, I. Ramakers, N. Rozendaal, F. Verhey, and J. Jolles. The effect of perceived forgetfulness on quality of life in older adults; a qualitative review. international J. Geriatr. Psychiatry, 2007;22(5):393-400.

11. F.B. Waldorff, V. Siersma, and G. Waldemar. Association between subjective memory complaints and health care utilisation: a three-year follow up. BMC Geriatr., 2009;23(9):43.

12. M. van den Akker, F. Buntinx, and J. Knottnerus. Comorbidity or multimorbidity: What's in a name. A review of literature. Eur. J. Gen. Pract., 1996;2:65-70.

13. H.C. Comijs, D.M. Kriegsman, M.G. Dik, D.J. Deeg, C. Jonker, and W.A. Stalman. Somatic chronic diseases and 6-year change in cognitive functioning among older persons. Arch. Gerontol. Geriatr., 2009;48(2):191-196.

14. M.P. van Boxtel, F. Buntinx, P.J. Houx, J.F. Metsemakers, A. Knottnerus, and J. Jolles. The relation between morbidity and cognitive performance in a normal aging population. J. Gerontol. A. Biol. Sci. Med. Sci., 1998;53(2):147-154.

15. P.C. Elwood, J. Pickering, A. Bayer, and J.E. Gallacher. Vascular disease and cognitive function in older men in the Caerphilly cohort. Age Ageing, 2002;31(1):43-48.

16. C. Vogeli, A.E. Shields, T.A. Lee, T.B. Gibson, W.D. Marder, K.B. Weiss, and D. Blumenthal. Multiple chronic conditions: prevalence, health consequences, and implications for quality, care management, and costs. J. Gen. Intern. Med., 2007;22 (3):391-395.

17. J.M. Guralnik. Assessing the impact of comorbidity in the older population. Ann. Epidemiol., 1996;6(5):376-380.

18. M. Douwes and V.H. Hildebrandt. Vragen naar de mate van lichamelijke activiteit. Geneeskunde en Sport, 2000;33(1):9-16.

19. T. Donker, H. Comijs, P. Cuijpers, B. Terluin, W. Nolen, F. Zitman, and B. Penninx. The validity of the Dutch K10 and extended K10 screening scales for depressive and anxiety disorders. Psychiatry Res., 2010;176(1):45-50.

20. H.T. Nguyen, M.K. Evans, and A.B. Zonderman. Influence of medical conditions on executive and memory functions in low socioeconomic status African Americans. Archives of Clinical Neuropsychology, 2007;22(6):689-698. 


\section{Chapter 2}

21. J.M. Hodek, A.K. Ruhe, and W. Greiner. Relationship Between Health-Related Quality of Life and Multimorbidity. Gesundheitswesen, 2010;72:455-465.

22. M.E. Mol, R.A. Ruiter, F.R. Verhey, J. Dijkstra, and J. Jolles. A study into the psychosocial determinants of perceived forgetfulness: implications for future interventions. Aging and Mental Health, 2008;12(2):167-176.

23. C.S. Hurt, A. Burns, R.G. Brown, and C. Barrowclough. Perceptions of subjective memory complaint in older adults: the Illness Perception Questionnaire - Memory (IPQ-M). Int. Psychogeriatr., 2010;22(5):750-760.

24. A.F. Jorm, P. Butterworth, K.J. Anstey, H. Christensen, S. Easteal, J. Maller, K.A. Mather, R.I. Turakulov, W. Wen, and P. Sachdev. Memory complaints in a community sample aged 60-64 years: associations with cognitive functioning, psychiatric symptoms, medical conditions, APOE genotype, hippocampus and amygdala volumes, and white-matter hyperintensities. Psychol. Med., 2004;34(8):1495-1506.

25. B. Schmand, C. Jonker, M.I. Geerlings, and J. Lindeboom. Subjective memory complaints in the elderly: depressive symptoms and future dementia. Br. J. Psychiatry, 1997;171:373-376.

26. J. Ma, A.C. King, S.R. Wilson, L. Xiao, and R.S. Stafford. Evaluation of lifestyle interventions to treat elevated cardiometabolic risk in primary care (E-LITE): a randomized controlled trial. BMC Fam. Pract., 2009;10:71.

27. C.E. Mosher, R. Sloane, M.C. Morey, D.C. Snyder, H.J. Cohen, P.E. Miller, and W. DemarkWahnefried. Associations between lifestyle factors and quality of life among older long-term breast, prostate, and colorectal cancer survivors. Cancer, 2009;115(17):4001-4009.

28. D. Lahr, T. Beblo, and W. Hartje. Cognitive performance and subjective complaints before and after remission of major depression. Cognitive Neuropsychiatry, 2007;12(1):25-45.

29. S. Goebeler, M. Jylha, and A. Hervonen. Self-reported medical history and self-rated health at age 90. Agreement with medical records. Aging Clinical and Experimental Research, 2007;19(3):213-219. 


\section{CHAPTER 3}

Influence of multimorbidity on cognitive decline in a normal aging population: a 12 year follow-up in the Maastricht Aging Study

Published as (in shortened version):

S Aarts, M. van den Akker, F.E.S. Tan, F.R.J Verhey, J.F.M. Metsemakers and M.P.J. van Boxtel. International Journal of Geriatric Psychiatry, 2011;26(10):1046-1053. 


\title{
Chapter 3
}

\begin{abstract}
Background

Multimorbidity has risen considerably because of the increase in longevity and the rapidly growing number of older individuals. Today, only little is known about the influence of multimorbidity on cognitive decline in a normal healthy aging population. The primary aim of the present study was to investigate the effect of multimorbidity on cognitive functioning over a 12 year period in an adult population with a large age range.
\end{abstract}

\section{Methods}

Data were collected as part of the Maastricht Aging Study (MAAS), a prospective study into the determinants of cognitive aging. Eligible MAAS participants $(\mathrm{N}=1.763)$, aged 2481 years, were recruited from the Registration Network Family Practices which enabled the use of medical records. The association between 96 chronic diseases, grouped into 23 clusters, and cognitive functioning on baseline and at 6 and 12 years of follow-up were analyzed. Cognitive performance was measured in two domains: verbal memory and psychomotor speed. A multilevel statistical analysis, a method that respects the hierarchical data structure, was used.

\section{Results}

Multiple clusters of chronic medical conditions were associated with cognitive decline during a 12-year follow-up period in a healthy adult population. Apart from the disease combination malignancies and movement disorders multimorbidity appeared not to significantly affect cognition.

\section{Conclusions}

The current results indicate that morbidity status adversely affects cognitive functioning. However, the effects of morbidity and multimorbidity status on cognitive decline appear to be small in a normal healthy aging population. 


\section{INTRODUCTION}

In today's society, the occurrence of (multiple) chronic medical conditions has risen considerably because of the increase in longevity and the rapidly growing number of older individuals. Various prevalence estimates of multimorbidity, a term used to describe the cooccurrence of two or more chronic conditions within one person ${ }^{1}$, have been reported, ranging from $30 \%{ }^{2}, 62 \%^{3}$ to over $68 \%^{4}$ of the population under study. (Multi)morbidity is associated with several poor outcomes such as: activity limitations ${ }^{5}$, decreased health related quality of life ${ }^{6}$ and impaired cognitive functioning ${ }^{7}$. Several studies have suggested that impaired health status may enhance cognitive decline in normal aging but may also enhance pathological cognitive aging ${ }^{7,8}$.

Various single disease entities have already been related to a decrease in cognitive performance. Evidence to date suggests that disorders such as diabetes mellitus type I and $\mathrm{II}^{9}$, cardiovascular diseases ${ }^{10}$, stroke $^{11}$ and peripheral atherosclerosis ${ }^{12}$ may all be associated with decreased cognitive functioning. In addition, several diseases are also known to enhance the development of dementia ${ }^{8}$. For example, after a ischemic stroke, dementia develops in approximately $25 \%$ of patients ${ }^{13}$. Moreover, another study concluded that the attributable risk of diabetes for dementia is 8.8 percent $^{14}$.

Several studies focused on the relationship between a specific chronic medical condition and cognition, which most often implies the exclusion of persons with comorbid diseases ${ }^{12}$. Since research suggests that up to 68 percent of persons in a hospital setting suffer from multimorbidity ${ }^{15}$, studies limited to persons with only one single disease entity may therefore be less representative. Moreover, it has been suggested that in a geriatric population cumulative illness is associated with more enhanced cognitive decline compared to elderly with one single diseases ${ }^{15}$. The majority of the studies that focussed on the relationship between morbidity and cognition have been cross-sectional9,15. However, since cognitive decrements due to health status are likely to develop over the course of many years, ideally the underlying process should be investigated longitudinally. In most cases, longitudinal studies included only a small number of participants ${ }^{7}$ or were limited to specific population samples, such as older persons ${ }^{12}$ or patients admitted for clinical rehabilitation ${ }^{12,15}$. Therefore, it is unclear how multimorbidity affects cognitive functioning in a normal aging adult population. Furthermore, since most studies did not include younger participants, it is unclear to what extent these people experience cognitive decline which may be mediated by their health status.

So far, research involving the association between morbidity and cognition was mainly restricted to evaluate only a few chronic medical conditions. In a longitudinal study with six years of follow-up, the influence of seven somatic chronic diseases on changes in cognitive functioning was studied in persons aged 62 to 85 years $^{12}$. However, in order to specify the differential effects of various medical conditions on cognitive functioning, it is necessary to evaluate a greater variety of medical conditions. Furthermore, most studies reporting on the association between morbidity and cognition were based on self-reported morbidity status ${ }^{12}$. As the agreement between self-reports as medical records tends to weaken in older age groups ${ }^{16}$, this is highly problematic when studying the effects of 
multimorbidity in older persons. Moreover, in patients suffering from multiple medical conditions, the agreement between self-reports and medical records also tends to be lower ${ }^{17}$. Hence, medical records from general practitioners (GPs) may provide more valid data on prevalent morbidity, especially in countries such as the Netherlands, were GPs are the gatekeepers to other health care facilities, thus having a comprehensive view of their patient's health status.

The primary aim of the present study was to investigate the effect of (multi)morbidity on cognitive functioning, measured by established neurocognitive tests, in an adult population with a wide age range. A longitudinal study was conducted over a 12-year period, in order to evaluate the effect of 96 chronic medical conditions, grouped into clusters, on cognitive functioning.

\section{METHODS}

The present study was conducted on data gathered in two projects: The Registration Network Family Practices (RNH) and the Maastricht Aging Study (MAAS). MAAS is a prospective study of determinants of usual cognitive aging ${ }^{18}$. Participants of MAAS were recruited from the Registration Network Family Practices (Registratie Netwerk Huisartspraktijken, RNH), which contains demographic and health information of over 120.000 patients inhabited in the South of Limburg, the Netherlands ${ }^{19}$.

\section{Maastricht Aging Study (MAAS)}

In 1992, 10.396 people were recruited from the RNH database of whom 4.490 were willing to participate $(43.2 \%), 3.531$ refused participation (34\%), and $2.375 \mathrm{did}$ not respond (22.8\%). Since MAAS' primary aim was to investigate healthy aging, participants were excluded from baseline participation when medical records from the RNH database contained the following conditions known to interfere with cognition: history of coma, cerebrovasculair disorder, tumour of the nervous system, congenital malformation of the nervous system, multiple sclerosis, Parkinsonism, epilepsy, dementia, organic psychosis, schizophrenia, affective psychosis or mental retardation. The 4,490 eligible participants were screened by telephone for medical conditions not documented in the RNH database (history of transient ischemic attack, brain surgery, haemodialysis for renal failure, electroconvulsive therapy or daily psychotropic drug use). Of the remaining 4,189 participants (301 were excluded based on the telephone screening), 1,823 (aged 24 to 81 years at baseline) were randomly selected, stratified for age (12 discrete groups between 24 and 81 years of age at baseline), sex and occupational achievement (2 levels).

\section{Registration Network Family Practices (RNH)}

The morbidity status of all MAAS participants at baseline, 6- and 12-year follow-up was retrieved from the RNH database. The RNH is a continuously updated database, which contains the medical records of patients from 21 family practices in which 65 general practitioners (GPs) working in the south of the Netherlands are participating. This

database includes all relevant current and past health problems. A health problem was 
defined as 'anything that has required, does or may require health care management and has affected or could significantly affect a person's physical or emotional well-being ${ }^{20}$. A diagnosis is made by the GP or by a medical specialist to whom a patient may be referred. Especially in complex medical conditions, registration is often based on a specialist diagnosis reported to the GP. The RNH database contains health problems which are only coded by the GP when they are permanent (no recovery expected), chronic (duration longer than 6 months) or recurrent (more than three recurrences within 6 months), or when they have lasting consequences for the functional status or prognosis of the patient. Problems are coded in a standardized fashion, according to the International Classification of Primary Care (ICPC) $)^{21}$, using the criteria of the International Classification of health in Primary Care (ICHPPC-2) and other more current guidelines of the Dutch College of GP's. The database also contains background information about the patient's sex, date of birth, marital status, living arrangement and level of education. Membership of the RNH registry ends by migration or death. All patients included in the RNH database have been informed about the potential anonymous use of their health information. If a patient does not agree with the use of his/her health information, the inclusion of this patient in the $\mathrm{RNH}$ database can be stopped. The quality of the data is ascertained by ample instruction and training sessions, regular regional consensus groups, quality control audits, an online thesaurus available during data-entry and systematic control for erroneous or missing entries. Sociodemographical characteristics of samples in other studies which made use of the RNH database have been shown to be comparable to the Dutch population ${ }^{22}$.

\section{Outcome Measures}

Two representative neuropsychological tests were used to assess the cognitive domains of memory and processing speed at all three measurements points.

\section{Visual Verbal Learning test}

The Visual Verbal Learning test (VVLT) is the Dutch version of the Rey VVLT, which measures the ability to learn new verbal information and retrieve information from memory ${ }^{23}$. Fifteen monosyllabic words were visually presented one at a time on five consecutive trials. Participants were asked to recall as many words as possible, with no restriction concerning the order of recall (immediate recall). After a 20 minutes delay, participants were again asked to recall as many correct words as possible (delayed recall). This was followed immediately by a recognition test, involving the previous seen fifteen words intermixed with fifteen new words $s^{23}$. The latter score (ranging from 0 to 15) was used as outcome measure. Parallel versions of the memory tasks were used for each assessment.

\section{Letter Digit Substitution Task}

The Letter Digit Substitution Task (LDST) is used to measure complex information processing under time pressure ${ }^{24,25}$. A sheet with letters, each matched to its own number (1 to 9) was presented to the participant. Participants were asked to match as much letters and numbers as possible in 90 seconds, by filling in the right numbers belonging to the right letters. The total number of filled in numbers was used as outcome measure. 


\section{Independent Variables}

Based on either previously conducted studies or from clinical experience, 96 chronic medical conditions with (possible) high prevalence and/or potential to impact brain functioning were included in the analyses, namely: malignancies, movement disorders, chronic respiratory diseases, cardiovascular diseases (heart failure, ischemic diseases, arrhythmias and cerebrovascular diseases), endocrine diseases, neurological disturbances and psychological disorders. The medical conditions on which exclusion for MAAS participation was based were also included in order to monitor possible occurrences after baseline.

Beforehand, single morbidity codes were combined into clusters of morbidities by three experts (MvdA, MvB, JM), including 2 medical doctors ( $\mathrm{vvB}, \mathrm{JM})$, to limit the number of independent variables. Some morbidity codes were not clustered because they were highly prevalent and/or because their effect on cognition has been well documented (diabetes mellitus, dementia and Parkinsonism). A list of all ICPC codes and the clustering of these ICPC codes is presented in Appendix I. Note that one person can belong to two or more clusters when diagnosed with multiple medical conditions. Moreover, a person can be diagnosed with more than one disease in one cluster. Instead of coding each cluster as either being present or absent in a participant, time in years since diagnosis was incorporated in order to take disease duration into account. If a person was diagnosed with more than one disease within one cluster, the cluster was coded with the first date of a diagnosis. The reference group consisted of all participants without any of the 96 diseases. The overall morbidity status of all MAAS participants was assessed by looking at the (present and past) medical records retrieved from the Registration Network Family Practices providing info at baseline and at 6 and 12 year follow-up.

\section{Possible Confounders}

Potential confounders were taken into account: gender, age, education level measured on a 8 -point ordinal scale (ranging from primary education to university degree) ${ }^{26}$, smoking behaviour at baseline (yes or no), alcohol intake at baseline (number of alcoholic units consumed per week) and living arrangements in three categories (living with family, living alone, living in a home for the elderly). Self-reported depression as assessed by the SCL-90 was inserted as a possible confounding factor ${ }^{27}$.

Total morbidity score (in 6 categories, ranging from no disease present to 6 or more diseases present) was assessed by calculating the total number of chronic diseases per individual in the RNH database. Total cluster score (in 6 categories, ranging from no cluster of diseases present to 6 or more clusters present) was assessed by looking at the total number of clusters in which patients had at least one disease.

\section{Statistical Analyses}

As the independent variables (20 clusters and 3 separate chronic medical conditions) and the outcome measures (cognitive functioning at two neuropsychological tests) were measured three times during 12 years, data were analyzed with Linear Mixed Models. This 
procedure respects the hierarchical structure of longitudinal data ${ }^{28}$. Since measurements within subjects of this study are more alike than measurements between subjects, there is correlation between the repeated measurements. This within-subjects variation that accounts for changes within each subject through repeated measurements in time and the between-subjects variation accounting for differences between the subjects performance, can be established by a multilevel approach ${ }^{28}$. Moreover, in a multilevel approach, participants with missing observations are retained in the analysis.

The missing observations are supposed to be Missing At Random. The interpretation of the regression parameters obtained in a multilevel approach is comparable to that of the (one-level) multiple linear regression. As previously stated the dependent variables were measured three times during 12 years (baseline, 6 year follow-up and 12 year follow-up). At baseline, 6 and 12 years follow-up the independent variables (i.e. medical status) were retrieved from the RNH database. However, since reporting the results of the 6 year followup will decrease the comprehension of the present study, only the results at 12 year followup will be reported.

The multilevel models included fixed terms for follow-up (time of measurement), age, gender, education, smoking behaviour, alcohol intake, living arrangements, total morbidity score and total cluster score. Total morbidity score and total cluster score were always tested in separate models. A covariate was included in the model if it significantly influenced the cognitive domain under study $(\mathrm{p}<0.05)$. In order to limit the number of variables in a multilevel model, each cluster (and the 3 separate medical conditions) was evaluated separately using a univariate analysis, resulting in 23 analyses for each cognitive domain.

The effect of multimorbidity on cognitive function was investigated by studying cluster interactions. Seven clusters of chronic diseases were selected, based on prevalence and their significant effect on cognition. First, these seven clusters were evaluated using a multivariate analysis using a backward-method. Next, clusters that appeared significant in this multivariate analysis were used in order to analyze two-way interactions, in order to evaluate the effects of multimorbidity on cognition. All analyses were conducted with the SPSS statistical software package version 16.0 for Windows (SPSS Inc., Chicago, IL, USA). P-values of 0.05 or less were considered statistically significant.

\section{RESULTS}

Descriptive characteristics of the study sample are reported in Table 1 . The data set contained 4196 measurements among 1763 participants. The sample size differed between the three measurements which is due to drop out (i.e. migration, death). At baseline, the average age was 55.4 years with slightly more women (50.4) than men. Multimorbidity was present in $55.9 \%$ of the participants.

Higher education was associated with a significant better performance of memory $(\mathrm{P}<.001)$ and processing speed. Higher age negatively influenced memory and processing speed $(\mathrm{P}<.001)$ and women scored significantly higher than men in both cognitive domains (respectively, $\mathrm{P}<.001$ for memory and $\mathrm{P}<.002$ for processing speed). Depressive complaints, as measured with the Symptom checklist, were associated with impaired memory $(\mathrm{P}=.013)$ and speed $(\mathrm{P}<.001)$. Smoking and alcohol consumption, living arrangements, total 
morbidity score and total cluster score appeared not to affect cognition significantly, and were therefore dropped from all the analyses.

Multiple clusters appeared to be significantly related to cognition (Table 2). Malignancies were related to a lower performance on both cognitive measures as was the ischemic diseases cluster and arrhythmias/heart failure. A lower performance on both cognitive measures was also found among persons with asthma/Chronic Obstructive Pulmonary Disease (COPD)/bronchitis and among persons with gastro-intestinal diseases. Peptic ulcers, diseases of the ear, other cardiovascular diseases, movement disorders, mood disorders, other mental disorders, endocrine diseases or diseases of the urinary tract significantly decreased performance on the processing speed task but not on the verbal memory task. In contrast, Parkinsonism and eczema/psoriasis/chronic skin ulcer was negatively associated with verbal memory, but not with processing speed. Migraine and headache, other diseases of the nervous system and other chronic respiratory diseases were not associated with neither of the two cognitive functions.

The following seven clusters were selected in order to investigate the influence of cluster combinations (i.e. multimorbidity) on cognitive decline: cerebrovasculair diseases, cardiac diseases, malignancies, movement disorders, asthma/COPD/ bronchitis, endocrine diseases and diabetes mellitus. Since the medical conditions in the cluster ischemic diseases and in the cluster other cardiovascular diseases are rather comparable these two clusters were regrouped into one cardiac disease cluster. Consequently, the prevalence of the cardiac cluster was larger than the prevalence of the two individual clusters which resulted in a more robust cardiac cluster.

In the multivariate analyses only the cardiac cluster and malignancies were associated with a significant lower verbal memory performance (see Table 3). Cerebrovascular diseases, cardiac diseases, malignancies and movement disorders were all significantly related to a decrease in processing speed. Hence, these four clusters, namely cerebrovasculair diseases, malignancies, movement disorders and cardiac disease, were selected in order to evaluate the effects of combinations of clusters, and thus of multimorbidity, on cognition in univariate models.

Since only two disease clusters were related to verbal memory in the multivariate model, only one cluster combination, cardiac diseases and malignancies, was evaluated for verbal memory in a univariate model. The interaction of these two disease clusters appeared not to be significantly associated with cognitive decline. Since four disease clusters appeared to significantly affect processing speed in the multivariate model, six cluster combinations were separately investigated in univariate analyses. As displayed in Table 4, only the cluster combination malignancies and movement disorders was related to a faster decline in processing speed. 
Table 1. Descriptive characteristics of the study population $(\mathrm{N}=1763)$

\begin{tabular}{|c|c|}
\hline Characteristics (baseline) & \\
\hline \multicolumn{2}{|l|}{ Follow-up (\# of participants) } \\
\hline Baseline & 1,763 \\
\hline 6 year & 1,310 \\
\hline 12 year & 1,123 \\
\hline Mean age in years $(\mathrm{SD})^{\mathrm{a}}$ & $55.4(15.9)$ \\
\hline Gender (\% females) & $886(50.3)$ \\
\hline \multicolumn{2}{|l|}{ Total morbidity score (\%) } \\
\hline 0 & $404(22.9)$ \\
\hline 1 & $374(21.2)$ \\
\hline $2 \geq$ & $985(55.9)$ \\
\hline \multicolumn{2}{|l|}{ Total cluster score (\%) } \\
\hline 0 & $404(22.9)$ \\
\hline 1 & $427(24.2)$ \\
\hline $2 \geq$ & $932(52.9)$ \\
\hline \multicolumn{2}{|l|}{ Days per week of alcohol intake $(\%)^{b}$} \\
\hline 1 & $467(26.5)$ \\
\hline 2 & $543(30.8)$ \\
\hline 3 & $180(10.2)$ \\
\hline 4 & $78(4.4)$ \\
\hline $5-7$ & $172(9.8)$ \\
\hline \multicolumn{2}{|l|}{ Educational Level ${ }^{\mathrm{c}}$} \\
\hline Low $(\%)$ & $650(36.9)$ \\
\hline Medium (\%) & $712(40.4)$ \\
\hline High (\%) & $399(22.6)$ \\
\hline \multicolumn{2}{|l|}{ Living arrangement } \\
\hline Living with family (\%) & $1490(84.5)$ \\
\hline Living alone (\%) & $235(13.3)$ \\
\hline Home for the elderly/commune (\%) & $8(0.5)$ \\
\hline Unknown/others & $30(1.7)$ \\
\hline SCL-90 Depressive symptom score (SD) ${ }^{\mathrm{d}}$ & $23.1(8.6)$ \\
\hline Smoking (\% NO) & $1250(70.9)$ \\
\hline
\end{tabular}




\section{Chapter 3}

Table 2. Regression coefficients describing the relation between morbidity clusters and cognitive function (corrected for age, sex, education and depressive complaints)

\begin{tabular}{|c|c|c|c|}
\hline & Verbal memory & Processing speed & $\mathrm{N}$ \\
\hline All malignancies & $-0.047^{* *}$ & $-0.341^{* *}$ & 135 \\
\hline Peptic ulcers & -0.233 & $-0.210^{* *}$ & 35 \\
\hline Other gastro-intestinal diseases & $-0.037^{*}$ & $-0.226^{* *}$ & 94 \\
\hline Diseases of the eye & $-0.067^{*}$ & $-0.330^{* *}$ & 34 \\
\hline Diseases of the ear & -0.024 & $-0.123^{*}$ & 65 \\
\hline Ischemic diseases & $-0.061^{* *}$ & $-0.370^{* *}$ & 130 \\
\hline Pulmonary embolism and phlebitis & -0.036 & $-0.246^{* *}$ & 43 \\
\hline Cerebrovascular diseases & $-0.074^{*}$ & $-0.467^{* *}$ & 66 \\
\hline Arrhythmias \& heart failure & $-0.052^{* *}$ & $-0.261^{* *}$ & 108 \\
\hline Other cardiovascular disease & -0.054 & $-0.227^{*}$ & 96 \\
\hline Movement disorders & -0.017 & $-0.087^{* *}$ & 318 \\
\hline Parkinsonism & $-0.536^{*}$ & -0.698 & 5 \\
\hline Migraine \& Headache & 0.004 & -0.065 & 41 \\
\hline Other diseases of nervous system & -0.021 & -0.078 & 12 \\
\hline Mood disorders & -0.220 & $-0.234^{* *}$ & 57 \\
\hline Alzheimer & $-6.220^{* *}$ & $-7.021^{* *}$ & 5 \\
\hline Other mental disorders & -0.005 & $-0.144^{* *}$ & 53 \\
\hline Asthma, COPD and bronchitis & $-0.020^{*}$ & $-0.078^{* *}$ & 114 \\
\hline Other chronic respiratory diseases & -0.010 & -0.052 & 125 \\
\hline Eczema, psoriasis chronic skin ulcer & $-0.028^{* *}$ & -0.065 & 82 \\
\hline Endocrine diseases & -0.018 & $-0.160^{* *}$ & 169 \\
\hline Diabetes mellitus (Type I and II) & $-0.052^{* *}$ & $-0.341^{* *}$ & 101 \\
\hline Diseases of the urinary tract & -0.230 & $-0.117^{*}$ & 44 \\
\hline
\end{tabular}

${ }^{*} \mathrm{p} \leq 0.05^{* *} \mathrm{p} \leq 0.01 \mathbb{N}$ refers to the number of participants at 12 years follow-up

Table 3. Regression coefficients of clusters in multivariate analyses (corrected for age, sex, education and depressive complaints)

\begin{tabular}{lccc}
\hline & $\begin{array}{c}\text { Verbal } \\
\text { memory }\end{array}$ & $\begin{array}{c}\text { Processing } \\
\text { speed }\end{array}$ & $\mathrm{N}$ \\
\hline Cerebrovascular diseases & $\mathrm{NS}$ & $-0.262^{* *}$ & 66 \\
Cardiac diseases & $-0.047^{* *}$ & $-0.183^{* *}$ & 180 \\
Malignancies & $-0.038^{* *}$ & $-0.213^{* *}$ & 135 \\
Movement disorders & $\mathrm{NS}$ & $-0.062^{*}$ & 318 \\
Asthma/COPD/bronchitis & $\mathrm{NS}$ & $\mathrm{NS}$ & 114 \\
Endocrine diseases & $\mathrm{NS}$ & $\mathrm{NS}$ & 169 \\
Diabetes Mellitus & $\mathrm{NS}$ & $\mathrm{NS}$ & 101 \\
\hline
\end{tabular}

${ }^{*} \mathrm{p} \leq 0.05^{* *} \mathrm{p} \leq 0.01 \quad \mathrm{NS}=$ not significant $\mathbb{N}$ refers to the umber of participants at 12 years follow-up 
Table 4. Regression coefficients of cluster combinations in univariate analyses (corrected for age, sex, education and depressive complaints)

\begin{tabular}{|c|c|c|c|}
\hline & $\begin{array}{l}\text { Verbal } \\
\text { memory }\end{array}$ & $\begin{array}{c}\text { Processing } \\
\text { speed }\end{array}$ & $\mathrm{N}$ \\
\hline Cardiac diseases * malignancies & NS & NS & 40 \\
\hline Malignancies * movement disorders & NA & $-0.012^{*}$ & 53 \\
\hline Malignancies * cerebrovascular diseases & NA & NS & 16 \\
\hline Cerebrovascular diseases ${ }^{*}$ movement disorders & NA & NS & 32 \\
\hline Cerebrovascular diseases ${ }^{*}$ cardiac diseases & NA & NS & 29 \\
\hline Movement disorders * cardiac diseases & NA & NS & 84 \\
\hline
\end{tabular}

\section{DISCUSSION}

This study was conducted to evaluate the differential longitudinal effects of 96 chronic diseases, grouped into clusters, on cognition in a healthy aging population. The present results indicate that specific clusters of chronic diseases indeed were associated with a decline in one or both tested domains of cognitive functioning during a 12 year follow-up period.

The rate of decline on verbal memory and processing speed during the 12 years followup period was higher among diabetes patients, which is in line with previous studies, ${ }^{9}$. Although the RNH database does not make a clear distinction between type I and type II diabetes mellitus, only 11 out of 101 (10.9\%) diabetes patients (at 12 year follow-up) were diagnosed with diabetes before the age of forty. Hence, it can be stated that the conclusion drawn about the association between diabetes mellitus and cognition is primarily based on people diagnosed with diabetes mellitus type II. Other endocrine diseases appeared to be negatively related to processing speed, but not to verbal memory.

Ischemic heart disease and arrhythmias/heart failure were both negatively related to the two domains of cognitive functioning. Previous studies on the relationship between cardiovascular diseases and cognition have shown mixed results; some studies found no association ${ }^{9}$, while others found a negative association ${ }^{10,29}$. Other cardiovascular diseases, including hypertension and other peripheral vascular diseases, were only negatively associated with processing speed, which is in accordance with previous cross-sectional research?. Our findings also support the evidence for a negative association between cerebrovasculair disease and cognitive performance ${ }^{11}$. We found evidence of a decline on both cognitive domains among persons with malignancies. Previous research regarding this association is inconclusive. While some studies reported a negative association ${ }^{30}$, Comijs et al (2009) even reported a positive association between cancer and memory (delayed recall $)^{12}$.

Interestingly, the rate of memory and speed decline was higher among persons with gastro-intestinal diseases. A negative association between asthma/COPD/bronchitis and both cognitive domains was also found, which is in accordance with previous study on the association between $\mathrm{COPD}^{31} /$ chronic bronchitis $^{9}$ and cognitive decline. Persons with 
peptic ulcers were more prone to show a decline in processing speed as compared to healthy controls. Diseases of the urinary tract were also significantly related to processing speed. In contrast, being diagnosed with eczema/psoriasis/chronic skin ulcer only decreased one's performance on verbal memory.

Mood disorders as well as other mental disorders (including anxiety and schizophrenia) were only related to speed of processing. Contradictory, previous studies illustrated mood disorders to be related to numerous cognitive domains, including memory, speed, executive functioning and verbal fluency ${ }^{32}$. The lack of an association between mood disorders as well as other mental disorders and memory might be due to the fact that persons in the present study were a relatively healthy group, with no overt clinical signs of mental disorders (at baseline). It could be agued that since all statistical analyses were controlled for depressive complaints, this could have given an underestimation of the true effects of mood disorder. However, the same results were obtained when depressive complaints were not incorporated as a confounding variable.

Although Parkinson patients were expected to show a decreased performance in both cognitive domains, only a decrease in verbal memory performance was found. Since Parkinsonism was excluded at baseline and only 25 persons developed Parkinson in the 12years of follow-up, the current study might have lacked the power to detect a significant effect. Furthermore, persons who developed Parkinson in the course of the study were a relative healthy group as they needed to be in order to keep participating in the study notwithstanding their disease. Persons diagnosed with other movement disorders also showed a decline in processing speed. Since the LDST is a task that requires proper upper limb coordination, a worse performance of persons with movement disorders as compared to healthy control was expected.

Diseases of the eye were related to verbal memory as well as to processing speed since both tasks required proper vision. Diseases of the ear were also related to a decrease in processing speed. However, in a cross-sectional study by van Boxtel and colleagues diseases of the ear were only related to a decrease in verbal memory (1998) ${ }^{9}$. Migraine/headache, other diseases of the nervous system and other chronic respiratory diseases were not associated with either of the two cognitive functions.

To summarize, several disease clusters appear to be related to cognitive decline. Due to the explorative nature of this study, it is difficult at this stage to explain these associations. However, we believe that our research may help in finding unexpected common pathogenetic mechanisms, such as autoimmune responses, genetics, inflammation, immunological mechanisms or depressogenic actions. This unbiased approach may raise new hypotheses which may result in a better understanding of general concepts of comorbidity and frailty, in contrast to a disease-specific approach. Hence, the present results indicate that a standard classification of diseases based on overt sign and symptoms might be inconclusive.

Multimorbidity, or the co-occurrence of two ore more chronic diseases within one person, is common in general practice as evidenced through a prevalence of $55.2 \%$ in our study population. Previous research reported lower prevalence estimates ${ }^{3,33}$, which might be due to the limited number of medical conditions included in those studies ${ }^{2}$. Investigating 
the influence of disease combinations, on cognition showed that only the combination malignancies and movement disorders was significantly related to cognitive decline. Hence, persons suffering from a movement disorder as well as a malignancy perceive more cognitive decline than persons who are suffering from only one of these two disease clusters. Multimorbidity appeared to be less associated to cognitive decline than expected based on the negative influence of multimorbidity on other health-related outcomes. Hence, the present results indicate that specific disease clusters have a much more profound effect on cognition than multimorbidity.

All presented results were controlled for age, sex, depressive complaints and education which all appeared to be significantly related to cognitive functioning. In contrast to previous research, which suggested that the total number of morbid conditions was significantly related to memory, but not to speed of processing ${ }^{34}$, no association between total morbidity score and cognition was found in the present study. This dissimilarity might be the result of the inclusion of as much as 96 medical conditions, as compared with previous conducted studies, which in general included only a small selection of medical conditions. Since so many medical conditions were included, not all conditions will have the same profound effect on cognition. For example, a person diagnosed with only one chronic condition (e.g. dementia) can encounter much more cognitive decrements than a person diagnosed with multiple chronic conditions. Moreover, total cluster score was also considered as a possible confounder. The number of morbid clusters appeared not to be significantly related to memory or processing speed.

Since two neuropsychological tests were used as two distinct outcome measures (i.e. Visual Verbal Learning Test and Letter Digit Substitution Task), it might be argued that compound scores (several raw test scores being grouped into one score) would improve the robustness of the underlying cognitive construct. However, for reasons of parsimony the authors decided to only use the two abovementioned tests as a selection of all possible cognitive tests. Both tests are relatively robust, rather sensitive to age effects and are among the most widely used tests in clinical practice as well as in cognitive research ${ }^{24}$, which makes comparison the previously conducted studies rather straightforward.

The current study has several advantages over previous studies. First of all, this is the first study that incorporated as many as 96 chronic medical conditions grouped into different clusters. By doing so, a more complete inventory of the relation between medical conditions and cognition is studied. Second, this study provided longitudinal data with a follow-up of 12 years in a large population-based sample. Since cognitive decrements due to morbidity status are expected to develop in the course of many years, a realistic picture of the influence of morbidity status on cognitive decline emerges from the present study. Thirdly, we have evaluated the causal relationship between morbidity and cognition in a general practice based setting. Consequently, the results are more representative for the general population than results of studies conducted in smaller and less healthy homogeneous samples ${ }^{35}$. Moreover, in contrast to previous studies, the current study assessed medical status by using a general practice database instead of using self-reported medical status. Fourthly, although several studies already considered the relationship between morbidity and cognition, the majority, if not all, of these studies lacked data on 
disease duration. This drawback was overcome in the present study, since date of diagnosis was used as an indicator for the duration of exposure to the disease.

Despite the previous mentioned strengths, our findings must be interpreted in light of some possible limitations. Firstly, since the present study evaluated the effects of 96 chronic medical conditions on cognition, clustering was needed in order to conduct the statistical analyses. The clustering was based on the medical knowledge and consensus of three of the co-authors. It should be mentioned that this still remains a rather subjective method for clustering, which could have compromised the validity of the clusters. Furthermore, by evaluating the effects of clusters of medical conditions (instead of specific single medical conditions) on cognition, a potential strong association between a single disease and cognitive function may become diluted when mixed with the effects of other diseases within a cluster. Secondly, defining multimorbidity by a simple disease count might be overly simplistic for studies like the one conducted here. Evidence suggests that disease severity might yield a more accurate relationship between morbidity and cognitive functioning ${ }^{36}$. Thirdly, the total number of conditions registered in the RNH database reflects the GPs perspective of the health status and relevant health problems of his patients. As a result, some health problems may still be missing because the patient did not report them to the GP or because the GP did not judge them to be clinically significant. The number of undocumented health problems, however, appears to be rather small ${ }^{20}$. Furthermore, the registered date of diagnosis may not always correspond fully to the actual onset of a disease ${ }^{19}$, which could have resulted in a slight underestimation of disease duration.

The current results underscore earlier claims that morbidity status can be considered as a possible mediating factor in cognitive dysfunction ${ }^{9}$. Since the present study needs to be viewed as a first explorative study on the association between chronic medical diseases and cognitive decline, it is important to replicate the present research. Considering the large number of patients who suffer from multimorbidity, a more detailed exploration of the impact of chronic diseases on cognition becomes even more important the next few decades. Evaluating the influence of multimorbidity on "better" or overall desirable cognitive functioning, and "worse" or undesirable cognitive functioning might be useful for translating the present results into prevention and intervention programs to reduce cognitive decline mediated by morbidity status. 


\section{REFERENCES}

1. M. van den Akker, F. Buntinx, and J. Knottnerus. Comorbidity or multimorbidity: What's in a name. A review of literature. Eur. J. Gen. Pract., 1996;2:65-70.

2. M. van den Akker, F. Buntinx, J.F. Metsemakers, S. Roos, and J.A. Knottnerus. Multimorbidity in general practice: prevalence, incidence, and determinants of co-occurring chronic and recurrent diseases. J. Clin. Epidemiol., 1998;51(5):367-375.

3. A. Marengoni, B. Winblad, A. Karp, and L. Fratiglioni. Prevalence of chronic diseases and multimorbidity among the elderly population in Sweden. Am. J. Public Health, 2008;98(7):1198-1200.

4. M. Fortin, G. Bravo, C. Hudon, A. Vanasse, and L. Lapointe. Prevalence of multimorbidity among adults seen in family practice. Annuals of Family Medicine, 2005;3(3):223-228.

5. A.V. Perruccio, J.D. Power, and E.M. Badley. The relative impact of 13 chronic conditions across three different outcomes. J. Epidemiol. Community Health, 2007;61(12):1056-1061.

6. M. Fortin, G. Bravo, C. Hudon, L. Lapointe, J. Almirall, M.F. Dubois, and A. Vanasse. Relationship between multimorbidity and health-related quality of life of patients in primary care. Qual. Life. Res., 2006;15(1):83-91.

7. I. Bergman, M. Blomberg, and O. Almkvist. The importance of impaired physical health and age in normal cognitive aging. Scand. J. Psychol,. 2007;48(2):115-125.

8. S.E. Vermeer, N.D. Prins, T. den Heijer, A. Hofman, P.J. Koudstaal, and M.M. Breteler. Silent brain infarcts and the risk of dementia and cognitive decline. N. Engl. J. Med., 2003;348(13):1215-1222.

9. M.P. van Boxtel, F. Buntinx, P.J. Houx, J.F. Metsemakers, A. Knottnerus, and J. Jolles. The relation between morbidity and cognitive performance in a normal aging population. J. Gerontol. A Biol. Sci. Med. Sci., 1998;53(2):M147-154.

10. P.C. Elwood, J. Pickering, A. Bayer, and J.E. Gallacher. Vascular disease and cognitive function in older men in the Caerphilly cohort. Age Ageing, 2002;31(1):43-48.

11. M.G. Dik, D.J. Deeg, L.M. Bouter, E.H. Corder, A. Kok, and C. Jonker. Stroke and apolipoprotein E epsilon 4 are independent risk factors for cognitive decline: A population-based study. Stroke, 2000;31(10):2431-2436.

12. H.C. Comijs, D.M. Kriegsman, M.G. Dik, D.J. Deeg, C. Jonker, and W.A. Stalman. Somatic chronic diseases and 6-year change in cognitive functioning among older persons. Arch. Gerontol. Geriatr., 2009;48(2):191-196.

13. D.W. Desmond, J.T. Moroney, M.C. Paik, M. Sano, J.P. Mohr, S. Aboumatar, C.L. Tseng, S. Chan, J.B. Williams, R.H. Remien, W.A. Hauser, and Y. Stern. Frequency and clinical determinants of dementia after ischemic stroke. Neurology, 2000;54(5):1124-1131.

14. A. Ott, R.P. Stolk, F. van Harskamp, H.A. Pols, A. Hofman, and M.M. Breteler. Diabetes mellitus and the risk of dementia: The Rotterdam Study. Neurology, 1999;53(9):1937-1942.

15. L. Patrick, P. Gaskovski, and D. Rexroth. Cumulative illness and neuropsychological decline in hospitalized geriatric patients. Clin. Neuropsychol., 2002;16(2):145-156.

16. S. Goebeler, M. Jylha, and A. Hervonen. Self-reported medical history and self-rated health at age 90. Agreement with medical records. Aging Clin. Exp. Res., 2007;19(3):213-219.

17. D.M. Kriegsman, B.W. Penninx, J.T. van Eijk, A.J. Boeke, and D.J. Deeg. Self-reports and general practitioner information on the presence of chronic diseases in community dwelling elderly. A study on the accuracy of patients' self-reports and on determinants of inaccuracy. J. Clin. Epidemiol., 1996;49(12):1407-1417.

18. J.J. Jolles, P.J. Houx, M.P.J. van Boxtel, and R.W.H.M. Ponds. The Maastricht Aging Study: Determinants of cognitive aging. 1995, Neuropsych Publishers Maastricht: Maastricht.

19. J.F.M. Metsemakers, P. Höppener, J. Knottnerus, and C. Limonard. Computerized health information in the Netherlands: a registration network of family practices. Br. J. Gen. Pract., 1992;42(356):102106. 
20. J.F. Metsemakers, J.A. Knottnerus, G.J. van Schendel, R.J. Kocken, and C.B. Limonard. Unlocking patients' records in general practice for research, medical education and quality assurance: the Registration Network Family Practices. Int. J. Biomed. Comput., 1996;42(1-2):43-50.

21. C.c.o. WONCA. ICPC International Classification of Primary Care. 1987, Oxford: Oxford Univesity Press.

22. J.F.M. Metsemakers, J.A. Knottnerus, G.J. van Schendel, R.J. Kocken, and C.B. Limonard. Unlocking patients' records in general practice for research, medical education and quality assurance: the Registration Network Family Practices. Int. J. Biomed. Comput., 1996;42(1-2):43-50.

23. W. Van der Elst, M.P. van Boxtel, G.J. van Breukelen, and J. Jolles. Rey's verbal learning test: normative data for 1855 healthy participants aged 24-81 years and the influence of age, sex, education, and mode of presentation. J. Int .Neuropsychol. Soc., 2005;11(3):290-302.

24. M.D. Lezak, D.B. Howieson, and D.W. Loring. Neuropsychological assessment. 2004, New York: OXFORD university press.

25. W. van der Elst, M.P. van Boxtel, G.J. van Breukelen, and J. Jolles. The Letter Digit Substitution Test: normative data for 1,858 healthy participants aged 24-81 from the Maastricht Aging Study (MAAS): influence of age, education, and sex. J. Clin. Exp. Neuropsychol, 2006;28(6):998-1009.

26. S.E. de Bie. Standaardvragen 1987: Voorstellen van uniformering van vraagstellingen naar achtergrondkenmerken en interviews. 2nd ed. 1987, Leiden, The Netherlands: Leiden University Press.

27. L.R. Derogatis and P.A. Cleary. Factorial invariance across gender for the primary symptom dimensions of the SCL-90. Br. J. Soc. Clin. Psychol., 1977;16(4):347-356.

28. F.E.S. Tan. Best practices in analysis of longitudinal data: a multilevel approach, in Best practices in quantitative methods, Osborne J.W., Editor. 2008: London.451-471.

29. S.R. Waldstein, C.F. Tankard, K.J. Maier, J.R. Pelletier, J. Snow, A.W. Gardner, R. Macko, and L.I. Katzel. Peripheral arterial disease and cognitive function. Psychosom. Med., 2003;65(5):757-763.

30. E.A. Kvale, O.J. Clay, L.A. Ross-Meadows, J.S. McGee, J.D. Edwards, F.W. Unverzagt, C.S. Ritchie, and K.K. Ball. Cognitive speed of processing and functional declines in older cancer survivors: an analysis of data from the ACTIVE trial. Eur. J. Cancer Care, 2009.

31. S. Borson, J. Scanlan, S. Friedman, E. Zuhr, J. Fields, E. Aylward, R. Mahurin, T. Richards, Y. Anzai, M. Yukawa, and S. Yeh. Modeling the impact of COPD on the brain. Int. J. Chron. Obstruct. Pulmon. Dis., 2008;3(3):429-434.

32. M. Talarowska, A. Florkowski, P. Galecki, A. Wysokinski, and K. Zboralski. [Cognitive functions and depression]. Psychiatria polska, 2009;43(1):31-40.

33. H.C. Britt, C.M. Harrison, G.C. Miller, and S.A. Knox. Prevalence and patterns of multimorbidity in Australia. Med. J. Aust., 2008;189(2):72-77.

34. H.T. Nguyen, M.K. Evans, and A.B. Zonderman. Influence of medical conditions on executive and memory functions in low socioeconomic status African Americans. Arch. Clin. Neuropsychol., 2007;22(6):689-698.

35. A. Marengoni, D. Rizzuto, H.X. Wang, B. Winblad, and L. Fratiglioni. Patterns of chronic multimorbidity in the elderly population. J. Am. Geriatr. Soc. 2009;57(2):225-230.

36. R. Rozzini, G.B. Frisoni, L. Ferrucci, P. Barbisoni, T. Sabatini, P. Ranieri, J.M. Guralnik, and M. Trabucchi. Geriatric Index of Comorbidity: validation and comparison with other measures of comorbidity. Age Ageing, 2002;31(4):277-285. 


\section{APPENDIX I}

ICPC codes and the corresponding clusters

\begin{tabular}{|c|c|}
\hline Cluster description & ICPC code \\
\hline All malignancies & $\begin{array}{l}\text { A79, B72, B73, D74, D75, D76, D77, F74, N74, } \\
\text { R84, R85, S77, T71, U75, U76, U77, W72, X75, } \\
\text { X76, X77, Y77, Y78 }\end{array}$ \\
\hline Peptic ulcers & $\mathrm{D} 85, \mathrm{D} 86$ \\
\hline Other chronic gastro-intestinal diseases & D92, D93, D94, D97 \\
\hline Diseases of the eye & F83, F84, F94 \\
\hline Diseases of the ear & H83, H86, \\
\hline Ischemic diseases & $\mathrm{K} 74, \mathrm{~K} 75, \mathrm{~K} 76$ \\
\hline Pulmonary embolism and phlebitis & K93, K94 \\
\hline Cerebrovascular diseases & K89, K90, K91 \\
\hline Arrhythmias \& heart failure & K78, K79, K80, K77, K82, K83 \\
\hline Other cardiovascular diseases & K87, K92 \\
\hline Movement disorders & L84, L85, L88, L89, L90, L91, L95, L98 \\
\hline Parkinsonism & N87 \\
\hline Migraine \& Headache & N89, N90 \\
\hline Other diseases of the nervous system & N92, N70, N86, N88 \\
\hline Mood disorders & P76, P73 \\
\hline Alzheimer & P70 \\
\hline Other mental disorders & P28, P71, P72, P74, P75, P77, P79, P80, P98 \\
\hline Asthma, COPD and bronchitis & R91, R95, R96, \\
\hline Other chronic respiratory diseases & R70, R75, R97 \\
\hline Eczema, psoriasis and chronic skin ulcer & S87, S91, S97 \\
\hline Endocrine diseases & Т85, Т86, Т92, Т93, Т99 \\
\hline Diabetes mellitus (Type I and II) & T90 \\
\hline Diseases of the urinary tract & U04, U85, U88 \\
\hline
\end{tabular}





\section{CHAPTER 4}

The effect of multimorbidity on health related functioning: temporary or persistent? Results from a longitudinal cohort study

Under review as:

S. Aarts, M. van den Akker, H. Bosma, F.E.S. Tan, F.R.J. Verhey, J.F.M. Metsemakers and M.P.J. van Boxtel 


\section{ABSTRACT}

\section{Background}

Multimorbidity is known for its negative effects on health related functioning. It remains unclear if these effects are stable over time. The aim was to investigate if the relation between single morbidity/multimorbidity and health related functioning, as measured by the SF-36, is temporary or persistent.

\section{Methods}

Data were collected as part of the Maastricht Aging Study (MAAS), a prospective study into the determinants of cognitive aging. Participants $(n=1,184), 24-81$ years old, were recruited from a patient database in primary care (Registration Network Family Practices). Morbidity status (i.e. healthy, single morbidity or multimorbidity) and the Short Form Health Survey (SF-36) were both assessed at baseline, at 3-and 6-year follow-up.

Results

At baseline but not at 3-and 6-year follow-up, participants with single morbidity reported poorer physical functioning than their healthy counterparts. Multimorbidity was associated with poorer physical functioning at all measurements. Participants with multimorbidity showed a steep decrease in physical functioning between 3-and 6-year follow-up. Multimorbidity appeared to be unrelated to mental functioning. At baseline and at 3-year follow-up, participants who had a change in morbidity status reported more decline in physical functioning than their healthy counterparts.

\section{Conclusions}

The decline in physical functioning that accompanies multimorbidity is persistent and may even increase over time. People, who acquire one or more diseases during 3-year follow-up, already showed a decreased physical functioning at baseline than people who remained healthy during these years. Post-hoc analyses, using the SCL-90 as an outcome measure, did show that multimorbidity was related to depressive and anxiety complaints. However, these complaints seem to decline over time 


\section{INTRODUCTION}

Multimorbidity, the co-occurrence of two or more chronic diseases within one person ${ }^{1}$, is the rule rather than the exception ${ }^{2,3}$. In a previously conducted study based on a general practice cohort, using a broad nosological spectrum of disease entities, 52 percent of the participants (aged between 21 to 84 years) were suffering from multimorbidity ${ }^{4}$. As a result of aging populations and advances in medical care, the prevalence of multimorbidity is expected to increase even more the upcoming decades. Since a higher prevalence of multimorbidity will lead to higher health care utilization and thus to higher health care expenditure ${ }^{5}$, the influence of multimorbidity on society will be marked.

The questions remains whether the consequences of single morbidity and multimorbidity on indicators of physical ${ }^{6}$ and mental health ${ }^{7}$ are temporary or persistent over time. For example, it might be argued that a long-term experience with a chronic illness shows a different relationship with adverse health outcomes than a recently acquired chronic medical condition. The period directly after a newly acquired disease may lead to significant decrease in self-reported physical and mental health related factors ${ }^{8}$ (e.g. decreased quality of life and increased levels of disability ${ }^{9}$ ) while these consequences might diminish or even disappear when a person has adjusted to the newly acquired illness. Health problems caused by single morbidity or multimorbidity could also be permanent or could even increase over time. Untangling the longitudinal association between single morbidity and multimorbidity on one side and health related functioning on the other is a crucial first step towards the development of adequate disease management that could ameliorate functional problems caused by multimorbidity.

The present study provides the first data documenting possible changes in health related functioning caused by single morbidity and multimorbidity. The following two research questions were addressed: 1. Are the effects of single morbidity and multimorbidity on health related functioning status stable over time? 2. Does a change in morbidity status lead to a drop in health related functioning?

\section{METHODS}

The present study was based on two cohorts: The Registration Network Family Practices $(\mathrm{RNH})$ and the Maastricht Aging Study (MAAS). Participants in MAAS were originally recruited from the Registration Network Family Practices, which contains demographic and medical information for over 120.000 patients inhabiting in the South of Limburg, the Netherlands ${ }^{10}$.

\section{Registration Network Family Practices (RNH)}

Overall morbidity status of all MAAS participants at baseline, 3- and 6 year follow-up was retrieved from the RNH database. The RNH is a continuously updated database, which contains the electronic medical records of patients from 21 family practices, in which 65 general practitioners (GPs) are participating . This database includes all relevant current and past health problems. A health problem is defined as 'anything that has required, does 
or may require health care management and has affected or could significantly affect a person's physical or emotional well-being'. A diagnosis is made by the GP or, in case of complex conditions, by a medical specialist to whom a patient has been referred. The RNH database contains health problems that are only coded by the GP when they are permanent (no recovery expected), chronic (duration longer than 6 months) or recurrent (more than three recurrences within 6 months), or when they have lasting consequences for the functional status or prognosis of the patient. Problems are coded in a standardized fashion, according to the International Classification of Primary Care (ICPC) ${ }^{11}$, using the criteria of the International Classification of health in Primary Care (ICHPPC-2) and other more current guidelines of the Dutch College of GPs.

All patients included in the RNH database have been informed about the potential anonymous use of their health information. Inclusion in the RNH registry ends following migration or death; however, if a patient does not agree to the use of his/her health information, the inclusion of this patient in the RNH database can be stopped. Sociodemographical characteristics of samples in other studies using the RNH database was shown to be comparable to the Dutch population ${ }^{12}$.

\section{Maastricht Aging Study (MAAS)}

In 1992, 10396 people were recruited from the RNH database of whom 4490 were willing to participate (43.2\%) in MAAS, 3531 refused participation (34\%), and 2375 did not respond $(22.8 \%)$. Since MAAS was aimed to investigate healthy cognitive aging in a wide age range, people aged 24 to 81 years were included. Participants were excluded from baseline participation when medical records from the $\mathrm{RNH}$ database contained the following conditions which are known to interfere with cognition: history of coma, cerebrovascular disorder, tumour of the nervous system, multiple sclerosis, Parkinsonism, epilepsy, dementia, organic psychosis, schizophrenia, affective psychosis, mental retardation or congenital malformation of the nervous system ${ }^{13}$. The 4490 eligible participants were screened by telephone for medical conditions not documented in the $\mathrm{RNH}$ database (i.e. history of transient ischemic attack, brain surgery, electroconvulsive therapy, daily psychotropic drug use and haemodialysis for renal failure). Of the remaining 4189 participants (301 were excluded based on the telephone screening), 1823 were randomly selected for participation in the test program of MAAS. Of them, 1353 individuals also participated in the 6 year follow-up test programme.

The MAAS study consists of five measurements: baseline, 3-year follow-up, 6-year follow-up, 9-year follow-up and 12-year follow-up. Since the outcome variable of the present study was not measured at baseline and the 3-year follow-up only included participants aged 55 years and older, the 6-year follow-up was set as baseline assessment for the present study. The 9-and 12 year follow-up measurements were set as respectively, the first (i.e. 3-years follow-up) and second follow-up (i.e. 6-years follow-up) measurement. Participants who had no information regarding the SF-36 or who lacked morbidity status in $\mathrm{RNH}(\mathrm{n}=169)$ (e.g. because of movement and mortality) were excluded from all the analyses. 


\section{Dependent Variables}

The Short Form Health Survey (SF-36), also known as the RAND-36, is a widely used generic measures aimed as assessing health related functioning, which relies upon patient self-reports ${ }^{14}$. The 36 generic and coherent questions are aimed at covering the full spectrum of health related functioning and are grouped into eight dimensions of health (i.e. physical function, role-physical, bodily pain, general health, energy/vitality, social function, role-emotional, and mental health). These dimensions are indicators for behavioural function and dysfunction, distress and well-being, and self-rated health. The present study made use of the mental and physical health subscales as defined by Ware and Sherbourne ${ }^{14}$. For both subscales a higher score is indicative for better health related functioning. The SF36 was administered at all three follow-up measurements.

\section{Independent Variables}

Overall morbidity status (i.e. healthy, single morbidity or multimorbidity) of all MAAS participants was assessed by looking at the medical records at baseline and at the two follow-up measurements. Letting morbidity status vary at every time point resulted in ten mutually exclusive groups (see Appendix I). However, the majority of these groups consisted only of a very small number of participants which resulted in a lack of power. Consequently, the present study focused on the following three groups: people who were healthy (i.e. no disease present) on all three measurements, people with single morbidity (i.e. one disease present) on all three measurements and people with multimorbidity (i.e. two or more diseases present) on all three measurements.

Based on medical literature ${ }^{15}$ and on clinical experience, the following chronic medical conditions were included: malignancies, movement disorders, chronic respiratory diseases, cardiovascular diseases (heart failure, ischemic diseases, arrhythmias and cerebrovascular diseases), endocrine diseases, neurological disturbances and psychological disorders (see Appendix I). The medical conditions on which exclusion for MAAS participation was based were also included to monitor incident cases after baseline.

Since the present study only included medical conditions that were judged by the GP to be permanent, chronic, recurrent or when they have lasting consequences for the functional status or prognosis of the patient, the assumption was made that people who were suffering from single morbidity or multimorbidity could not return to a healthy status and people suffering from multimorbidity could not be suffering from single morbidity on a follow-up measurement. In order to evaluate the effects of a change in morbidity status on one side and physical and mental health on the other, an additional statistical analysis was conducted in which participants who were healthy at baseline as well as at 3 year follow-up were compared with participants who were healthy at baseline but who had single morbidity or multimorbidity at the 3 year follow-up measurement.

\section{Possible Confounders}

Potential confounders were taken into account: age, sex, educational level (low, medium or high; low was defined as primary school and/or lower vocational education, medium as 
secondary school and/or medium level vocational education, high as higher vocational education or university degree) ${ }^{16}$, living arrangements (two categories; living alone vs. living together), current smoking behaviour (yes or no) and alcohol intake (excessive drinker: yes/no; excessive was defined as more than 21 glasses per week for men and more than 14 glasses per week for women).

\section{Statistical Analyses}

As the independent variable and the outcome measures were measured three times, data were analyzed with Linear Mixed Models. Since measurements within subjects are more alike than measurements between subjects, there is correlation between the repeated measurements. This within-subjects variation, that accounts for changes within each subject through repeated measurements in time, and the between-subjects variation, accounting for differences between the subjects performance, can be established by a multilevel approach ${ }^{17}$. Moreover, missing data may depend on the observed covariates and outcome measures (missing at random). When evaluating the plots of the residuals and when taking the Maximum Likelihood ratio into account, a marginal model with the unstructured covariance structure provided the best fit ${ }^{17}$.

The multilevel models included fixed terms for morbidity status, follow-up (time of measurement), age, sex, education, living arrangement, smoking behaviour and alcohol intake. Age, sex and education were included in all the analyses irrespective of significance. The remaining covariates were only included in the model and reported if they significantly influenced the outcome under study $(\mathrm{p} \leq 0.05)$. Interaction effects between morbidity status and follow-up were evaluated in order to detect possible changes in physical and mental health which might be due to single morbidity or multimorbidity. The additional statistical analysis (i.e. participants who were healthy at baseline as well as at 3 year followup were compared with participants who were healthy at baseline but who had single morbidity or multimorbidity at the 3 year follow-up measurement) were analyzed using Multiple Linear Regression Analyses. P-values of 0.05 or less were considered statistically significant. All analyses were conducted with the SPSS statistical software package version 17.0 for Windows (SPSS Inc., Chicago, IL, USA). Graphs (included in the Appendix) will be presented using z-scores with a mean of zero and a standard deviation of one.

\section{RESULTS}

Participants $(n=169)$ who were excluded from all the analyses were older $(p<0.001)$, less educated $(\mathrm{p}<0.001)$ and more likely to be female $(\mathrm{p}=0.047)$. Moreover, the excluded participants were less likely to be healthy or to be suffering from single morbidity, but were more likely to be suffering from multimorbidity $(\mathrm{p}<0.001)$. Descriptive characteristics of the study sample $(n=1184)$ are reported in Table 1 . Of all participants, $19.6 \%$ suffered from single morbidity at all three measurements while $35.5 \%$ suffered from multimorbidity at all three measurements; $83.6 \%(n=990)$ of all participants had the same morbidity status throughout the three measurements. Only participants who had no change in morbidity status were included for the analysis leading to a total of 990 participants (see Appendix II). 
At baseline but not at 3-and 6-year follow-up, participants with single morbidity reported significantly more decline in physical functioning than their healthy age mates (Table 2 and Figure 1.a.). Between baseline and 3-year follow-up, people with single morbidity showed less decline in physical functioning than healthy participants. No effect of morbidity status (i.e. healthy vs. single morbidity) was found between 3-and 6-year follow-up.

Table 1. Descriptive characteristics of the study population at baseline $(\mathrm{N}=1184)$

\begin{tabular}{lc}
\hline Characteristics & \\
\hline Mean age in year (SD) & $55.4(15.2)$ \\
Gender: females (\%) & $573(48.4)$ \\
Morbidity status & \\
Healthy & $338(28.5)$ \\
Single morbidity & $232(19.6)$ \\
Multimorbidity & $420(35.5)$ \\
Education & \\
Low (\%) & $371(31.3)$ \\
Medium (\%) & $441(37.2)$ \\
High (\%) & $320(27.0)$ \\
Living arrangement (\% living with family) & $903(76.3)$ \\
Smoking (\% yes) & $178(21.3)$ \\
Alcohol consumption (\% yes) & $107(9.0)$ \\
\hline
\end{tabular}

Morbidity status refers to participants who had the same morbidity status at all three measurements $16.4 \%(\mathrm{n}=194)$ of the participants did show a change in morbidity status during the 6 year follow-up.

On all three measurements, participants with multimorbidity reported significantly lower physical functioning than their healthy counterparts. Moreover, between 3-and 6-year follow-up, there was a significant interaction between morbidity status (i.e. healthy vs. multimorbidity) and follow-up, which suggest that participants with multimorbidity showed a significant steeper decrease in physical functioning than healthy participants. Between baseline and 3-year follow-up, no effect of morbidity status was found. Higher age and low education were significantly related to lower physical functioning.

At 6 year follow-up, a significant relation between single morbidity and mental functioning, as measured by the SF-36, was detected. (Table 2 and Figure 1.b.). No significant interaction between morbidity status (i.e. healthy vs. single morbidity) and follow-up measurement was found. On all three measurements, multimorbidity was not significantly related to mental functioning. Moreover, no significant interactions between morbidity status (i.e. healthy vs. multimorbidity) and follow-up measurement were found. Lower age, female sex and lower education were all significantly related to lower mental functioning.

Participants with a change in morbidity status (i.e. who were healthy at baseline and had either single morbidity or multimorbidity at 3-year follow-up; $n=47$ ) between baseline and 3-year follow-up reported significant lower physical functioning than their healthy counterparts $(n=294)$ (Table 3 and Figure 2.a.). These two groups did not differ regarding mental functioning (Table 3 and Figure 2.b). On the physical as well as on the mental subscale no interaction effect between morbidity status and follow-up measurement was 


\section{Chapter 4}

found; both groups showed the same change in physical and mental functioning during the 3 -year follow-up. High age and lower education were significantly related to lower physical functioning. Lower education was also significantly related to lower mental functioning. Although all the abovementioned associations were adjusted for age, sex and educational level, all associations were significantly related to the outcome at hand when no adjustment had taken place.

Table 2. Multilevel analyses for the association between single morbidity and multimorbidity on one side and mental and physical health on the other, adjusted for age, gender and education

\begin{tabular}{|c|c|c|c|c|}
\hline & \multicolumn{2}{|c|}{ Physical health } & \multicolumn{2}{|c|}{ Mental health } \\
\hline & $\begin{array}{l}\text { Parameter } \\
\text { estimate }\end{array}$ & P-value & $\begin{array}{l}\text { Parameter } \\
\text { estimate }\end{array}$ & P-value \\
\hline Age & -0.148 & $<0.001$ & 0.037 & 0.041 \\
\hline Gender & -0.853 & 0.081 & -1.474 & 0.002 \\
\hline Education & 1.616 & $<0.001$ & 1.166 & $<0.001$ \\
\hline \multicolumn{5}{|l|}{ Single morbidity } \\
\hline Baseline & -2.030 & 0.013 & -0.008 & 0.993 \\
\hline 3 year follow-up & -0.273 & 0.722 & 0.971 & 0.247 \\
\hline 6 year follow-up & -0.907 & 0.262 & -0.552 & 0.048 \\
\hline \multicolumn{5}{|l|}{ Multimorbidity } \\
\hline Baseline & -5.643 & $<0.001$ & -0.839 & 0.300 \\
\hline 3 year follow-up & -5.505 & $<0.001$ & -1.090 & 0.163 \\
\hline 6 year follow-up & -6.74 & $<0.001$ & -1.038 & 0.169 \\
\hline \multicolumn{5}{|l|}{ Interaction } \\
\hline $\begin{array}{l}\text { Single morbidity* time } \\
\text { (baseline and } 3 \text { year follow-up) }\end{array}$ & 1.756 & 0.027 & 0.979 & 0.302 \\
\hline $\begin{array}{l}\text { Single morbidity } * \text { time } \\
\text { ( } 3 \text { year FU and } 6 \text { year follow-up) }\end{array}$ & 0.634 & 0.364 & 1.524 & 0.092 \\
\hline $\begin{array}{l}\text { Multimorbidity* time } \\
\text { (baseline and } 3 \text { year follow-up) }\end{array}$ & -0.138 & 0.843 & -0.253 & 0.0761 \\
\hline $\begin{array}{l}\text { Multimorbidity * time } \\
\text { ( } 3 \text { year FU and } 6 \text { year follow-up) }\end{array}$ & 1.231 & 0.049 & -0.054 & 0.946 \\
\hline
\end{tabular}

Note: the reference group consisted of healthy participants 
Table 3. Multilevel analyses for the association between a change in morbidity status (between baseline and 3 year follow-up) and mental and physical health, adjusted for age, gender and education

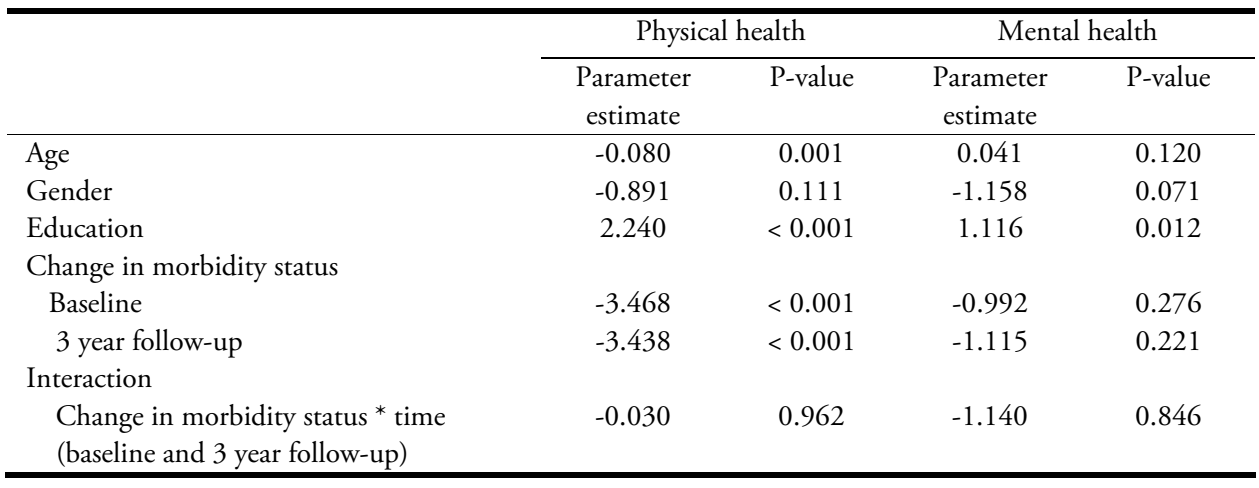

The reference group consisted of participants who were healthy at baseline and at 3 year follow-up; i.e. no change in disease status (participants who were healthy at baseline and at 3 year follow-up) vs. a change in disease status (participants who were healthy at baseline and had single morbidity or multimorbidity at 3 year follow-up.).

\section{DISCUSSION}

The present study describes the possible changes in health related functioning, divided in physical and mental functioning, as a function of morbidity status. Apart from an negative association between single morbidity and physical functioning on baseline, which might be due to a statistical phenomenon called 'regression to the mean' (i.e. the healthy participants who report an 'extreme' value on the SF-36 on the first measurement will tend to be closer to the mean at the 3 year follow-up), single morbidity appears to be unrelated to physical functioning. This is in contrast with a previous study conducted by Galenkamp and colleagues that claimed that the first chronic medical condition increases the chance of poor health functioning more than each co-occurring disease ${ }^{18}$. However, while cross-sectional study assessed self-reported general health (i.e. based on the question: "How is your health in general?"), our longitudinal study made a clear distinction between physical and mental functioning as assessed by the SF-36. Hence, comparison between this study and the present one is not straightforward.

At all three measurements, participants with multimorbidity did report poorer physical functioning than their healthy counterparts, which is in congruence with previous research $^{18,19}$. Moreover, people with multimorbidity tended to show a steeper decrease in physical functioning between 3-and 6-year follow-up as compared to their healthy counterparts. Hence, in contrast to people with single morbidity, people with multimorbidity may not adjust to the challenging physical demands that complement their illnesses which may result in an ongoing decrease in physical functioning. People who are suffering from multimorbidity are also more prone to acquire a new medical condition ${ }^{20}$, which could have further decreased their physical functioning.

In order to evaluate the effects of a change in morbidity status on one side and physical and mental functioning on the other, another analysis was conducted in which participants 
who were healthy at baseline and at 3-year follow-up were compared with participants who were healthy at baseline but who had single morbidity or multimorbidity at 3-year followup. At baseline, participants who showed a change in morbidity status (i.e. who were healthy at baseline and had either single morbidity or multimorbidity at 3-year follow-up) reported significant lower physical functioning than their healthy counterparts. This effect was still present at 3-year follow-up. These results indicate that at baseline, when both groups were still healthy, those individuals who acquired one or more medical conditions in the subsequent 3 year already experience lower physical functioning than those who remained healthy during the 3-year follow-up. Hence, it might be argued that a decrease in physical functioning is already present far before a disease diagnose is made by a GP or a specialist. The lack of an interaction effect between the two groups and follow-up indicates that the acquisition of one or more medical conditions did not lead to a sudden decrease in physical functioning.

Our results are in congruence with a study by Rijken and colleagues (2005) which concluded that comorbidity in chronic diseased patients was not significantly related to mental functioning as measured by the SF- $36^{18}$. Moreover, a study by Fortin and coworkers (2006) also indicated that multimorbidity causes greater deterioration in physical functioning than in mental functioning, as measured by the SF-36. Hence, the question might rise whether SF-36 is a sensitive index to detect psychological complaints caused by single morbidity or multimorbidity in a normal population. Hence, post-hoc analyses in which the effects of single morbidity and multimorbidity on the depression and anxiety subscales of the Symptom CheckList-90 (SCL-90; a higher score is indicative for more depressive and anxiety related complaints) were conducted (see appendix IV for table and figures). At baseline, but not at 3-and 6-year follow-up, people with multimorbidity reported significantly more depressive complaints than their healthy counterparts. At baseline, people with multimorbidity also reported significantly more anxiety complaints than their healthy counterparts. While this effect, although smaller, was still significant at 3-year follow-up, it was absent at 6-year follow-up. No differences between healthy people and people with single morbidity were found. Female sex was significantly related to more depressive complaints; lower education was significantly related to more complaints of depression and anxiety. The results of these post-hoc analyses indicate that, although multimorbidity is related to elevated levels of depressive and anxiety complaints, these tend to decline over time. It might be argued that people with multimorbidity eventually learn to adapt to their acquired illnesses which in turn might lead to a decrease in feelings of depression and anxiety. Moreover, the use of the SCL-90 yielded different results than using the SF-36. This might be due to the fact that, while the SF-36 is an index of subjective general health and probes somatic and psychosomatic health functioning, the SCL-90 subscales are more dedicated checklists aimed at evaluating psychological and psychopathological complaints. Such indexes may therefore be more sensitive for psychological distress that is caused by multimorbidity ${ }^{21}$.

The current study has several advantages over previously conducted studies. Firstly, although several studies already considered the relationship between (multi)morbidity on one side and mental and physical functioning on the other, the present study is the first to 
evaluate the stability of these negative consequences. Secondly, by including 96 medical conditions, a more complete inventory of the effect of morbidity and multimorbidity on mental and physical functioning could be made, in contrast to previous conducted studies $^{18}$. Thirdly, this study provides longitudinal data based on a large population-based sample. Since changes in health related functioning caused by medical conditions are expected to develop in the course of weeks, months and year, a realistic picture of the influence of (multi)morbidity status on mental and physical functioning has been provided. Fourthly, the causal relationship between multimorbidity and general health was investigated in a general practice based sample with a large age range. Consequently, the results are more representative for the general population than results of studies conducted in smaller, less homogeneous or clinical samples. Moreover, the current study made use of medical conditions diagnosed by general practitioners instead of using self-reported medical status.

The current results should also be viewed in light of some possible limitations. Firstly, defining multimorbidity by a simple disease count might be overly simplistic for studies such as the present one. Evidence suggests that disease severity, which could not be evaluated in the present study, might yield a more accurate relationship between multimorbidity and relevant health outcomes ${ }^{22}$. Secondly, it cannot be ruled out that patients could be cured from a few of the included medical conditions. However, since the present study only included medical conditions that were perceived by the GP as permanent, chronic, recurrent or having lasting consequences, this percentage is expected to be rather small. Thirdly, since the 6 year follow-up of the MAAS was set as an artificial baseline, it could be argued that the 'healthy ill' are overrepresented in the current study. This 'survivor effect' may have lead to underestimations of the obtained associations. Consequently, the finding that the association between single morbidity and multimorbidity on one side and mental health on the other diminishes over time, may not be generalized to more 'disadvantaged' individuals with single morbidity or multimorbidity.; the negative association between morbidity status and physical health may be more profound in these individuals.

Despite these limitations the present study provides the first data documenting if the effects of single morbidity and multimorbidity on health related functioning are temporary or persistent. The present results indicate that the decrease in self-perceived physical functioning, caused by multimorbidity, might be permanent. Moreover, the association between single morbidity and multimorbidity on one side and mental health, as measured by the SCL-90, on the other seems to decline over time. In conclusion, our results suggest that the association between multimorbidity and physical functioning may be permanent while the association between multimorbidity and mental health seems to decline over time. Since poor physical functioning is known to underlie several adverse outcomes, including hospitalization and nursing home admission ${ }^{23}$, appropriate disease management that is aimed at amelioration or even prevention of physical problems is needed.

Because several morbidity groups included only a small number of participants (see Appendix I), evaluating the association between these groups and the outcome at hand appeared unfeasible. Hence, in order to evaluate the association between all these morbidity 
groups on one side and physical and mental functioning on the other, the present study should be repeated using a larger sample size. Moreover, repeating the present study in older individuals (e.g. aged 70 and over) may also yield different results.

The question remains how the decrease in physical functioning influences the daily living of people with multimorbidity. Do the present results indicate that people with multimorbidity perceive hindrance in their activities of daily life (e.g. bathing, walking etc) or can they still carry out these activities as well as their healthy counterparts? Since the SF36 is not a diagnostic instrument, it is difficult to obtain a validated cut-off score on which clinical relevant conclusions can be based. Hence, the stability of physical and mental health, caused by disability status, should be investigated since disability might be a better predictor for health related functioning. 


\section{REFERENCES}

1. M. van den Akker, F. Buntinx, and J. Knottnerus. Comorbidity or multimorbidity: What's in a name. A review of literature. Eur. J. Gen. Pract., 1996;2:65-70.

2. M. van den Akker, F. Buntinx, J.F. Metsemakers, S. Roos, and J.A. Knottnerus. Multimorbidity in general practice: prevalence, incidence, and determinants of co-occurring chronic and recurrent diseases. J. Clin. Epidemiol., 1998;51(5):367-375.

3. M. Fortin, C. Hudon, J. Haggerty, M. Akker, and J. Almirall. Prevalence estimates of multimorbidity: a comparative study of two sources. BMC Health Services Research, 2010;6(10).

4. S. Aarts, M. van den Akker, F.E. Tan, F.R. Verhey, J.F. Metsemakers, and M.P. van Boxtel. Influence of multimorbidity on cognition in a normal aging population: a 12-year follow-up in the Maastricht Aging Study. Int. J. Geriatr. Psychiatry, 2011;26(10):1046-1053.

5. L.G. Glynn, J.M. Valderas, P. Healy, E. Burke, J. Newell, P. Gillespie, and A.W. Murphy. The prevalence of multimorbidity in primary care and its effect on health care utilization and cost. Fam. Pract., 2011;28(5):516-523.

6. H. Galenkamp, A.W. Braam, M. Huisman, and D.J. Deeg. Somatic multimorbidity and self-rated health in the older population. J. Gerontol. B. Psychol. Sci. Soc. Sci., 2011;66(3):380-386.

7. L.H. Andrade, I.M. Bensenor, M.C. Viana, S. Andreoni, and Y.P. Wang. Clustering of psychiatric and somatic illnesses in the general population: multimorbidity and socioeconomic correlates. Braz. J. Med. Biol. Res., 2010;43(5):483-491.

8. E.B. Saltzer and E.I. Saltzer. Internal control and health. Which comes first?. West. J. Nurs. Res, 1987;9(4):542-554.

9. E. Loza, J.A. Jover, L. Rodriguez, and L. Carmona. Multimorbidity: Prevalence, Effect on Quality of Life and Daily Functioning, and Variation of This Effect When one Condition Is a Rheumatic Disease. Semin. Arthritis Rheum., 2008;38(4):312-349. .

10. J.F. Metsemakers, P. Hoppener, J.A. Knottnerus, R.J. Kocken, and C.B. Limonard. Computerized health information in The Netherlands: a registration network of family practices. Br. J. Gen. Pract., 1992;42(356):102-106.

11. C.c.o. WONCA. ICPC International Classification of Primary Care. 1987, Oxford: Oxford University Press.

12. J.F.M. Metsemakers, J.A. Knottnerus, G.J. van Schendel, R.J. Kocken, and C.B. Limonard. Unlocking patients' records in general practice for research, medical education and quality assurance: the Registration Network Family Practices. Int. J. Biomed. Comput., 1996;42(1-2):43-50.

13. J. Jolles, M.P. van Boxtel, R.W. Ponds, J.F. Metsemakers, and P.J. Houx. The Maastricht aging study (MAAS). The longitudinal perspective of cognitive aging. Tijdschrijft voor Gerontologie en Geriatrie, 1998;29(3):120-129.

14. J.E. Ware, Jr. and C.D. Sherbourne. The MOS 36-item short-form health survey (SF-36). I. Conceptual framework and item selection. Medical Care, 1992;30(6):473-483.

15. J.A. Knottnerus, J. Metsemakers, P. Hoppener, and C. Limonard. Chronic illness in the community and the concept of 'social prevalence'. Fam. Pract., 1992;9(1):15-21.

16. S.E. de Bie. Standaardvragen 1987: Voorstellen van uniformering van vraagstellingen naar achtergrondkenmerken en interviews. 2nd ed. 1987, Leiden, The Netherlands: Leiden University Press.

17. F.E.S. Tan. Best practices in analysis of longitudinal data: a multilevel approach, in Best practices in quantitative methods, Osborne J.W., Editor. 2008: London.451-471.

18. M. Rijken, M. van Kerkhof, J. Dekker, and F.G. Schellevis. Comorbidity of chronic diseases: effects of disease pairs on physical and mental functioning. Qual. Life Res. , 2005;14(1):45-55.

19. M. Fortin, G. Bravo, C. Hudon, L. Lapointe, J. Almirall, M.F. Dubois, and A. Vanasse. Relationship between multimorbidity and health-related quality of life of patients in primary care. Qual. Life Res., 2006;15(1):83-91. 
20. M. van den Akker, F. Buntinx, J.F. Metsemakers, S. Roos, and J.A. Knottnerus. Multimorbidity in general practice: prevalence, incidence, and determinants of co-occurring chronic and recurrent diseases. J. Clin. Epidemiol., 1998;51(5):367-375.

21. W.A. Arrindell, D.P. Barelds, I.C. Janssen, F.M. Buwalda, and J. van der Ende. Invariance of SCL-90$\mathrm{R}$ dimensions of symptom distress in patients with peri partum pelvic pain (PPPP) syndrome. Br. J. Clin. Psychol., 2006;45:377-391.

22. M. Fortin, C. Hudon, M.F. Dubois, J. Almirall, L. Lapointe, and H. Soubhi. Comparative assessment of three different indices of multimorbidity for studies on health-related quality of life. Health Qual. Life Outcomes, 2005;23(3):74.

23. L.G. Glynn, J.M. Valderas, P. Healy, E. Burke, J. Newell, P. Gillespie, and A.W. Murphy. The prevalence of multimorbidity in primary care and its effect on health care utilization and cost. Fam. Pract., 2011;28(5):516-523. 


\section{APPENDIX I}

Prevalence of (multi)morbidity groups on baseline, 3 and 6 year follow-up

\begin{tabular}{lllll}
\hline Group & Baseline & 3 yrs FU & 6 yrs FU & $\mathrm{N}$ \\
\hline 0 & Healthy & Healthy & Healthy & 338 \\
1 & Healthy & Healthy & Morbidity & 33 \\
2 & Healthy & Healthy & Multimorbidity & 17 \\
3 & Healthy & Morbidity & Morbidity & 29 \\
4 & Healthy & Morbidity & Multimorbidity & 11 \\
5 & Morbidity & Morbidity & Morbidity & 232 \\
6 & Morbidity & Morbidity & Multimorbidity & 43 \\
7 & Morbidity & Multimorbidity & Multimorbidity & 47 \\
8 & Healthy & Multimorbidity & Multimorbidity & 14 \\
9 & Multimorbidity & Multimorbidity & Multimorbidity & 420 \\
\hline
\end{tabular}

\section{APPENDIX II}

ICPC codes and the corresponding clusters

\begin{tabular}{ll}
\hline Cluster description & ICPC code \\
\hline All malignancies & A79, B72, B73, D74, D75, D76, D77, F74, N74, \\
& R84, R85, S77, T71, U75, U76, U77, W72, X75, \\
& X76, X77, Y77, Y78 \\
& D85, D86 \\
Peptic ulcers & D92, D93, D94, D97 \\
Other chronic gastro-intestinal diseases & F83, F84, F94 \\
Diseases of the eye & H83, H86, \\
Diseases of the ear & K74, K75, K76 \\
Ischemic diseases & K93, K94 \\
Pulmonary embolism and phlebitis & K89, K90, K91 \\
Cerebrovascular diseases & K78, K79, K80, K77, K82, K83 \\
Arrhythmias \& heart failure & K87, K92 \\
Other cardiovascular diseases & L84, L85, L88, L89, L90, L91, L95, L98 \\
Movement disorders & N87 \\
Parkinsonism & N89, N90 \\
Migraine \& Headache & N92, N70, N86, N88 \\
Other diseases of the nervous system & P76, P73 \\
Mood disorders & P70 \\
Alzheimer & P28, P71, P72, P74, P75, P77, P79, P80, P98 \\
Other mental disorders & R91, R95, R96, \\
Asthma, COPD and bronchitis & R70, R75, R97 \\
Other chronic respiratory diseases & S87, S91, S97 \\
Eczema, psoriasis and chronic skin ulcer & T85, T86, T92, T93, T99 \\
Endocrine diseases & T90 \\
Diabetes mellitus (Type I and II) & U04, U85, U88 \\
Diseases of the urinary tract &
\end{tabular}




\section{APPENDIX III}

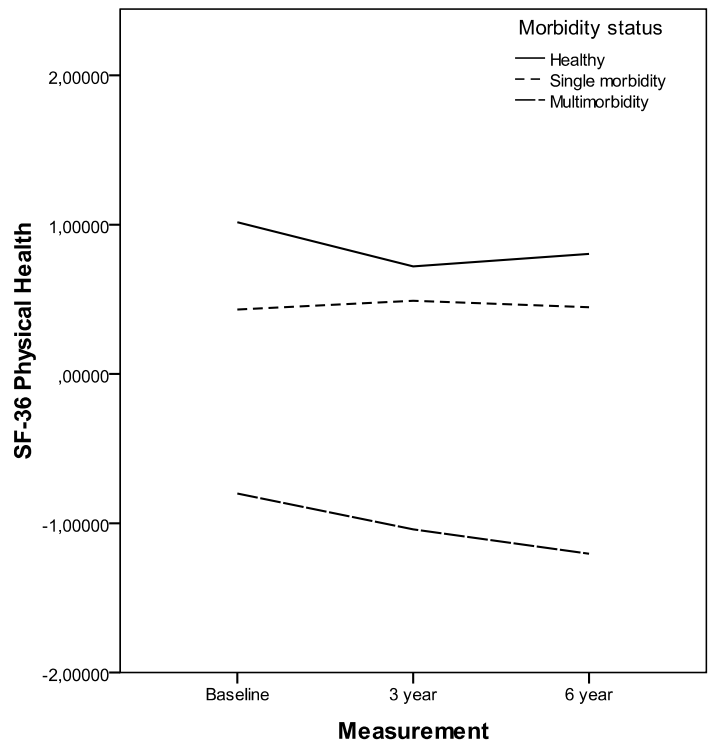

Figure 1.a. Age, sex and education adjusted z-scores (with mean= 0 and standard deviation=1) for physical health per morbidity status (i.e. healthy vs. single morbidity and healthy vs. multimorbidity)

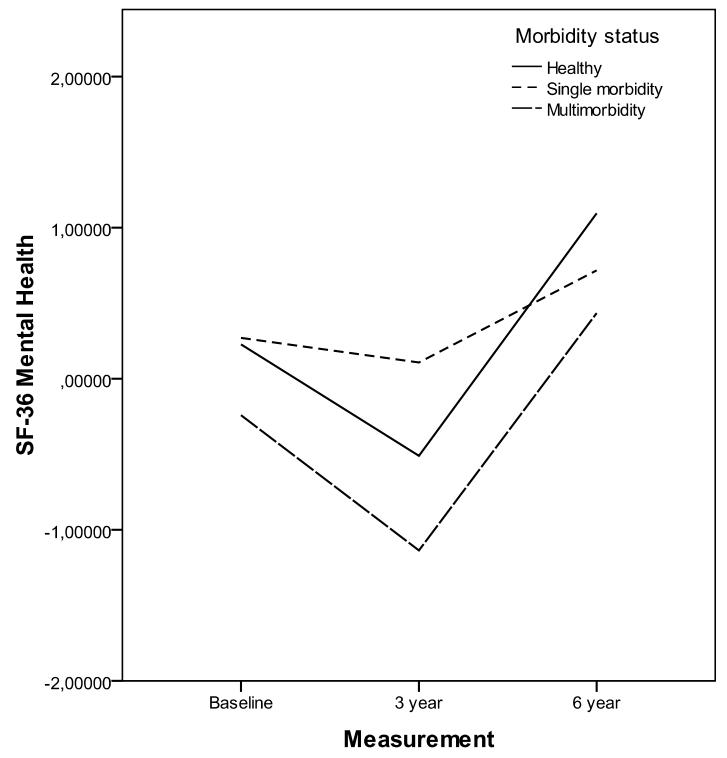

Figure 1.b. Age, sex and education adjusted $z$-scores (with mean $=0$ and standard deviation=1) for mental health per morbidity status (i.e. healthy vs. single morbidity and healthy vs. multimorbidity). 
The effect of multimorbidity on health related functioning: temporary or persistent?

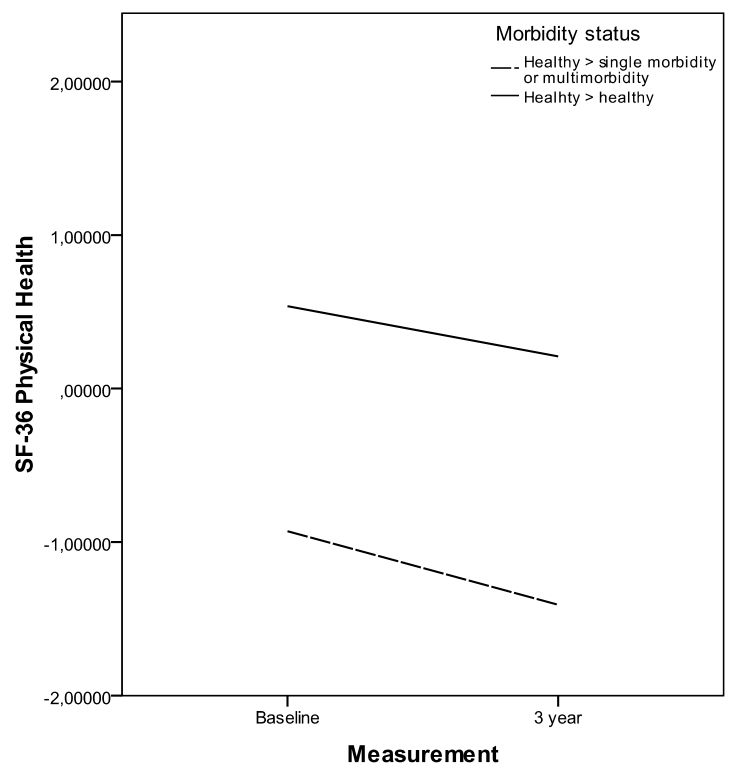

Figure 2.a. Age, sex and education adjusted z-scores (with mean= 0 and standard deviation=1) for physical health per morbidity status (i.e. no change in disease status (healthy $>$ healthy) vs. a change in disease status (healthy $>$ single morbidity or multimorbidity).

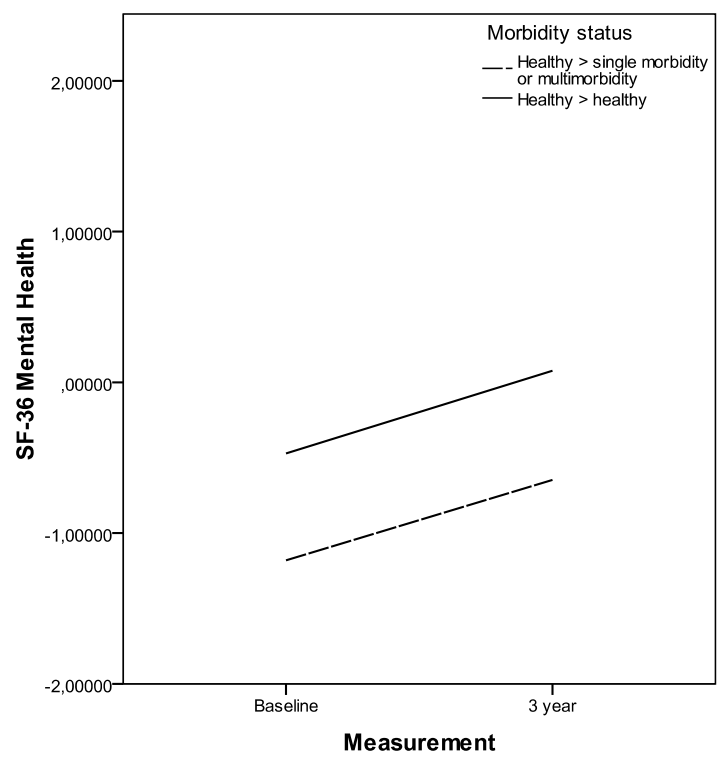

Figure 2.b. Age, sex and education adjusted $z$-scores (with mean $=0$ and standard deviation=1) in mental health, i.e. no change in disease status (healthy > healthy) vs. a change in disease status (healthy $>$ single morbidity or multimorbidity). 


\section{APPENDIX IV}

Table 4. Multilevel analyses for the association between single morbidity and multimorbidity on one side and depressive and anxiety complaints (SCL-90) on the other, adjusted for age, gender and education

\begin{tabular}{|c|c|c|c|c|}
\hline & \multicolumn{2}{|c|}{ Depression } & \multicolumn{2}{|c|}{ Anxiety } \\
\hline & $\begin{array}{c}\text { Parameter } \\
\text { estimate }\end{array}$ & P-value & $\begin{array}{l}\text { Parameter } \\
\text { estimate }\end{array}$ & P-value \\
\hline Age & 0.017 & 0.352 & 0.011 & 0.332 \\
\hline Gender & 1.104 & 0.021 & 0.364 & 0.198 \\
\hline Education & -1.222 & $<0.001$ & -0.730 & $<0.001$ \\
\hline \multicolumn{5}{|l|}{ Single morbidity } \\
\hline Baseline & 1.198 & 0.286 & 1.155 & 0.115 \\
\hline 3 year follow-up & -0.650 & 0.419 & -0.235 & 0.625 \\
\hline 6 year follow-up & 0.066 & 0.939 & -0.018 & 0.970 \\
\hline \multicolumn{5}{|l|}{ Multimorbidity } \\
\hline Baseline & 3.164 & 0.002 & 2.236 & 0.001 \\
\hline 3 year follow-up & 1.120 & 0.138 & 1.310 & 0.004 \\
\hline 6 year follow-up & 0.638 & 0.447 & 0.219 & 0.632 \\
\hline \multicolumn{5}{|l|}{ Interaction } \\
\hline $\begin{array}{l}\text { Single morbidity* time } \\
\text { (baseline and } 3 \text { year follow-up) }\end{array}$ & -1.850 & 0.142 & -1.390 & 0.090 \\
\hline $\begin{array}{l}\text { Single morbidity }{ }^{*} \text { time } \\
(3 \text { year FU and } 6 \text { year follow-up) }\end{array}$ & 0.718 & 0.470 & -0.217 & 0.686 \\
\hline $\begin{array}{l}\text { Multimorbidity * time } \\
\text { (baseline and } 3 \text { year follow-up) }\end{array}$ & -2.041 & 0.059 & -0.926 & 0.186 \\
\hline $\begin{array}{l}\text { Multimorbidity * time } \\
(3 \text { year FU and } 6 \text { year follow-up) }\end{array}$ & -0.485 & 0.586 & 1.092 & 0.023 \\
\hline
\end{tabular}

Note: the reference group consisted of healthy participants 
The effect of multimorbidity on health related functioning: temporary or persistent?

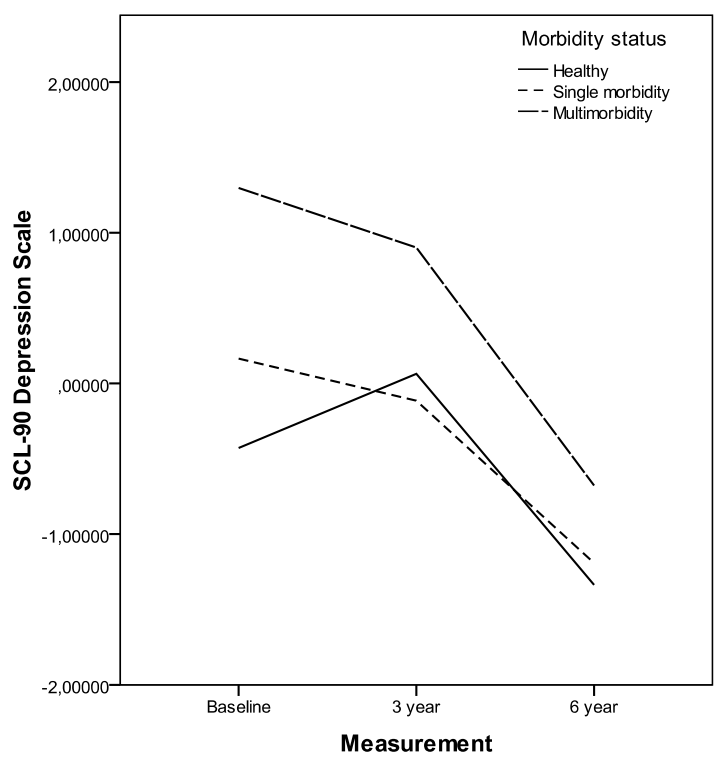

Figure 3.a. Age, sex and education adjusted z-scores (with mean= 0 and standard deviation=1) for depressive complaints per morbidity status (i.e. healthy vs. single morbidity and healthy vs. multimorbidity).

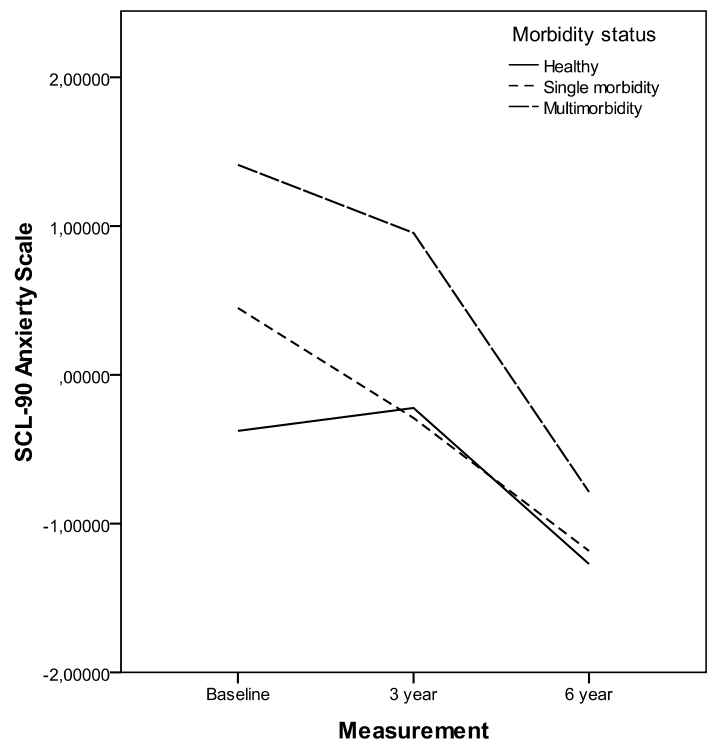

Figure 3.b. Age, sex and education adjusted z-scores (with mean $=0$ and standard deviation=1) for anxiety complaints per morbidity status (i.e. healthy vs. single morbidity and healthy vs. multimorbidity). 



\section{CHAPTER 5}

Co-presence of disability and multimorbidity with frailty: an examination of heterogeneity in the frail older population

Under review as:

S. Aarts, K.V. Patel, M. E. Garcia, M. van den Akker, F.R.J. Verhey, J.F.M. Metsemakers, M.P.J. van Boxtel, V. Gudnason, M.K. Jonsdottir, K. Siggeirsdottir, P.V. Jonsson, T. B. Harris and L.J. Launer. 


\section{Chapter 5}

\section{ABSTRACT}

\section{Background}

Frailty is often associated with multimorbidity and disability. We investigated heterogeneity in the frail older population by characterizing five subpopulations according to quantitative biological markers, multimorbidity and disability, and examined their association with mortality and nursing home admission.

\section{Methods}

Participants ( $n=4,414$, mean age 76.4; SD: 5.5) were from the population-based Age Gene/Environment Susceptibility Reykjavik Study. Frailty was defined by $\geq 3$ of the following five characteristics: weight loss, weakness, reduced energy levels, slowness and physical inactivity. Multimorbidity, the co-occurrence of two or more chronic medical conditions, was assessed using a simple disease count, based on 13 prevalent conditions. Disability was assessed by five activities of daily living; participants who had difficulty with one or more tasks were considered disabled. Differences among frail subpopulations were based on the co-presence of multimorbidity and disability. Differences among the following subpopulations were examined: 1) Non-frail (reference group); 2) Frail only; 3) Frail with disability; 4) Frailty with multimorbidity; 5) Frail with disability and multimorbidity.

\section{Results}

Frailty was present in $10.7 \%(n=473)$. Although frailty was associated with increased risk for mortality (OR 1.40; 95\% CI 1.16-1.70) and nursing home admission (OR 1.17; 95\% 1.15-2.10), risks differed by subpopulations. Compared to the non-frail, the frail only group had poorer cognition and increased inflammation levels but did not have increased risk for mortality (OR 1.36; 95\% 0.82-2.25), or nursing home admission (OR 1.03; 95\% 0.48-2.23). Compared to the non-frail, the other three frail subpopulations had significantly poorer cognition, increased inflammation levels, more white matter lesions, higher levels of calcium, glucose and red cell distribution width and increased risk for mortality and nursing home admission.

\section{Conclusions}

The adverse health risks associated with frailty in the general older adult population may primarily be driven by increased disease burden and disability. 


\section{INTRODUCTION}

Frailty is described as a state of increased vulnerability that may result from decreased physiological reserve, multi-system dysregulation and diminished capacity to maintain homeostasis1. Although the prevalence of frailty depends on the case definition and source population, all studies demonstrate that frailty increases with advancing age2. For example, the overall prevalence of frailty among 5,317 community-dwelling older adults participating in the Cardiovascular Health Study was 6.9\%, but in participants who were 80 and older the prevalence was 30\%2. Frailty increases the risk for a variety of adverse health outcomes including hospitalization3, disability 4 and mortality 5, 6, which may exert a high burden on health care systems.

Attempts to study frailty are complicated by the lack of consensus on the operational definition of frailty ${ }^{7}$; the term frailty is often used interchangeably with other geriatric conditions, such as multimorbidity and disability. However, previous research suggests that frailty not only exists independent of age-associated diseases, but could also contribute to the incidence and progression of multimorbidity and disability ${ }^{2}$. Moreover, frailty and multimorbidity are both known to be independent risk factors for disability. Disability in turn, may exacerbate the health related problems associated with frailty and multimorbidity. Hence, the three conditions are interrelated but the degree of mutual dependence is not well established. While previous studies have examined the effect of frailty on mortality and nursing home admission adjusting for the presence of disability and multimorbidity, no studies have characterized the co-occurrence of frailty with disability and multimorbidity in relation to health outcomes. Therefore, it remains unclear whether the risks for adverse outcomes associated with frailty are driven by disease burden ${ }^{8}$, including possibly subclinical diseases and impairments that may underlie frailty ${ }^{8}$.

Given the correlation between frailty, multimorbidity and disability, we sought to investigate heterogeneity within the frail population in a large cohort of communitydwelling older adults. . The first aim was to estimate the overall prevalence of frailty in community dwelling older adults as well as to estimate the co-presence of disability and multimorbidity within the frail population. The second aim was to characterize subpopulations of frail older adults with quantitative physiological markers. Since the risk of adverse outcomes may differ per subpopulation of frailty, the last aim was to examine the risk for mortality and nursing home admission in subpopulations of frail and non-frail older adults.

\section{METHODS}

\section{Study Population}

The Age Gene/Environment Susceptibility-Reykjavik (AGES-Reykjavik) Study is based on the Reykjavik Study, a population-based cohort established in 1967 by investigators at the Icelandic Heart Association (IHA) ${ }^{9}{ }^{10}$. This cohort originally comprised a random sample of men and women born between 1907 and 1935 and living in Reykjavik at baseline. The initial follow-up examination for AGES-Reykjavik started in 2002 and was completed in $2006^{11}$. As part of the AGES-Reykjavik Study, participants $(n=5,764)$ completed interviews 
about a variety of health related factors, including functional status, medical conditions, health behaviours, and family history. Moreover, participants had a series of standardized health examinations. AGES-Reykjavik was approved by the National Bioethics Committee in Iceland that acts as the institutional review board for the IHA and by the National Institute on Aging's Institutional Review Board. Release of data for analysis is governed by rules created by these bodies to protect the privacy of Icelandic participants ${ }^{11}$. Participants with Parkinson's disease (i.e. their symptoms and functional impairments would interfere significantly with the frailty assessment), participants who had not undertaken an MRI scan or had missing data on the variables on which the subpopulations of the current study were based (i.e. frailty characteristics, multimorbidity and disability) were excluded ( $n=1,350$; 23.4\%). People who were excluded were older and less educated than those included in the analysis. There was no difference in the sex distribution. The final analytic study sample yielded 4,414 participants.

\section{Assessment of Frailty}

The present study used the operational definition of frailty developed by Fried and colleagues $^{6,12}$. The phenotype of frailty was identified by the presence of three or more of the following characteristics ${ }^{6}$ :

(1) Weight loss: unintentional weight loss of $5 \mathrm{~kg}$ or more in the past 12 months or a Body Mass Index (BMI) less than 18.5.

(2) Weakness: hand grip strength in the lowest $20 \%$ adjusted for height and sex.

(3) Low energy: reduced energy level determined by the respondent answering 'no' to 'Do you feel full of energy?' in the Geriatric Depression Scale.

(4) Slowness: slowest $20 \%$ of the study population, based on usual gait speed over a 6 meter course, adjusted for height and sex.

(5) Low physical activity: no swimming or walking during the summer and winter season

and no participation in low and moderate/vigorous physical activity during the last 12 months.

\section{Assessment of Disability}

Difficulty in carrying out activities of daily living (i.e. walking from one room to another, getting in and out of bed, eating, dressing and showering/bathing) were assessed by five questions ${ }^{13}$. Each question had a 4-point Likert response: 1) 'no difficulty', 2) 'some difficulty', 3) 'much difficulty', and 4) 'I am unable to do it'. Participants who had difficulty with at least one ADL task (i.e. a score of 2 or more on one of the five questions) were considered disabled.

\section{Assessment of Multimorbidity}

Multimorbidity was defined as the co-occurrence of two or more diseases ${ }^{14}$ and was quantified by counting the number of diseases ${ }^{15}$ (see Appendix). Anemia was defined based on the World Health Organization criteria of hemoglobin concentration of $<12 \mathrm{~g} / \mathrm{dL}$ in women and $<13 \mathrm{~g} / \mathrm{dL}$ in men. CKD was defined by using an estimated glomerular filtration 
rate of less than $60 \mathrm{~mL} / \mathrm{min} / 1.73 \mathrm{~m}^{2}$ calculated by the Modification of Diet in Renal Disease $(\mathrm{MDRD})^{16}$. Diabetes was defined as a history of diabetes, use of glucose-modifying medication, or fasting blood glucose of $>7 \mathrm{mmol} / \mathrm{L}$. CHD and angina pectoris were both based on electrocardiograms and questionnaire ${ }^{17}$.

To assess cognitive status and diagnose dementia cases, participants with an MMSE score of $\leq 24^{18}$ and a DDST score of $\leq 17^{19}$, were administered a diagnostic battery of neuropsychological tests and were examined by a neurologist. A consensus diagnosis of dementia, according to international guidelines, was made by a panel that included a geriatrician, neurologist, neuropsychologist and neuroradiologist. The remaining medical conditions were based on the AGES health questionnaire. All questions related to these medical conditions were formulated in the same manner (e.g. 'Has a doctor or other health professional ever told you that you had cancer?'). The answer 'I don't know' was assigned a missing value.

\section{Frailty Groups}

The following four subpopulations of participants with frailty were examined: 1) 'Only frail' (no disability or multimorbidity); 2), 'Frail with disability' (FD; no multimorbidity); 3) 'Frail with multimorbidity' (FM; no disability), and 4) 'Frail with disability and multimorbidity (FDM). For all analyses, the non-frail participants, who did not meet frailty criteria, served as the reference group.

\section{Assessment of Markers of Physiological Dysfunction}

The following quantitative markers which are known to reflect disease burden and/or subclinical impairment were examined: White matter hyperintensities (WMH) were assessed by MRI and divided by total intracranial volume,. The presence of brain infarcts was identified as defects in the brain parenchyma with hyperintensities on T2 and FLAIR images with a maximal diameter of at least $4 \mathrm{~mm}^{20}$. Glucose levels were determined by a fasting morning blood draw. Coronary artery calcium level (CAC) was quantified by summing all 4 coronary arteries according to the Agatston method 20, 21. Estimated Glomerular Filtration Rate (eGFR) was calculated using the Modification of Diet in Renal Disease (MDRD) formula ${ }^{16}$. The red cell distribution width (RDW) was reported as part of the complete blood count ${ }^{22}$. C-Reactive Protein (CRP) was measured on a Hitachi 912, using reagents from Roche Diagnostics ${ }^{23}$. Bone Mineral Density was assessed by the amount of bone matter per cubic centimeter ${ }^{24}$. Composite scores for speed of processing, memory and executive functioning were constructed (details have been described elsewhere ${ }^{25}$ ).

\section{Assessment of Mortality and Nursing Home Admission}

Every participant was followed until 31, May 2010 for mortality and nursing home admission. Mean follow-up was 5.94 (SD: 1.22) year for nursing home admission and 7.76 (SD: 1.44) for mortality. Mortality information is provided by Statistics Iceland 
(www.statice.is). Information on nursing home admission stems from the Inter Resident Assessment Instrument (InterRAI) ${ }^{26}$.

\section{Statistical Analysis}

Linear and logistic regression analyses, adjusted for age and sex, were used to determine differences in quantitative disease markers by subpopulations of frailty. Cox proportional hazards models were used to determine the association of frailty status with mortality and incident nursing home admission.

The first model was adjusted for age and sex only, while the second model was adjusted for age at time of examination, sex, education (high vs. low education; primary and secondary school vs. college and university), smoking status (never smoked, former smoker and current smoker), alcohol intake (number of glasses per week), living arrangement (living alone vs. living together), depressive symptoms (assessed by the 15-item Geriatric Depression Scale), disability, all included diseases and total disease count. Hazard ratios (HR) and 95\% confidence intervals (CI) are reported. P-values of 0.05 or less were considered statistically significant. Statistical analyses were conducted using the SPSS statistical software package version 18.0 for Windows (SPSS Inc., Chicago, IL, USA).

\section{RESULTS}

Figure 1 illustrates the co-occurrence of frailty, disability and multimorbidity. The overall prevalence of frailty was $10.7 \%(n=473)$. In total, $4.3 \%(n=190)$ of older adults had frailty, disability and multimorbidity, while $2.1 \%(\mathrm{n}=94)$ were frail and disabled and $2.8 \%(\mathrm{n}=$ $125)$ had frailty and multimorbidity. Among the 473 frail participants, 40.2\% ( $n=190)$ had disability and multimorbidity. Although the majority of frail participants reported ADL disability and/or multimorbidity, $13.5 \%(n=64)$ did not report ADL disability and did not have any of the condition used to assess multimorbidity (i.e. 'only frail'). Descriptive characteristics of the 4,414 participants are shown in Table 1. The average age was 76.4 years and $57.4 \%(n=2532)$ were female.

Frail individuals were older, less educated, more likely to be living alone and reported more depressive symptoms than non-frail participants. In general, frailty characteristics were similarly distributed across the frail subpopulations; however, the weight loss criterion was more common in the 'only frail' group compared with the other frail subpopulations (Table 2).

Frail older adults were approximately twice more likely to die or be admitted to a nursing home compared with non-frail participants, adjusting for age and sex (Model 1, Table 4). The effect of frailty on each outcome remained significant after further adjusting for individual diseases, total disease count, and disability although the effect sizes were attenuated (Model 2). 


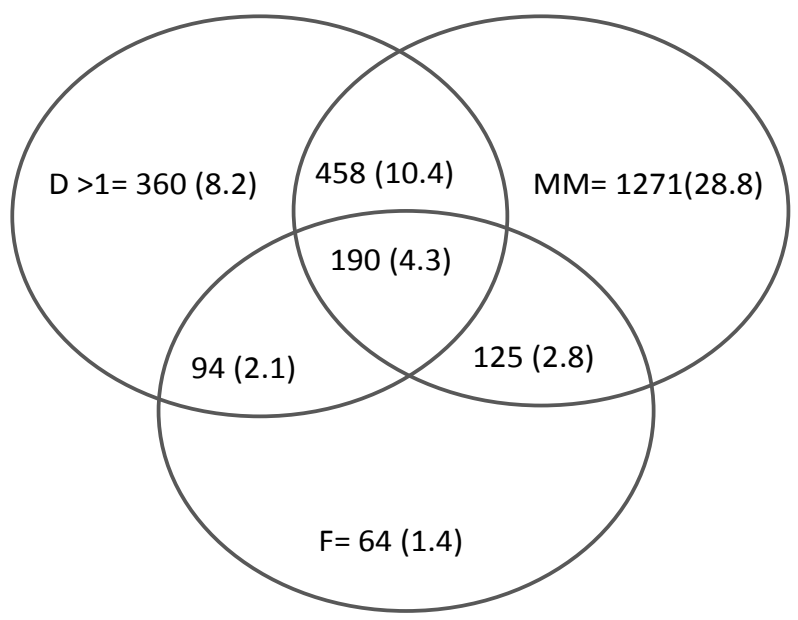

Figure 1. Venn diagram displaying the overlap in prevalence (\%) between frailty, multimorbidity and disability. $F=$ Only frail , $D=$ Disability, $M M=$ Multimorbidity. Note: total $n=4414$; healthy $\mathrm{n}=1852$ (42.0\%; i.e. no frailty, multimorbidity or disability).

After adjusting for age and sex, several quantitative markers of disease burden and functional impairment were significantly associated with 'only frail' status (Table 3). Participants who were 'only frail' had decreased memory function, processing speed and executive function and had higher CRP values compared with non-frail participants. Interestingly, however, there was no difference between these two groups in terms of white matter lesions and brain infarcts as well as no differences in BMD, CAC, eGFR, glucose level and RDW. The other three frail subpopulations (FD, FM and FDM) had poorer neurocognitive performance than the non-frail group, but also higher white matter lesions, CAC, and RDW values. No differences in BMD or eGFR were observed in these groups.

Table 5 shows the relative risks for mortality and nursing home admission according to frail subpopulations. Compared with non-frail older adults, frail participants with multimorbidity (FM and FDM subpopulations) had a significantly increased risk for mortality; however, those who were 'only frail' and those with frailty and disability (FD) did not have an increased mortality risk. In addition, the 'only frail' subpopulation without disability and multimorbidity did not have an increased risk for nursing home admission compared with non-frail participants. In contrast, frail participants with disability and frail participants with frailty, disability and multimorbidity were significantly more likely to be admitted to a nursing home compared with non-frail older participants 


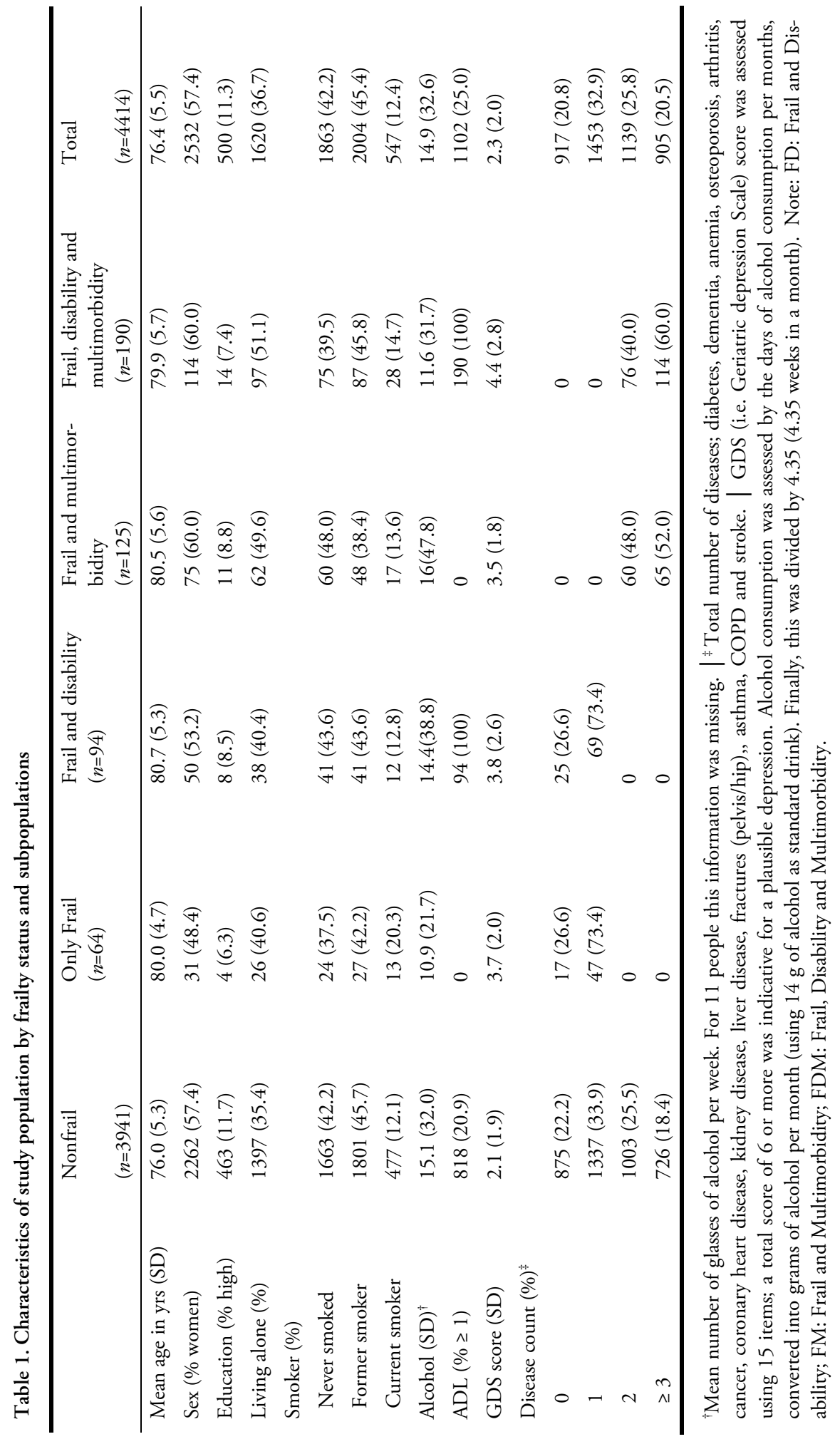


Table 2. Prevalence of frailty characteristics

\begin{tabular}{|c|c|c|c|c|c|c|}
\hline & $\begin{array}{l}\text { Nonfrail } \\
(n=3941)\end{array}$ & $\begin{array}{l}\text { Only } \\
\text { Frail } \\
(\mathrm{n}=64)\end{array}$ & $\begin{array}{l}\text { Frail and } \\
\text { disability } \\
(n=94)\end{array}$ & $\begin{array}{c}\text { Frail and } \\
\text { multimorbidity } \\
(\mathrm{n}=125)\end{array}$ & $\begin{array}{c}\text { Frail and } \\
\text { disability and } \\
\text { multimorbidity } \\
(\mathrm{n}=190)\end{array}$ & $(\mathrm{n}=4414)$ \\
\hline Low energy & $\begin{array}{c}1798 \\
(45.6)\end{array}$ & 62 (96.9) & $\begin{array}{c}89 \\
(94.7)\end{array}$ & $\begin{array}{c}121 \\
(96.8)\end{array}$ & $\begin{array}{c}184 \\
(97.0)\end{array}$ & $\begin{array}{c}2254 \\
(51.1)\end{array}$ \\
\hline $\begin{array}{l}\text { Low physical } \\
\text { activity }\end{array}$ & $\begin{array}{l}345 \\
(8.8)\end{array}$ & $32(50.0)$ & $\begin{array}{c}55 \\
(58.5)\end{array}$ & $\begin{array}{c}56 \\
(44.8)\end{array}$ & $\begin{array}{c}107 \\
(56.3)\end{array}$ & $\begin{array}{c}595 \\
(13.5)\end{array}$ \\
\hline Weakness & $\begin{array}{c}512 \\
(13.0)\end{array}$ & $46(71.9)$ & $\begin{array}{c}64 \\
(68.1)\end{array}$ & $\begin{array}{c}95 \\
(76.0)\end{array}$ & $\begin{array}{c}125 \\
(65.8)\end{array}$ & $\begin{array}{c}842 \\
(19.1)\end{array}$ \\
\hline Weight loss & $\begin{array}{l}127 \\
(3.2)\end{array}$ & $20(31.3)$ & $\begin{array}{c}12 \\
(12.8)\end{array}$ & $\begin{array}{c}27 \\
(21.6)\end{array}$ & $\begin{array}{c}37 \\
(19.5)\end{array}$ & $\begin{array}{l}223 \\
(5.1)\end{array}$ \\
\hline Slowness & $\begin{array}{c}435 \\
(11.0)\end{array}$ & $44(68.8)$ & $\begin{array}{c}79 \\
(84.0)\end{array}$ & $\begin{array}{c}95 \\
(76.0)\end{array}$ & $\begin{array}{c}167 \\
(87.9)\end{array}$ & $\begin{array}{c}820 \\
(18.0)\end{array}$ \\
\hline Mean $(\mathrm{SD}) \S$ & $\begin{array}{c}0.82 \\
(0.74)\end{array}$ & $\begin{array}{c}3.19 \\
(0.39)\end{array}$ & $\begin{array}{l}3.18 \\
(0.39)\end{array}$ & $\begin{array}{c}3.15 \\
(0.38) \\
\end{array}$ & $\begin{array}{c}3.26 \\
(0.48) \\
\end{array}$ & $\begin{array}{c}1.07 \\
(1.03) \\
\end{array}$ \\
\hline
\end{tabular}

$\$$ Mean number of frailty characteristics. Low energy: reduced energy level determined by the respondent answering 'no' to 'Do you feel full of energy?' in the Geriatric Depression Scale; Low physical activity: based on questionnaire, respondents who report not swimming or walking during the summer and winter season and not participating in low and moderate/vigorous physical activity during the last 12 months; Weakness: hand grip strength in the lowest $20 \%$ adjusted for height and sex; Weight loss: unintentional weight loss of $5 \mathrm{~kg}$ or more in the past 12 months or a Body Mass Index (BMI) less than 18.5; Slowness: slowest $20 \%$ of the study population, based on usual gait speed over a 6 -meter course, adjusted for height and sex.

Table 4. Risk of death and nursing home admission according to frailty status

\begin{tabular}{|c|c|c|c|c|}
\hline & $\begin{array}{l}\text { \# events } \\
\text { (\% within } \\
\text { group) }\end{array}$ & $\begin{array}{c}\text { Event rate per } \\
1000 \\
\text { person years }\end{array}$ & $\begin{array}{c}\text { Model } 1 \\
\text { HR }(95 \% \text { CI) }\end{array}$ & $\begin{array}{c}\text { Model } 2 \\
\text { HR }(95 \% \text { CI })\end{array}$ \\
\hline \multicolumn{5}{|l|}{ Mortality } \\
\hline Nonfrail & $656(16.6)$ & 29 & 1.00 & 1.00 \\
\hline Frail & $191(40.3)$ & 79 & $1.86(1.57-2.21)$ & $1.40(1.16-1.70)$ \\
\hline \multicolumn{5}{|c|}{ Nursing home admission } \\
\hline Nonfrail & $280(7.1)$ & 12 & 1.00 & 1.00 \\
\hline Frail & $123(26.5)$ & 48 & $2.36(1.89-2.95)$ & $1.17(1.15-1.20)$ \\
\hline
\end{tabular}

Note: The reference groups consist of non-frail participants. HR: Hazard Ratio; CI: Confidence Interval.

Model 1: adjusted for age and sex; Model 2: adjusted for age, sex, education, smoking status, alcohol intake, living arrangements, disability, depressive symptoms, all included diseases and disease count. 


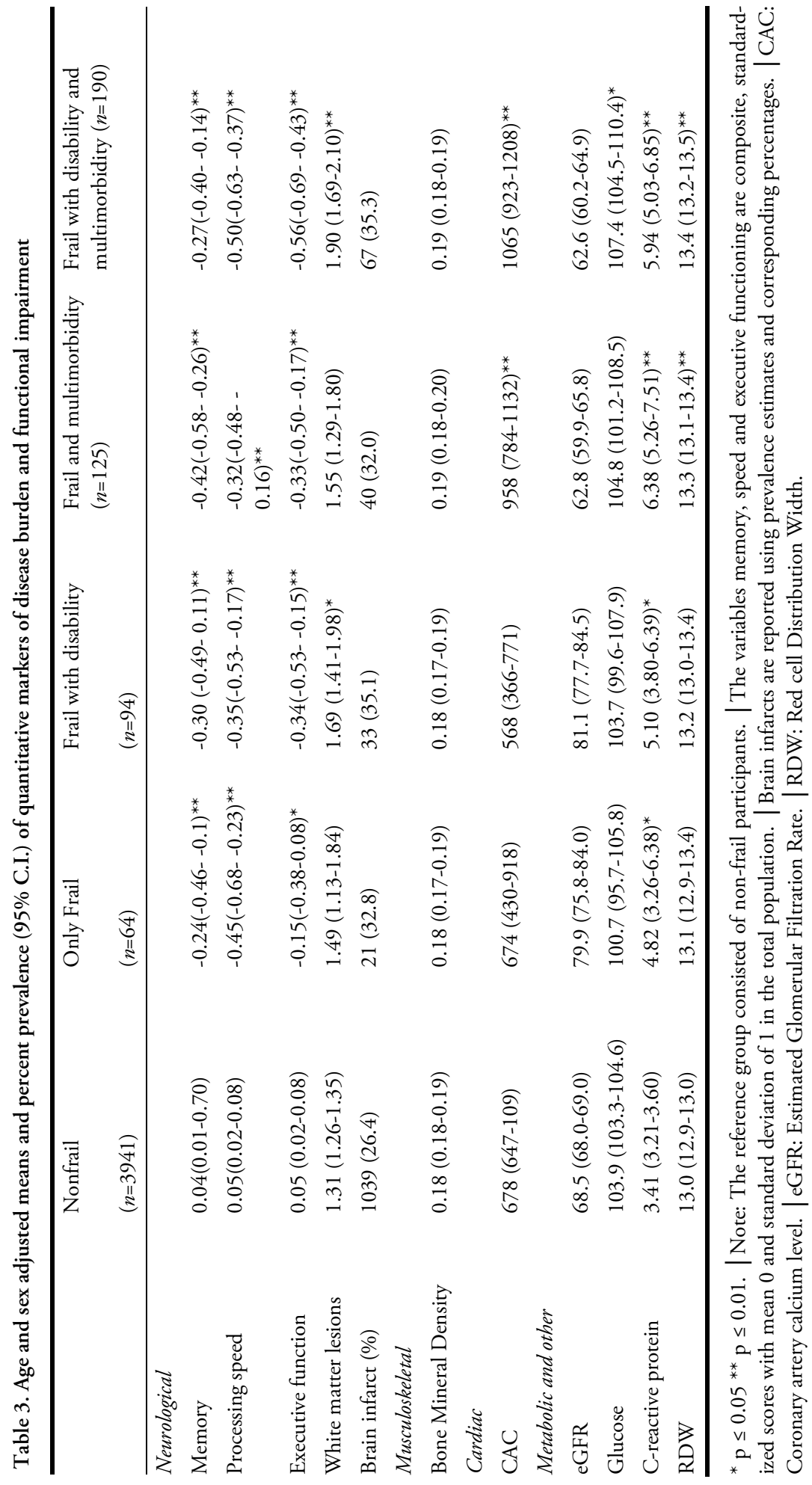


Table 5. Risk of death and nursing home admission in frail subpopulations

\begin{tabular}{|c|c|c|c|c|}
\hline & $\begin{array}{c}\text { \# events } \\
\text { (\% within group) }\end{array}$ & $\begin{array}{l}\text { Event rate per } \\
1000 \\
\text { person years }\end{array}$ & $\begin{array}{c}\text { Model } 1 \\
\text { HR }(95 \% \text { CI) }\end{array}$ & $\begin{array}{c}\text { Model } 2 \\
\text { HR }(95 \% \text { CI })\end{array}$ \\
\hline \multicolumn{5}{|l|}{ Mortality } \\
\hline Nonfrail & $656(16.6)$ & 29 & 1.00 & 1.00 \\
\hline Only frail & $18(28.1)$ & 52 & $1.24(0.78-1.99)$ & $1.36(0.82-2.25)$ \\
\hline FD & $27(48.7)$ & 54 & $1.14(0.78-1.69)$ & $1.17(0.74-1.86)$ \\
\hline FM & $56(44.8)$ & 90 & $2.10(1.59-2.78)$ & $1.50(1.11-2.02)$ \\
\hline FDM & $89(46.8)$ & 94 & $2.37(1.89-2.98)$ & $1.43(1.10-1.85)$ \\
\hline \multicolumn{5}{|c|}{ Nursing home admission } \\
\hline Nonfrail & $280(7.1)$ & 12 & 1.00 & 1.00 \\
\hline Only frail & $8(12.9)$ & 22 & $1.13(0.56-2.29)$ & $1.03(0.48-2.23)$ \\
\hline $\mathrm{FD}$ & $28(30.4)$ & 59 & $2.80(1.91-4.20)$ & $2.03(1.23-3.34)$ \\
\hline FM & $32(26.4)$ & 46 & $2.10(1.47-3.10)$ & $1.46(0.98-2.19)$ \\
\hline FDM & $54(28.7)$ & 53 & $2.65(1.96-3.58)$ & $1.44(1.02-2.03)$ \\
\hline $\begin{array}{l}\text { Note: The ref } \\
\text { Multimorbidit } \\
\text { Model 1: adj }\end{array}$ & $\begin{array}{l}\text { groups consist of } \\
\text { I: Frail, Disability } \\
\text { or age and sex; }\end{array}$ & $\begin{array}{l}\text { frail participa } \\
\text { Multimorbidit } \\
\text { 2: adjusted } \mathrm{f}\end{array}$ & \multicolumn{2}{|c|}{$\begin{array}{l}\text { FD: Frail and disability; FM: Frail and } \\
\text { R: Hazard Ratio; CI: Confidence Interval. } \\
\text { e, sex, education, smoking status, alcohol } \\
\text { cluded diseases and disease count. }\end{array}$} \\
\hline
\end{tabular}

\section{DISCUSSION}

The present study, conducted in a large population-based sample of older adults, sought to investigate the heterogeneity within the frail population by examining subpopulations of frailty. The present study shows that, although frailty is often accompanied by multimorbidity and disability, $13.5 \%$ of all frail people appeared to be 'only frail' (i.e. frail without the presence of multimorbidity and/or disability). Apart from weight loss, which was more apparent in the 'only frail' group, in general, the frailty characteristics were evenly distributed across the frail subpopulations. Compared with non-frail participants, the 'only frail' subgroup had significantly lower cognitive function and higher levels of CRP Our findings not only show that frailty can exist without the presence of multimorbidity and disability but also suggest, in congruence with previous research ${ }^{2}$, that frailty can result from age related physiologic changes that are not disease or disability based. The other three subpopulations of frailty not only showed a decrease in neurocognitive performance and elevated inflammation values, but were also associated with higher levels of white matter lesions (the FD and FDM group), CAC (i.e. the FM and FDM group) and RDW values (i.e. the FM and FDM group). In addition, the 'only frail' group was not at an increased risk of mortality and nursing home admission. While the FM and FDM group had an increased risk for mortality; the FD and FDM group had an increased risk for nursing home admission.

The present results indicate that although frailty is often accompanied by multimorbidity and disability, a substantial portion of older individuals are frail without having multimorbidity and/or disability. Our results are in congruence with a study by Fried and colleagues which claimed that frailty is not synonymous with either the presence of chronic diseases or disability ${ }^{2}$. A study by Fried and colleagues showed that $27 \%$ of the 
participants were frail without having comorbid diseases and ADL disability, while $21.5 \%$ of participants were frail with multimorbidity and ADL disability ${ }^{6}$. In the present study these percentages were respectively 13.5 and 40.2. The dissimilarity in prevalence estimates between the present study and the study conducted by Fried and colleagues could be due to differences regarding the assessment of frailty, ADL disability and multimorbidity. For example, while the study by Fried and colleagues used a more elaborate index to assess ADL disability, our study used a more complete nosological spectrum to assess multimorbidity. Moreover, the abovementioned difference in obtained prevalence estimates may also be explained by the difference in populations under study (i.e. Icelandic population and Americans from European and African descent)

Cognitive functioning, as assessed by memory function, processing speed and executive function, was significantly decreased in all frail subpopulations as compared with non-frail participants. White matter hyperintensities were only related subpopulations of frailty in which ADL disability was present (i.e. FD and FDM group). Previous evidence suggest that frailty is not only associated with cognitive decline but also with Alzheimer pathology on postmortem examination ${ }^{27}$. The mechanism through which neurocognitive dysfunction plays a role in frailty has not yet been defined. It is speculated that accumulation of Alzheimer pathology could affect components of frailty by impairing neural systems involved in planning and monitoring physical functioning ${ }^{27}$. Compared to the non-frail, the presence of brain infarcts was much higher in all subpopulations of frailty. Nevertheless, no significant association between brain infarcts and frail subgroups were detected. A study by Newman, based on the Cardiovascular Health study, illustrated that there is significant association between frailty and cerebral infarcts ${ }^{8}$. This difference in obtained effects might be due to the fact that the present study investigated subpopulation of frailty while Newman and colleagues investigated different stages of frailty (i.e. nonfrail, intermediate frail and frail).

Interestingly, the frail subpopulations with ADL disability (i.e. the FD and FDM group) showed elevated CAC levels. Although frailty has been associated with cardiovascular disease related outcomes ${ }^{8}$, the present results indicate that elevated CAC levels are not associated with frailty per se, but merely with the co-presence of ADL disability. The present study also provides evidence for the relation between inflammation and frailty, since elevated CRP levels appear to be present in all frail subpopulations. Recent evidence suggests that frailty is not related to one deficient dominant deregulated system (e.g. inflammation) but is associated with the total number of abnormal physiological systems ${ }^{28}$. Consequently, frailty has been suggested to be a nonlinear complex system that is independent of any specific system abnormalities ${ }^{28}$. Our results provide evidence for this postulation since various quantitative markers, such as CRP, RDW and fasting glucose ${ }^{29}$ all appear to be associated with frailty. The mechanism by which these impairments influence frailty still remains to be determined.

When evaluating the risk of mortality and nursing home admission for different frail subpopulations, our results indicate that, while mortality is primarily driven by disease burden, functional limitations seem to be the driving force behind nursing home 
admission. Hence, the absence of multimorbidity and disability in the 'only frail' lowers their risk for mortality and nursing home admission.

The current study does have some limitations that should be considered. Several medical conditions and disability were based on participants' self-reports, which could have compromised the validity of these data. Although self-reported disease status has been shown to correlate reasonably well with medical records, the oldest old (i.e. people aged 90 and over) tend to under-report certain diagnosed medical conditions ${ }^{30}$. Moreover, the present study included thirteen highly prevalent medical conditions, all well known for their profound negative effects on health. Nevertheless, other medical conditions that were not evaluated could not only have underestimated the prevalence of multimorbidity, but could also underlie some of our findings. Participants with one or more missing frailty characteristics were included in the present study, which could resulted in an overrepresentation of 'healthy' participants. This 'survivor effect' may have lead to an underestimations of the associations obtained in this study. In addition, because of the small sample size of frail subpopulations, the current study could have lacked power to, for example, detect an increased risk of mortality and nursing home admission for 'only frail' participants. However, since the confidence intervals of all survival analysis a rather small, it is not likely that a lack of power comprised our results.

In summary, the present study indicates that heterogeneity within the frail populations should not be ignored. Heterogeneity in the frail older adult population present a challenge to understanding whether frailty is a reflection of disease burden or disability or is a true underlying state of vulnerability. The current study identified a subpopulation of older adults ('only frail') who were not at an increased risk for mortality or nursing home admission, but that did display decreased neurocognitive performance and increased CRP levels. Although this subpopulation of frailty appears small, this group will be of use in order to further investigate the pathogenesis of frailty and frail subpopulations.

\section{Funding}

This work was supported by the National Institutes of Health (N01-AG-12100), the National Institute of Aging Intramural Research Program, Hjartavernd (the Icelandic Heart Association), and the Althingi (the Icelandic Parliament). 


\section{REFERENCES}

1. G. Abellan van Kan, Y. Rolland, H. Bergman, J.E. Morley, S.B. Kritchevsky, and B. Vellas. The I.A.N.A Task Force on frailty assessment of older people in clinical practice. J. Nutr. Health Aging, 2008;12(1):29-37.

2. L.P. Fried, C.M. Tangen, J. Walston, A.B. Newman, C. Hirsch, J. Gottdiener, T. Seeman, R. Tracy, W.J. Kop, G. Burke, and M.A. McBurnie. Frailty in older adults: evidence for a phenotype. J. Gerontol. A Biol. Sci. Med. Sci., 2001;56(3):146-156.

3. M.A. Makary, D.L. Segev, P.J. Pronovost, D. Syin, K. Bandeen-Roche, P. Patel, R. Takenaga, L. Devgan, C.G. Holzmueller, J. Tian, and L.P. Fried. Frailty as a predictor of surgical outcomes in older patients. Journal of the American College of Surgeons, 2010;210(6):901-908.

4. C.M. Boyd, Q.L. Xue, C.F. Simpson, J.M. Guralnik, and L.P. Fried. Frailty, hospitalization, and progression of disability in a cohort of disabled older women. Am. J. Med., 2005;118(11):1225-1231.

5. R. Boxer, A. Kleppinger, A. Ahmad, K. Annis, D. Hager, and A. Kenny. The 6-minute walk is associated with frailty and predicts mortality in older adults with heart failure. Congestive heart failure. Greenwich, Conn, 16(5):208-213.

6. L.P. Fried, L. Ferrucci, J. Darer, J.D. Williamson, and G. Anderson. Untangling the concepts of disability, frailty, and comorbidity: implications for improved targeting and care. J. Gerontol. A Biol. Sci. Med. Sci., 2004;59(3):255-263.

7. H.E. Whitson, J.L. Purser, and H.J. Cohen. Frailty thy name is . . Phrailty? J. Gerontol. A. Biol. Sci. Med. Sci., 2007;62(7):728-730.

8. A.B. Newman, J.S. Gottdiener, M.A. McBurnie, C.H. Hirsch, W.J. Kop, R. Tracy, J.D. Walston, and L.P. Fried. Associations of subclinical cardiovascular disease with frailty. J. Gerontol. A. Biol. Sci. Med. Sci., 2001;56(3):158-166.

9. O. Bjornsson, D. Davidsson, H. Olafsson, O. Olafsson, N. Sigfusson, and T. Thorsteinsson. Report ABC XVIII Health Survey in the Reykjavik Area - Men Stages I-III 1967-1969 1970-1971 and 19741976 Participants Invitation Response etc. . 1979.

10. T. Hardarson, M. Gardarsdottir, K.T. Gudmundsson, G. Thorgeirsson, H. Sigvaldason, and N. Sigfusson. The relationship between educational level and mortality. The Reykjavik Study. J. Intern. Med., 2001;249(6):495-502.

11. T.B. Harris, L.J. Launer, G. Eiriksdottir, O. Kjartansson, P.V. Jonsson, G. Sigurdsson, G. Thorgeirsson, T. Aspelund, M.E. Garcia, M.F. Cotch, H.J. Hoffman, and V. Gudnason. Age, Gene/Environment Susceptibility-Reykjavik Study: multidisciplinary applied phenomics. Am. J. Epidemiol., 2007;165(9):1076-1087.

12. Y.Y. Ho, A.M. Matteini, B. Beamer, L. Fried, Q.L. Xue, D.E. Arking, A. Chakravarti, M.D. Fallin, and J. Walston. Exploring biologically relevant pathways in frailty. J. Gerontol. A. Biol. Sci. Med. Sci., 66(9):975-979.

13. S. Katz, A.B. Ford, R.W. Moskowitz, B.A. Jackson, and M.W. Jaffe. Studies of Illness in the Aged. The Index of Adl: A Standardized Measure of Biological and Psychosocial Function. JAMA, 1963;185:914919.

14. M. van den Akker, F. Buntinx, and J. Knottnerus. Comorbidity or multimorbidity: What's in a name. A review of literature. Eur. J. Gen. Pract., 1996;2:65-70.

15. J.M. Guralnik. Assessing the impact of comorbidity in the older population. Annual Epidemiology, 1996;6(5):376-380.

16. A.S. Levey, J.P. Bosch, J.B. Lewis, T. Greene, N. Rogers, and D. Roth. A more accurate method to estimate glomerular filtration rate from serum creatinine: a new prediction equation. Modification of Diet in Renal Disease Study Group. Ann. Intern. Med., 1999;130(6):461-470.

17. E. Sigurdsson, G. Thorgeirsson, H. Sigvaldason, and N. Sigfusson. Unrecognized myocardial infarction: epidemiology, clinical characteristics, and the prognostic role of angina pectoris. The Reykjavik Study. Ann. Intern. Med., 1995;122(2):96-102. 
18. M.F. Folstein, S.E. Folstein, and P.R. McHugh. "Mini-mental state". A practical method for grading the cognitive state of patients for the clinician. J. Psychiatr. Res., 1975;12(3):189-198.

19. M.D. Lezak, D.B. Howieson, and D.W. Loring. Neuropsychological assessment. 2004, New York: OXFORD university press.

20. J.S. Vidal, S. Sigurdsson, M.K. Jonsdottir, G. Eiriksdottir, G. Thorgeirsson, O. Kjartansson, M.E. Garcia, M.A. van Buchem, T.B. Harris, V. Gudnason, and L.J. Launer. Coronary artery calcium, brain function and structure: the AGES-Reykjavik Study. Stroke, 2010;41(5):891-897.

21. A.S. Agatston, W.R. Janowitz, F.J. Hildner, N.R. Zusmer, M. Viamonte, Jr., and R. Detrano. Quantification of coronary artery calcium using ultrafast computed tomography. J. Am. Coll. Cardiol., 1990;15(4):827-832.

22. K.V. Patel, R.D. Semba, L. Ferrucci, A.B. Newman, L.P. Fried, R.B. Wallace, S. Bandinelli, C.S. Phillips, B. Yu, S. Connelly, M.G. Shlipak, P.H. Chaves, L.J. Launer, W.B. Ershler, T.B. Harris, D.L. Longo, and J.M. Guralnik. Red cell distribution width and mortality in older adults: a meta-analysis. J. Gerontol. A Biol. Sci. Med. Sci,. 2010;65(3):258-265.

23. L.S. Gudmundsson, T. Aspelund, A.I. Scher, G. Thorgeirsson, M. Johannsson, L.J. Launer, and V. Gudnason. C-reactive protein in migraine sufferers similar to that of non-migraineurs: the Reykjavik Study. Cephalalgia, 2009;29(12):1301-1310.

24. G. Sigurdsson, T. Aspelund, M. Chang, B. Jonsdottir, S. Sigurdsson, G. Eiriksdottir, A. Gudmundsson, T.B. Harris, V. Gudnason, and T.F. Lang. Increasing sex difference in bone strength in old age: The Age, Gene/Environment Susceptibility-Reykjavik study (AGES-REYKJAVIK). Bone, 2006;39(3):644-651.

25. J.S. Saczynski, M.K. Jonsdottir, M.E. Garcia, P.V. Jonsson, R. Peila, G. Eiriksdottir, E. Olafsdottir, T.B. Harris, V. Gudnason, and L.J. Launer. Cognitive impairment: an increasingly important complication of type 2 diabetes: the age, gene/environment susceptibility--Reykjavik study. Am. J. Epidemiol., 2008;168(10):1132-1139.

26. J.N. Morris, C. Hawes, B.E. Fries, C.D. Phillips, V. Mor, S. Katz, K. Murphy, M.L. Drugovich, and A.S. Friedlob. Designing the national resident assessment instrument for nursing homes. Gerontologist, 1990;30(3):293-307.

27. A.S. Buchman, P.A. Boyle, R.S. Wilson, Y. Tang, and D.A. Bennett. Frailty is associated with incident Alzheimer's disease and cognitive decline in the elderly. Psychosom. Med., 2007;69(5):483-489.

28. L.P. Fried, Q.L. Xue, A.R. Cappola, L. Ferrucci, P. Chaves, R. Varadhan, J.M. Guralnik, S.X. Leng, R.D. Semba, J.D. Walston, C.S. Blaum, and K. Bandeen-Roche. Nonlinear multisystem physiological dysregulation associated with frailty in older women: implications for etiology and treatment. J. Gerontol. A Biol. Sci. Med. Sci., 2009;64(10):1049-1057.

29. R.R. Kalyani, R. Varadhan, C.O. Weiss, L.P. Fried, and A.R. Cappola. Frailty Status and Altered Glucose-Insulin Dynamics. J. Gerontol. A. Biol. Sci. Med. Sci. [Epub ahead of print].

30. S. Goebeler, M. Jylha, and A. Hervonen. Self-reported medical history and self-rated health at age 90. Agreement with medical records. Aging Clin. Exp. Res., 2007;19(3):213-219. 


\section{APPENDIX}

Prevalence (and percentage) of medical conditions included in the present study

\begin{tabular}{lccccc}
\hline & $\begin{array}{c}\text { Nonfrail } \\
(\mathrm{n}=3941)\end{array}$ & $\begin{array}{c}\text { Only Frail } \\
(\mathrm{n}=64)\end{array}$ & $\begin{array}{c}\text { FD } \\
(\mathrm{n}=94)\end{array}$ & $\begin{array}{c}\text { FM } \\
(\mathrm{n}=125)\end{array}$ & $\begin{array}{c}\text { FDM } \\
(\mathrm{n}=190)\end{array}$ \\
\hline Diabetes & $451(11.4)$ & $4(6.3)$ & $6(6.4)$ & $18(14.4)$ & $44(23.2)$ \\
Dementia & $150(3.8)$ & $3(4.7)$ & $9(9.6)$ & $20(16.0)$ & $38(20.0)$ \\
Anemia & $280(7.1)$ & $3(4.7)$ & $2(2.1)$ & $35(28.0)$ & $46(24.2)$ \\
Osteoporosis & $78(2.0)$ & 0 & $1(1.1)$ & $3(2.4)$ & $11(5.8)$ \\
Arthritis & $1440(36.5)$ & $13(20.3)$ & $27(28.7)$ & $68(54.4)$ & $106(55.8)$ \\
Cancer & $493(12.5)$ & $4(6.3)$ & $7(7.4)$ & $31(24.8)$ & $51(26.8)$ \\
Coronary Heart Disease & $805(20.4)$ & $5(7.8)$ & $6(6.4)$ & $41(32.8)$ & $68(35.8)$ \\
Kidney disease & $1142(29.0)$ & $7(10.9)$ & $3(3.2)$ & $76(60.8)$ & $101(53.2)$ \\
Liver disease & $54(1.4)$ & $1(1.6)$ & $2(2.1)$ & $3(2.4)$ & 0 \\
Fractures (pelvis/hip) & $86(2.2)$ & $1(1.6)$ & 0 & $6(4.8)$ & $14(7.4)$ \\
Asthma & $532(3.1)$ & $3(4.7)$ & $2(2.1)$ & 24 & $45(23.7)$ \\
COPD & $123(3.1)$ & 0 & $2(2.1)$ & $6(4.8)$ & $16(8.4)$ \\
Stroke & $197(5.0)$ & $3(4.7)$ & $2(2.1)$ & $17(13.6)$ & $34(17.9)$ \\
\hline
\end{tabular}




\section{CHAPTER 6}

\section{Diabetes mellitus type II as a risk factor for depression: a lower than expected risk in a general practice setting}

Published as:

S. Aarts, M. van den Akker, M.P. van Boxtel, J. Jolles, B. Winkens, and J.F. Metsemakers. Diabetes mellitus type II as a risk factor for depression: a lower than expected risk in a general practice setting. European Journal of Epidemiology 2009:24(10):641-648. 


\section{ABSTRACT}

\section{Background}

The aim of the present study was to determine whether a diagnosis of diabetes mellitus $(\mathrm{DM})$ in a primary setting is associated with an increased risk of subsequent depression.

\section{Methods}

A retrospective cohort design was used based on the Registration Network Family Practice (RNH) database. Patients diagnosed with diabetes mellitus at or after the age of 40 and who were diagnosed between 01-01-1980 and 01-01-2007 $(N=6140)$, were compared with age-matched controls from a reference group $(N=18416)$ without a history of diabetes. Both groups were followed for an emerging first diagnosis of depression (and/or depressive feelings) until January 1, 2008.

\section{Results}

$2.0 \%$ of the people diagnosed with diabetes mellitus developed a depressive disorder, compared to $1.6 \%$ of the reference group. After statistical correction for confounding factors diabetes mellitus was associated with an increased risk of developing subsequent depression (HR 1.26; 95\% CI: 1.12-1.42) and/or depressive feelings (HR 1.33; 95\% CI: 1.18-1.46). After statistical adjustment practice identification code, age and depression preceding diabetes, were significantly related to a diagnosis of depression.

\section{Conclusions}

Patients with diabetes mellitus are more likely to develop subsequent depression than persons without a history of diabetes. Results from this large longitudinal study based on a general practice population indicate that this association is weaker than previously found in cross-sectional research using self-report surveys. Several explanations for this dissimilarity are discussed. 


\section{INTRODUCTION}

It is generally known that co-morbid depression is highly prevalent in persons diagnosed with a chronic illness ${ }^{1}$. Especially, the co-occurrence of depression in persons diagnosed with diabetes mellitus has been a major topic in recent epidemiologic research. This research suggests that depression is more prevalent among adults with diabetes than in those without this condition ${ }^{2-4}$. More precisely, a review and meta-analysis involving 42 cross-sectional studies by Anderson et al. (2004) suggests that diabetes doubles the odds of a co-morbid depression ${ }^{5}$.

The impact of depression on patients with diabetes has also been an area of interest. Gonzales and colleagues (2007) defined co-morbid depression as a risk factor for nonadherence to important aspects of diabetes self-care, such as medication and diet regimens ${ }^{6}$. In addition, diabetic patients with a depression reported a greater number of physical symptoms related to diabetes ${ }^{4}$, and showed lower levels of metabolic control ${ }^{7}$ and quality of life ${ }^{8}$.

In the past decade, literature on the association between diabetes mellitus and depression has grown considerably. However, the temporal or causal relationship between diabetes mellitus and depression still remains unclear since only few longitudinal studies have evaluated diabetes as a risk factor for depression?. Most studies that were of longitudinal nature focused on the relation between depression and the development of a subsequent diagnosis of diabetes mellitus ${ }^{10-12}$ have produced contradictory results ${ }^{10,13,14}$. The few studies that did focus on the longitudinal relation between diabetes and subsequent depressive disorder showed that diabetes type II is associated with an increased risk of developing depressive symptoms $s^{13,15,16}$. However, these studies were either restricted to small populations ${ }^{16}$ or lacked a proper adjustment for the presence of other chronic

diseases 13, 15. Consequently, the results of these studies must be considered less representative for the general population.

Finally, another major drawback in previous mentioned longitudinal studies concerns the use of self-report surveys to identify depression (e.g. CES-D ${ }^{11,13}{ }^{16}, \mathrm{HADS}^{6}$, BDI-II ${ }^{17}$, $\left.\mathrm{PHQ}^{4}\right)$ or self-reported diabetes ${ }^{16}$. An important consequence of this differential assessment is the wide variability in the presence and strength of the established association between diabetes and depression ${ }^{11}$. Besides, self-report surveys may not be the most appropriate measure for the assessment of depressive disorder for several reasons. Data from self-report scales cannot be used for making a clinical diagnosis of depression. Moreover, by using different cut-off points to define depression ${ }^{5}$, comparison between these studies is hindered. Finally, health questionnaires are known to suffer from several conceptual problems. For example, the results of self-reports and questionnaires are based on a particular point in time that frequently do not include information about the intensity or consistency of the complaints or problems, personal growth or coping strategies ${ }^{18}$.

In the present study, the previously mentioned drawbacks were defied. The aim of the present study was to assess the risk of depression over time in patients with diabetes mellitus compared with those without a history of diabetes. For this purpose, a large retrospective cohort study was performed in a large general practice setting. 


\section{METHODS}

\section{Sample Frame}

The present study was carried out within the context of the Registration Network Family Practices (RegistratieNet Huisartspraktijken, RNH). Descriptive background characteristics of samples in other studies which made use of the RNH database were found to be comparable to the Dutch population ${ }^{19}$. The $\mathrm{RNH}$ is a continuously updated database, which contains the medical records of patients from 21 family practices in which 65 general practitioners (GPs) working in the south of the Netherlands are participating. This database includes all relevant current and past health problems. A health problem is defined as 'anything that has required, does or may require health care management and has affected or could significantly affect a person's physical or emotional well-being' ${ }^{20}$. These problems are coded in a standardized fashion, according to the International Classification of Primary Care (ICPC), using the criteria of the International Classification of health in Primary Care (ICHPPC-2) ${ }^{21}$ and other more current guidelines of the Dutch College of GP's. A diagnosis is made by the GP or by a medical specialist who is consulted by the GP. Especially in complex medical conditions, registration is often based on a specialist diagnosis reported to the GP. In general, health problems are only coded by the GP when they are permanent (no recovery expected), chronic (duration longer than 6 months) or recurrent (more than three recurrences within 6 months), or when they have lasting consequences for the functional status or prognosis of the patient. The database also contains background information on the patient's sex, date of birth, marital status, type of household, practice identification code and level of education. Membership of the RNH population ends by migration or death. All patients included in the RNH database have been informed about the anonymous use of their health information and are removed from the database if desired. The quality of the data is ascertained by ample instruction and training sessions, regular regional consensus groups, quality control audits, an online thesaurus available during data-entry and systematic control for erroneous or missing entries $^{20}$.

\section{Diagnostic Criteria}

ICPC is now widely used in Europe as a diagnostic classification system, which has relations both with ICD-9 and with other ICD-9 derived systems being used in primary care $^{21}$. Modified international criteria are followed, as expressed in the guidelines of the Dutch College of General Practitioners, for the diagnosis of Diabetes Mellitus (ICPC code T90) $)^{21}$

The diagnosis of DM requires an elevated glucose level [fasting plasma glucose levels of $124 \mathrm{mg} / \mathrm{dl}(6.9 \mathrm{mmol} / \mathrm{l})$ or more, a fasting capillary glucose level of $108 \mathrm{mg} / \mathrm{dl}(6.0$ $\mathrm{mmol} / \mathrm{l})$ or more, or non-fasting plasma or capillary glucose level of $198 \mathrm{mg} / \mathrm{dl}(11.0$ $\mathrm{mmol} / \mathrm{l})$ ] that is confirmed using a fasting glucose level a few days later. Since the disease contents and progress between type I and type II diabetes may vary considerably, the present study was aimed at patients with type II diabetes. In order to diminish the number 
of patients with DM type I in the present study, only patients diagnosed with DM at or after the age of 40 years were included.

The diagnosis of depressive disorder (ICPC code P76) was made in a diagnostic interview conducted by either a general practitioner or a specialist. According to ICPC criteria, patients should not be psychotic and comply with at least three of the following six criteria: (i) sadness or melancholy more than can be explained by the psychosocial stress, (ii) suicidal thoughts or attempt, (iii) indecisiveness, decreased interest in usual activities or diminished ability to think, (iv) feelings of worthlessness, self-reproach, or inappropriate or excessive guilt, (v) early morning wakening, hypersomnia, or early morning fatigue, or (vi) anxiety, hyperirritability, or agitation. The nine symptoms of a depressive episode described in the DSM-IV of the American Psychiatric Association are equivalent to the six criteria of the RNH following ICPC code $\mathrm{P}^{21} \mathrm{6}^{2}$. Patients who presented themselves with chronic or recurrent depressive feelings, but who did not fully complied with the requirements for depressive disorder (ICPC code P76) were coded as having depressive feelings (ICPC code P03).

\section{Study Design}

Data were drawn from the RNH database available on January 1, 2008. Patients with DM in our study were diagnosed with ICPC code T90 (Diabetes Mellitus Type I and Type II) between January 1, 1980 and January 1, 2007. In order to restrict the number of patients with type I diabetes, only patients with diabetes mellitus who were 40 years or older at the time of diagnosis were included $(\mathrm{N}=6140)^{3,22}$.

The reference group consisted of subjects with no history of DM. In order to increase the power of the current study, individuals in the reference group were matched to the diabetes patients by age (year of birth) and were assigned the date of diagnosis of their matched counterparts as the starting date for their follow-up period. Each patient was matched (according to year of birth) to 3 controls in the reference group ( $N=18416$ ), except for 4 patients, who could only be matched to 2 controls from the reference group. Each subject was followed for an emerging first diagnosis of a depressive disorder. Followup ended on January 1, 2008, or earlier in case of a diagnosis of depressive disorder or due to censoring (i.e. migration or death). In the sensitivity analyses, each subject was followed for an emerging first diagnosis of a depressive disorder or the emergence of depressive feelings (ICPC code P03). Follow-up ended on January 1, 2008, or earlier in case of a diagnosis of depressive disorder, depressive feelings or due to censoring (i.e. migration or death).

\section{Statistical Analysis}

Statistical analyses were conducted using the SPSS statistical software package version 16.0 for Windows (SPSS Inc., Chicago, IL, USA). An independent T-test was used to examine a possible difference in mean follow-up time between the diabetes and the reference group. Cox proportional hazards survival analysis was applied in which the effect of diabetes on depression was corrected for age (ranging from 40 to 97 year), gender, level of education (3 levels: low, intermediate, and high), number of chronic co-morbid diseases in 7 categories 
(ranging from 0 to 6 diseases, in which the last category coded for 6 or more diseases) (see Appendix A.), the practice where a patient was registered (practice identification code) and a diagnosis of depression preceding the start of follow-up. The latter variable coded for all subjects who had a diagnosis of depression (and/or depressive feelings in case of the sensitivity analysis) before the starting date of the follow-up period.

All potential confounders in the analyses were declared as categorical and coded into dummy variables. Since patients were matched with controls by age (in years), age was also declared as a categorical variable. Hazards ratios (HR) and 95\% confidence intervals (CI) are reported. For the potential confounders the p-value of the survival analysis was reported. Potential confounders who were not significantly related ( $\mathrm{P}$-values $>0.05$ ) to a subsequent diagnosis of depression were excluded from the final analyses using a backward method. Two kinds of analyses were conducted. The first analysis made use of the diagnosis of depression as event outcome. The second analysis was a sensitivity analysis, in which the diagnosis of depressive feelings, not complying with the requirements for depressive disorder, was pooled with the diagnosis of depression as event outcome.

\section{RESULTS}

Descriptive characteristics of the study sample are reported in Table 1. A significant difference in time until diagnosis of depressive disorder between the two groups was found $(\mathrm{P}<0.001)$.

The mean follow-up was 7.7 years $(\mathrm{SD}=5.7)$ for the diabetes group and 7.9 years $(\mathrm{SD}=$ 6.6) for the reference group. During the follow-up period, 122 patients $(2.0 \%)$ with diabetes mellitus and 295 persons $(1.6 \%)$ of the reference group developed a depressive disorder.

Gender, co-morbidity and level of education did not have a significant effect and were therefore removed as covariates from all statistical analyses. A diagnosis of diabetes mellitus was significantly associated with a greater likelihood of developing subsequent diagnosis of depression (HR 1.32; 95\% CI: 1.19-1.48). Statistical adjustment for the remaining confounders (age, practice identification code and depression preceding DM) attenuated the strength of this association, but it remained statistical significant (HR 1.26; 95\% CI: 1.12-1.42) (see Figure 1). Adding depressive feelings to the event outcome yielded similar results. During follow up diabetes patients were significantly more likely to develop a depressive disorder and/or depressive feelings (HR 1.27; 95\% CI: 1.15-1.41). After controlling for confounding factors (age, practice identification code and depression preceding DM) this association became even slightly stronger (HR 1.33; 95\% CI: 1.18 1.46) (see Figure 2). After adjusting for the other variables, practice identification ( $\mathrm{P}=$ $0.004)$, age $(\mathrm{P}<0.001)$ and a diagnosis of depression preceding $\mathrm{DM}(\mathrm{P}<0.001)$ code also showed a significant association with risk of depression. 
Table 1. Descriptive characteristics of study population

\begin{tabular}{lcc}
\hline Characteristics & $\begin{array}{c}\text { Patients } \\
(\mathrm{n}=6140)\end{array}$ & $\begin{array}{c}\text { Controls } \\
(\mathrm{n}=18416)\end{array}$ \\
\hline No. of subjects with depression preceding diabetes (\%) & $325(5.3)$ & $863(4.7)$ \\
No. of subjects with depression after diabetes (\%) & $122(2.0)$ & $295(1.6)$ \\
Mean age in years (SD)a & $63.8(11.2)$ & $63.8(11.2)$ \\
Mean number of follow-up years (Range) & $7.7(0-28)$ & $7.9(0-28)$ \\
Gender & & \\
Males (\%) & $2953(48.1)$ & $8519(46.3)$ \\
Females (\%) & $3187(51.9)$ & $9897(53.7)$ \\
Number of co-morbid diseases (\%) & \\
0 & $0(0.0)$ & $3325(18.1)$ \\
1 & $0(0.0)$ & $3844(20.9)$ \\
2 & $566(9.2)$ & $3367(18.3)$ \\
3 & $1003(16.3)$ & $2580(14.0)$ \\
4 & $1040(16.9)$ & $1950(10.6)$ \\
5 & $929(15.1)$ & $1377(7.5)$ \\
$\geq 6$ & $2602(42.4)$ & $1973(10.7)$ \\
Educational Level & & $9483(51.5)$ \\
Low (\%) & & $4143(81.4)$ \\
Medium (\%) $\%)$ & $3052(49.7)$ & $1460(7.9)$ \\
\hline
\end{tabular}

Mean age described as date of inclusion cohort For $30.6 \%$ of the diabetic group and $18.1 \%$ of the reference group educational information was missing or not updated; low is defined as primary school and/or lower vocational education, medium as secondary school and/or medium level vocational education, high as higher vocational education and/or university.

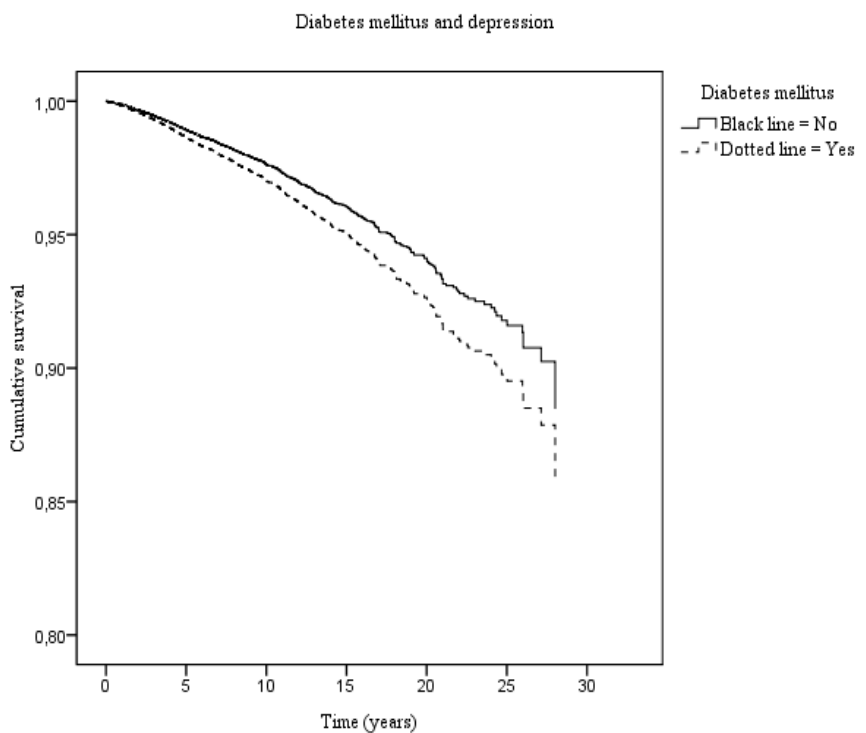

Figure 1. Survival curve stratified for age, practice identification code and a diagnosis of depression preceding DM. 


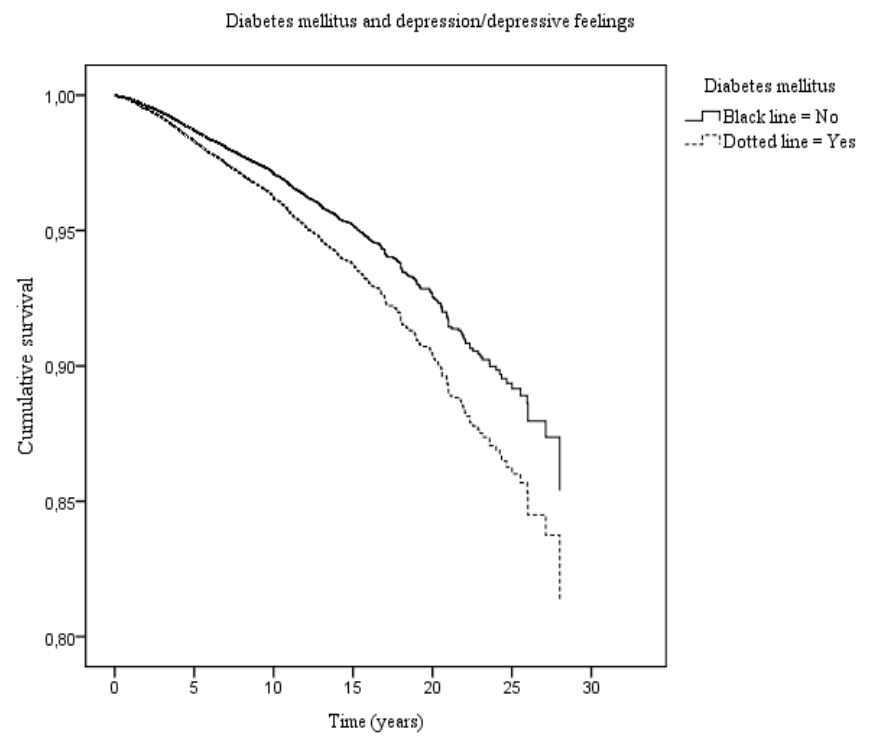

Figure 2. Survival curve stratified for age, practice identification code and a diagnosis of depression preceding DM.

\section{DISCUSSION}

The present study revealed that a diagnosis of diabetes mellitus type II at or after the age of forty was associated with an increased likelihood of developing a subsequent depression or depressive feelings. After statistical adjustment, practice identification code, age and a diagnosis of depression preceding the diagnosis of diabetes mellitus appeared to be significantly related to a subsequent diagnosis of depression.

This is the first longitudinal study on the association between diabetes mellitus and subsequent depression based on data from general practices. A meta-analysis by Anderson et al. (2001) involving 42 cross-sectional studies reported that patients with type II diabetes are twice more likely to experience depressive symptoms than their peers without diabetes 5 . In contrast, the present results suggest that patients with diabetes mellitus are 1.12-1.41 (CI) times more likely to develop depression and 1.18-1.46 times (CI) more likely to develop depression and/or depressive feelings. Our findings confirm the outcome of earlier studies in showing an increased incidence of depression among patients diagnosed with diabetes mellitus. However, this association appears to be weaker than found in earlier studies. This discrepancy can be due to a number of reasons. Firstly, it has been shown that depression rates are two to three times higher in studies that use self-reports ${ }^{5}$. For example, self-report measures may identify a broader spectrum of depressive disorders or symptoms that may reflect co-morbid psychiatric illness or general distress ${ }^{5}$, which could result in an overestimation of the prevalence of depression ${ }^{13}$. Secondly, using a diagnosis of depression preceding DM as a confounder can have reduced the association between diabetes type II 
and depression. Although the association between diabetes and depression attenuated without this correction, it remained statistical significant. Finally, in the present study GPs were not instructed to systematically screen patients for possible depression or depressive symptoms which could have led to a lower risk estimate. If this is indeed the case, the present study reports an underestimation of depression in diabetes patients. It is important for GPs to be aware of the fact that patients with type II diabetes are more likely to experience depression than their peers without diabetes. Several studies have shown that adherence to a variety of self-care activities ${ }^{6}$ and metabolic control ${ }^{7}$ decreases in diabetes patient as a consequence of co-morbid depression. Diabetes patients with co-morbid depression also show a decline in quality of life ${ }^{8}$. Moreover, for approximately $75 \%$ of the people diagnosed with diabetes mellitus in the Netherlands, the GP is the primary medical caregiver ${ }^{23}$, making the GP the proficient person to detect depression or depressive feelings. The present findings imply that GPs who are consulted by patients diagnosed with DM type II should be especially aware of the patient's increased risk of developing depression in the near future. In short, general practitioners should be alert to possible early signs of depression in diabetic patients to ensure early detection and possibly even prevention of a depressive disorder. Nurse practitioners for diabetes mellitus, who assist the general practitioners and provide a broad range of health care services, could fulfil an important role in this aspect. Nurse practitioners focus on patients' conditions as well as on the effects of the illness on the lives of the patients and their families and can therefore serve as a "point of entry" for physical as well as mental problems diabetes patients encounter. Nurse practitioners could enhance diagnostics by systematically screen diabetes patients for possible depression or depressive symptoms in order to prevent underdiagnosis of depression in diabetes patients.

Another intriguing finding of the present study was that practice identification code was significantly associated with an enhanced likelihood of developing a depression. It appears that in 3 of the 21 practices involved, a relatively high percentage of patients is diagnosed with depression (ranging from 9.5 to $9.8 \%$ of the patients) while in 3 of the 21 practices this percentage is noticeably smaller (ranging from 4.0 to $4.7 \%$ of the patients). Hence, it seems plausible that GPs may differ regarding their inclination to diagnose a depression. After investigating the most important characteristics of the practices incorporated in the present study, such as geographic place (defined by postal code) of a general practice, total number of diagnosed depressive disorders in the general practice, number and gender of patients, education of the patients, and number and gender of GPs in a practice, we were not able to identify any specific characteristics that could explain this effect. This diagnostic variability may have important implications for general practices ${ }^{24}$.

Our study has several advantages over previous studies. This is the first longitudinal study that evaluated the causal relationship between diabetes mellitus type II and subsequent occurrence of depressive disorder and/or depressive feelings in a general practice based setting. Consequently, the results seem more representative for the general population than results of studies conducted in smaller and more homogeneous samples ${ }^{17,25}$. Also, the sample size used in the present study supports the robustness of our risk estimates. Moreover, prior studies commonly relied on a variety of self-report surveys 
which are known to overestimate the prevalence of depression ${ }^{5}$. The six criteria following diagnosis of depressive disorder in the RNH database are essentially comparable to the nine symptoms of a depressive episode described in the DSM-IV of the American Psychiatric Association $^{21}$. These criteria are a solid foundation for making a uniform diagnosis of depression by GPs.

Despite the previous mentioned strengths, our findings must be interpreted in light of some possible limitations. First, since the RNH database does not make a clear distinction between diabetes type I and type II, the present study only included patients diagnosed with diabetes mellitus at or after the age of 40 years. Consequently, it cannot be ruled out that none of the diabetes type I patients were included. Second, the total number of conditions registered in the RNH database reflects the GPs perspective of the health status and relevant health problems of his patients. As a result, some health problems may be missing because the patient did not report them to the GP or because the GP does not judge them to be clinically significant ${ }^{26}$. The number of missing health problems, however, appears to be rather small ${ }^{27}$. Furthermore, GPs have a tendency to use a diagnosis primarily as a mean to reach the goal of helping the patient and not as a goal in itself ${ }^{18}$. This is not the case in questionnaires and self-reports, which could have resulted in an underestimation of the prevalence of depression. Due to the very large follow-up period (01-01-1980 to 01-01-2008) it may be argued that no state of the art impression of the association between diabetes and subsequent depression is given. However, an additional analysis involving a smaller, more recent period of time (01-01-1995 to 01-01-2008) yielded similar results. Moreover, an analysis with a follow-up period of only 6 months was also conducted, which gave similar results. Finally, one of the drawbacks of studies that combine data from multiple practices is the between practice variability ${ }^{27}$ : it is generally assumed that some variability exists between general practitioners in making a diagnose ${ }^{24}$. Possibly, this between practice variability may have resulted in the effect that code of practice has on the development of depression.

Further research is warranted to investigate a multitude of unanswered questions. More well-conducted research with adequate control for confounding factors is needed to investigate the causal relationship between diabetes and depression mellitus more in depth. In particular, the influence of practice setting on the longitudinal association between diabetes and depression should be studied in more detail. Future research should also explore the role of other confounding factors. For example, the influence of psychosocial and social-economic factors on the association between diabetes and depression needs to be elucidated.

In conclusion, the present research adds to the evidence concerning the association between diabetes mellitus type II and depression, in that this association also holds in a longitudinal setting in a large general practice population. The present results indicate, however, that patients with diabetes mellitus are less likely to develop a subsequent depression than was expected based on previous research. 


\section{REFERENCES}

1. B.W. Penninx, A.T. Beekman, J. Ormel, D.M. Kriegsman, A.J. Boeke, J.T. van Eijk, and D.J. Deeg. Psychological status among elderly people with chronic diseases: does type of disease play a part? J. Psychosom. Res., 1996;40(5):521-534.

2. K.D. Barnard, T.C. Skinner, and R. Peveler. The prevalence of co-morbid depression in adults with Type 1 diabetes: systematic literature review. Diabetes Medicine. 2006;23(4):445-448.

3. C. Li, E.S. Ford, T.W. Strine, and A.H. Mokdad. Prevalence of depression among U.S. adults with diabetes: findings from the 2006 behavioral risk factor surveillance system. Diabetes Care, 2008;31(1):105-107.

4. E.J. Ludman, W. Katon, J. Russo, M. Von Korff, G. Simon, P. Ciechanowski, E. Lin, T. Bush, E. Walker, and B. Young. Depression and diabetes symptom burden. Gen. Hosp. Psychiatry, 2004;26(6):430-436.

5. R.J. Anderson, R.E. Clouse, K.E. Freedland, and P.J. Lustman. The prevalence of comorbid depression in adults with diabetes. A meta-analysis. Diabetes Care, 2001;24(6):1069-1078.

6. J.S. Gonzalez, S.A. Safren, E. Cagliero, D.J. Wexler, L. Delahanty, E. Wittenberg, M.A. Blais, J.B. Meigs, and R.W. Grant. Depression, self-care, and medication adherence in type 2 diabetes: relationships across the full range of symptom severity. Diabetes Care, 2007;30(9):2222-2227.

7. P.J. Lustman and R.E. Clouse. Depression in diabetic patients: the relationship between mood and glycemic control. J. Diabet. Complications, 2005;19(2):113-122.

8. G.A. Brenes. Anxiety, depression, and quality of life in primary care patients. J. Clin. Psychiatry, 2007;9(6):437-443.

9. L.V. Kessing, F.M. Nilsson, V. Siersma, and P.K. Andersen. No increased risk of developing depression in diabetes compared to other chronic illness. Diabetes Res. Clin. Pract., 2003;62(2):113-121.

10. M. van den Akker, A.G. Schuurman, J.F.M. Metsemakers, and F. Buntinx. Is depression related to subsequent diabetes mellitus? Acta Psychiatr. Scand., 2004;110:178-183.

11. M.R. Carnethon, L.S. Kinder, J.M. Fair, R.S. Stafford, and S.P. Fortman. Symptoms of depression as a risk factor for incident diabetes: findings from the national health and nutrition examination epidemiologic follow-up study, 1971-1992. Am. J. Epidemiol., 2003;158:416-423.

12. M.J. Knol, J.W. Twisk, A.T. Beekman, R.J. Heine, F.J. Snoek, and F. Pouwer. Depression as a risk factor for the onset of type 2 diabetes mellitus. A meta-analysis. Diabetologia, 2006;49(5):837-845.

13. S.H. Golden, M. Lazo, M. Carnethon, A.G. Bertoni, P.J. Schreiner, A.V. Roux, H.B. Lee, and C. Lyketsos. Examining a bidirectional association between depressive symptoms and diabetes. JAMA, 2008;299(23):2751-2759.

14. L.V. Kessing, F.M. Nilsson, V. Siersma, and P.K. Andersen. Increased risk of developing diabetes in depressive and bipolar disorders? J. Psychiatr. Res., 2004;38(4):395-402.

15. P. de Jonge, J.F. Roy, P. Saz, G. Marcos, and A. Lobo. Prevalent and incident depression in community-dwelling elderly persons with diabetes mellitus: results from the ZARADEMP project. Diabetologia, 2006;49(11):2627-2633.

16. C. Maraldi, S. Volpato, B.W. Penninx, K. Yaffe, E.M. Simonsick, E.S. Strotmeyer, M. Cesari, S.B. Kritchevsky, S. Perry, H.N. Ayonayon, and M. Pahor. Diabetes mellitus, glycemic control, and incident depressive symptoms among 70- to 79-year-old persons: the health, aging, and body composition study. Arch. Intern. Med., 2007;167(11):1137-1144.

17. M. de Groot, T. Doyle, E. Hockman, C. Wheeler, B. Pinkerman, J. Shubrook, R. Gotfried, and F. Schwartz. Depression Among Type 2 Diabetes Rural Appalachian Clinic Attendees. Diabetes Care, 2007;30(6):1602-1604.

18. F. Buntinx, J. De Lepeleire, J. Heyrman, B. Fischler, D. Vander Mijnsbrugge, and M. Van den Akker. Diagnosing depression: what's in a name? Eur. J. Gen. Pract., 2004;10(4):162-165.

19. J.F.M. Metsemakers, J.A. Knottnerus, G.J. van Schendel, R.J. Kocken, and C.B. Limonard. Unlocking patients' records in general practice for research, medical education and quality assurance: the Registration Network Family Practices. Int. J. Biomed. Comput., 1996;42(1-2):43-50. 


\section{Chapter 6}

20. J.F.M. Metsemakers, P. Höppener, J. Knottnerus, and C. Limonard. Computerized health information in the Netherlands: a registration network of family practices. Br. J. Gen. Pract. 1992;42(356):102_ 106.

21. C.c.o. WONCA. ICPC International Classification of Primary Care. 1987, Oxford: Oxford Univesity Press.

22. F. Petrak, J. Hardt, H.U. Wittchen, B. Kulzer, A. Hirsch, F. Hentzelt, K. Borck, F. Jacobi, U.T. Egle, and S.O. Hoffmann. Prevalence of psychiatric disorders in an onset cohort of adults with type 1 diabetes. Diabetes. Metab. Res. Rev., 2003;19(3):216-222.

23. C.A. Baan, H. J.H., and P.M. Rijken, Afstemming in de zorg. Een achtergrondstudie naar de zorg voor mensen met een chronische aandoening. RIVM rapport. 2003, RIVM/NIVEL: Bilthoven.

24. D.L. Crombie, K.W. Cross, and D.M. Fleming. The problem of diagnostic variability in general practice. J. Epidemiol. Community Health, 1992;46(4):447-454.

25. N. Kawakami, N. Takatsuka, H. Shimizu, and H. Ishibashi. Depressive symptoms and occurrence of type 2 diabetes among Japanese men. Diabetes Care, 1999;22(7):1071-1076.

26. M. van den Akker, F. Buntinx, J.F. Metsemakers, S. Roos, and J.A. Knottnerus. Multimorbidity in general practice: prevalence, incidence, and determinants of co-occurring chronic and recurrent diseases. J. Clin. Epidemiol., 1998;51(5):367-375.

27. J.F. Metsemakers, J.A. Knottnerus, G.J. van Schendel, R.J. Kocken, and C.B. Limonard. Unlocking patients' records in general practice for research, medical education and quality assurance: the Registration Network Family Practices. Int. J. Biomed. Comput., 1996;42(1-2):43-50. 


\section{CHAPTER 7}

Exploring medical data to generate new hypotheses: an introduction to data and text mining techniques in epidemiology

Submitted as:

S. Aarts, R. Vos, M.P. van Boxtel, F.R.J. Verhey, J.F. Metsemakers and M. van den Akker. 


\section{ABSTRACT}

\section{Background}

To date, we are confronted with large collections of medical datasets and medical documents. The aim of the present article is to get the medical researcher acquainted with the emerging field of Knowledge Discovery in Databases (KDD), which can aid researchers in the context of hypothesis generation.

\section{Methods}

Descriptive study based on literature. A summary about what KDD is and how it can be used to extract information from medical data and documents is provided. The add-on value of KDD applications over and above traditional statistical methods is outlined. Examples of KDD applications useful in (bio)medical research are provided.

\section{Results}

KDD extends traditional statistical methods by providing useful tools for exploratory research in large amounts of medical data and documents in order to detect (hidden) patterns, trends, and anomalies. Rather than to serve as a golden standard, the application of KDD is aimed at supporting a (bio)medical expert's knowledge and decisions.

\section{Conclusions}

KDD can explore and model large amounts of (bio)medical data and documents in a (semi)automatical matter to extract previously unknown, hidden and potentially useful information. Although KDD is not a universal solution it can provide innovative and useful tools for (bio)medical research. 


\section{INTRODUCTION}

Today, many health care workers systematically collect electronic patient data (Electronic Patient Records, EPRs) as part of their daily routine. When EPRs were first introduced these new databases were considered as an important tool for improving quality of care, since these medical databases encompass an important amount of information, including details about patient encounters, drug prescriptions and disease diagnosis. These relatively new types of comprehensive databases enable medical researchers to provide answers on many health-related research questions. Examples of such questions are 'Does drug A and drug B cause adverse drug reactions when provided together to cure disease X?', 'Is comorbid disease A in people diagnosed with disease B associated with a lower quality of life when compared to people who are only diagnosed with disease B?' or 'Is gene A a predictor for the development of disease X?'. They are derived from a priori postulated hypotheses and are therefore tested relatively simply using standard statistical analyzing methods.

Large medical databases can also provide the opportunity to conduct more exploratory bottom-up research, which exists without a priori postulated hypotheses. This may include research questions such as 'Which combinations of drugs can cause adverse drug reactions?, 'Which disease combinations may lead to a significant decrease in quality of life?' and 'Which genes are predictive for disease X'? In contrast to the first set of research questions which are part of the practice of hypothesis testing, the latter type of questions ideally fit within hypothesis-generating research.

Although standard statistical techniques are indeed adequate and useful to evaluate a priori postulated hypotheses, they are far less useful in the practice of hypothesis-generating research. Moreover, standard statistical techniques are adequate and useful to process moderate amounts of data but are less efficient when the amount and complexity of data increases ${ }^{1}$. When analyzing large and complex data sets with traditional statistical techniques, the basic problem is that the number of predictive variables be limited a priori. As a result, information about relationships that are not obvious or patterns that are not expected, but are present in the discarded variables, will remain undiscovered. As a result of the lack of ability to extract new information and insight from these large amounts of medical data, (bio)medical researchers and epidemiologists may be missing a significant portion of medical relevant information. Hence, there is an immediate call for new techniques which enable researchers to analyze large amounts of data in a fast and (semi)automatically manner without the need of postulating a priori hypotheses.

The need to extract meaningful information and patterns from observed data has always been an integral part of science, especially since pattern detection is essential for the construction and testing of scientific hypotheses and causal models. Since new associations and patterns can be found in large (bio)medical databases which could benefit individual patients as well as society, new analytical techniques have been applied to investigate the hidden scientific treasures contained in medical databases and in the medical literature ${ }^{2}$. For example, Harpez and collegues evaluated 162.844 reports of suspected Adverse Drug 
Events (ADEs) in order to investigate which combinations of two or more drugs may cause ADEs ${ }^{3}$. Using a well established data mining method called association rules they were able to explore adverse drug events without postulating any a priori assumptions. Of the 2.603 produced association rules, 1.167 contained three or more drugs and $67 \%$ of the found associations were already known, suggesting that the data mining method was a valid approach for the identification of ADEs. Some drug combinations that were found, including the combination cyclophosphamide, doxorubicin, prednisone and rituximab, which was reported to cause febrile neutropenia, was not previously identified as an ADE. Another intriguing finding of this study was that atorvastatin in combination with lisinopril was found to cause dyspnoea, which also had not been previously recognized ${ }^{3}$.

Piatetsky-Shapiro was the first to introduce the term Knowledge Discovery in Databases (KDD) when referring to the process of identifying valid, novel, potentially useful, and comprehensible patterns in complex and large data sets, without the postulation of a priori hypotheses ${ }^{4}$. Indeed, the primary aim of knowledge discovery is to infer implicit and hidden patterns into well-grounded and testable hypotheses ${ }^{5}$. In contrast to traditional statistical analyzing methods, KDD allows much more variables (in data) and text (in documents) to be examined in relationship to one another and to the outcome in a more efficient, inexpensive and objective manner ${ }^{6}$. For example, a study by Swindell and colleagues (2010) was able to include as much as 377 different patient characteristics in 4.097 subjects to identify the factors predictive for successful aging in older women ${ }^{7}$. The multivariate model, using a cross-validation approach involving random splitting of subjects into "training" and "testing" sets (explained below) integrated these 377 factors to represent a multidimensional 'healthy aging' phenotype.

In this paper we want to provide an overview of what KDD techniques can offer to biomedicine and epidemiology and how it can be applied in health care research. We will focus on two sources of information; (large) medical databases, in particular those involving patient records, and repositories of (bio)medical literature (e.g. Medline/Pubmed). This paper is not intended to provide an exhaustive overview of all available knowledge discovery techniques. Rather, it is aimed at giving a concise and comprehensible outline of knowledge discovery by providing the mere value of KDD over statistical exploratory research and to discuss several interesting applications of KDD that are useful in the practice of hypothesis generating in epidemiology and (bio)medical research.

\section{TRADITIONAL STATISTICAL TECHNIQUES VERSUS KDD}

In medical research two general approaches can be discerned: a closed process, which is defined by testing hypotheses, and an open discovery process, which is characterised by the generation of hypotheses 5 . Traditional statistical techniques are defined as serving a closed approach; the researcher should know in advance what he is looking for, based on previously conducted research and his own knowledge and experience. In pursuing this route, the researcher will not easily find something outside his direct scope of interest ${ }^{8}$. Moreover, researchers are subject to confirmation bias; their reaction to empirical results highly depends on whether these results support their a priori postulated hypotheses? Databases constructed for this type of research primarily serve the purpose to address a 
particular (medical) problem. This type of research is concerned with establishing a statistical model that provides a good explanation of the associations observed in the data ${ }^{2}$. These traditional statistical analyses are therefore known as 'confirmatory analyses' ${ }^{10}$. In contrast, knowledge discovery techniques serve an open discovery process, aimed at discovering unexpected patterns, without a priori postulating hypotheses. Such techniques are therefore, in principle, exploratory and ad hoc. The goal is to find the unexpected, that is, to acquire knowledge that is not necessarily the result of observations made in earlier research. Such findings may generate new hypotheses and the search for associations between phenomena that were not previously thought of ${ }^{8}$. Knowledge discovery techniques are often applied to data that are collected without any predetermined purpose. The contents of these databases are, most often, dynamically changing (e.g. electronic patient records).

Because of the proliferation of data in the past decades, there are several statistical analyzing methods that are aimed at exploratory research, such as cluster analyses and decision trees. However, these traditional statistical methods are of limited value for exploratory research when applied to large datasets ${ }^{2}$; patterns in a scatterplot become difficult to interpret when containing thousands or even millions of data points ${ }^{2}$. Creating a correlation matrix of thousands or millions of variables is extremely time-consuming and perhaps even impossible when conducted by traditional statistical methods. Moreover, statistical methods are known to rely on a arbitrary division in "significant" or "nonsignificant" findings (i.e. it commonly employs a threshold of $\mathrm{P}=0.05$, i.e. a type I error of $5 \%)^{11}$. However, in a large dataset this employed strategy will find evidence for even the smallest effects. Consequently, chance findings will be published based on the criteria for statistical significance ${ }^{11}$. In sharp contrast, in the interactive KDD process the subjective judgment of the (medical) user is extremely relevant; the user (being the expert) has to decide if the discovered patterns indeed satisfy a priori stated criteria (i.e. predictive validity on new data, novelty of the patterns, clinical relevance, pragmatic utility) ${ }^{10}$. For example, the researcher should come up with a theoretical framework that explains the patterns that were found. Thus, instead of interpreting the data in terms of statistical significance (i.e. pvalues and confidence intervals), data mining needs more careful considerations: e.g. are the discovered patterns new, important and clinically valuable ${ }^{12}$ ? Furthermore, in contrast to KDD applications, statistical methods should adjust for confounding factors (i.e. factors that are related to the independent as well as the dependent variable). Since KDD is aimed at hypothesis generation rather than on explaining the patterns observed in the data, i.e. investigating whether A causes B, B causes A or an unknown factor that causes both, KDD application do not have to adjust for possible confounding factors.

\section{THE KDD PROCES}

Knowledge discovery incorporates a new generation of data analyses that has emerged from the need to identify meaningful patterns in sometimes massive amounts of data. The basic steps of the KDD process, as first introduced by Fayyad, Piatetsky-Shapiro and Smyth ${ }^{1}$, will be outlined here. An ideal system for KDD should support all these steps starting with raw data up to producing useful knowledge (see Figure 1$)^{13}$. Some steps in the KDD 
process are merged and simplified in order to maintain the conciseness and comprehensibility of this paper. Although many KDD applications are readily applicable, real-world databases present difficulties due to the nature of their contents which tend to be dynamic, incomplete, redundant and noisy ${ }^{14}$. Enhancing the consistency of the data will consequently improve the next steps of the KDD process ${ }^{15}$. This first step is known to be the most time consuming step of the KDD process. Data recorded using manual data entry can contain incorrect or imprecise data and may be the cause of malfunction and error ${ }^{13}$. Data cleaning and pre-processing is therefore an important and necessary preparatory step. This includes the detection and correction of inaccurate records including the removal of outliers and duplicate records and decisions on strategies how to handle missing data ${ }^{1}$.

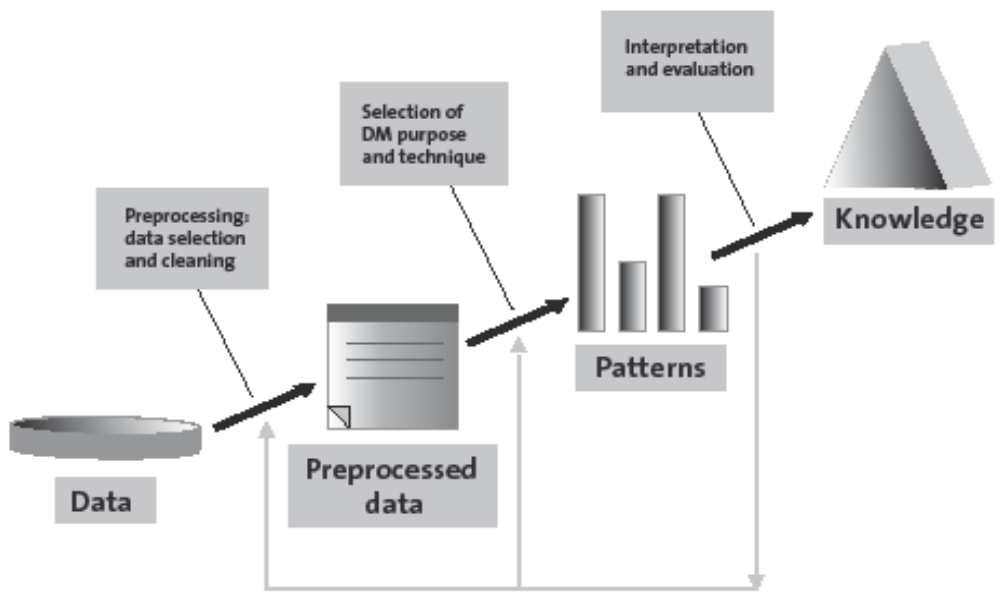

Figure 1. The KDD process (simplified) as firstly introduced by Fayyad, Piatetsky-Shapiro and Smyth (1996). Printed with permission from Piatetsky-Shapiro.

Redundant data or data irrelevant for the problem at hand must eliminated in order to simplify the task of discovering of hidden patterns in the datasets ${ }^{16}$. For example, data beyond the scope of the study, i.e. data on younger people when the study at hand concerns people aged 70 and older can be deleted without loss of information.

In order to achieve the primary goals of the researcher, a data-mining method, suitable for the database and research at hand must be found, which is the second step in the data mining process. Data mining has different purposes to give meaning to data, which include summarizing, classification, regression or clustering. Although these data mining purposes might appear different at first glance, they share many components and it is therefore not uncommon to find that they can be applied to the same analyzing problem ${ }^{1}$. For example, a study by Gregori and co-workers (2009) compared six, commonly used data mining methods to determine the covariates selection, predictive accuracy and clinical indication of analytical models for predicting mortality in patients with diabetes mellitus ${ }^{17}$. Using these 
new techniques the authors were able to conclude that their techniques did not severely differ in predictive capability (around 70\%) and that therefore multiple techniques can be used to analyze one and the same data set.

The third step involves the actual 'mining' of the data. Data mining is the process of discovering patterns in large databases in an automatic or semiautomatic manner ${ }^{1}$. This can, for example, result in new classification schemes, newly generated hypotheses or new predictive models which were previously unknown. For example, a data mining technique referred to as 'association rules' can, in contrast to traditional statistical methods, evaluate tens or even hundreds of different prognostic variables in order to predict disease outcome.

In the last, fourth step, the 'mined' patterns must be interpreted in a meaningful way in order to be presented as 'knowledge'. The patterns discovered by data mining should be meaningful and lead to some particular advantage. The knowledge of a domain expert is crucial when the found patterns are evaluated from a conceptual point of view. Since the judgment of the user is essential, the KDD process can be viewed as an interactive process between automated, data mining driven techniques and the expert in (bio)medical research or epidemiology. The user can decide if the discovered patterns indeed satisfy the a priori postulated criteria by evaluating the predictive validity of the data mining technique on the one hand, and the novelty, the pragmatic utility and the comprehensibility of the end results on the other ${ }^{18,19}$. For instance, the degree of concordance in predictive accuracy of a model when applied to new data may be an important criterion, since patterns and associations found in database A should also be present in database B when it contains the same type of information. This final step in the KDD process may involve visualization of the extracted patterns but can also, as can be seen from the figure below, include returning to previous steps in the KDD process if necessary ${ }^{19}$.

\section{KNOWLEDGE DISCOVERY TECHNIQUES}

Ever since KDD started to be recognized as a well-defined area of research, investigators have developed new techniques and tools for the automatic extraction of useful information ${ }^{20}$. KDD techniques can be subdivided into two primary approaches: directed data mining, also named supervised learning, and undirected data mining, also known as unsupervised learning ${ }^{6}$.

Directed or supervised data mining involves 'training' of a model in order to explain patterns in data in the most appropriate manner. Supervised data mining operates under 'supervision' by being provided with the actual outcome for each training example (e.g. risk/no risk group or frailty vs. successful aging). Directed or supervised data mining techniques include classification, prediction models (e.g. regression models) and association rules. Undirected data mining involves the search for similarities between objects in the data in order to determine how they can be characterized. Hence, no predetermined outcome measures are set. Neural networks, clustering and feature extraction techniques are examples of undirected or unsupervised data mining.

Roiger (2003) clarified the difference between supervised and unsupervised data mining by comparing classification methods to clustering methods ${ }^{21}$. For example, assume that a supervised model was conducted with a prior postulated dichotomous outcome 
measure called 'diseased yes/no' (i.e. diseased vs. healthy persons). The supervised data mining approach will use the available variables (e.g. genetics, and sociodemografic and pathophysiologic variables) to predict the outcome at hand. In contrast, unsupervised data mining will use the same variables in order to examine if the two clusters (i.e. diseased and healthy) are naturally formed without a predetermined outcome ${ }^{21}$. Hence, supervised classification models are conducted by forming concept definitions from sets of data containing predefined classes, while unsupervised clustering builds models from data without the aid of predefined classes. In the latter, data are grouped together based on a similarity scheme defined by the clustering method. Two examples of data mining techniques, association rule learning and classification, which both are highly usable for (medical) epidemiologic research, will be outlined hereafter.

\section{Association-Rules}

Association rule learning is one of the most popular data mining applications for discovering relations between variables. Association rules, firstly described by Agrawal (1993), are aimed at producing rules that can establish relationships between events by predicting the occurrence of one event based on the occurrence of another event ${ }^{22}$. An association rule makes use of the form $X \rightarrow Y$. Such a rule could be, for example, [high blood pressure + high cholesterol $] \rightarrow$ [cardiovascular disease], meaning a diagnosis of high blood pressure and high cholesterol encompasses an increased risk for cardiovascular disease. The primary aim of association rules is to find relationships that apply to a large number of items (e.g. patients) and have a high accuracy ${ }^{23}$. The best known and generally used criteria for association rules are support and confidence which will be explained hereafter, by using a simple database in clinical epidemiology.

Consider a small data table as displayed in Table 1. The support of an association rule is the ratio (in \%) of the instances for which it predicts correctly, often also called the coverage of a rule. Hence, support is the frequency of coexistence of certain attributes (i.e. variables).

Table 1. Example database including 4 medical conditions and 6 patients

\begin{tabular}{ccccc}
\hline$\#$ & High blood pressure & High cholesterol & $\begin{array}{c}\text { Pulmonary heart } \\
\text { disease }\end{array}$ & $\begin{array}{c}\text { Cerebrovascular } \\
\text { accident }\end{array}$ \\
\hline 1 & 1 & 1 & 0 & 0 \\
2 & 0 & 1 & 1 & 0 \\
3 & 1 & 1 & 1 & 0 \\
4 & 0 & 1 & 0 & 0 \\
5 & 0 & 0 & 0 & 1 \\
6 & 1 & 1 & 0 & 1 \\
\hline
\end{tabular}

Note: $0=$ diagnosis is absent and $1=$ diagnosis is present

In the previous example, [High blood pressure + high cholesterol] has a support of $3 / 6=$ 0.5 since this combination occurs in $50 \%$ of all patients. In general, rules with large support are preferred over rules with low support. For example, clinical epidemiologists may be more concerned about how two (or more) medical conditions are related if they 
occur in $20 \%$ of all patients than a combination that occurs in only $2 \%$ of all patients. However, while a high support often is desirable, this is not always the case. Even if a combination of medical conditions does not occur very frequently, association rules showing a relationship between two medical conditions that has serious consequences (e.g. pneumonia in people with Chronic Obstructive Pulmonary Disorder (COPD)) is highly important.

A second important requirement is confidence. The Confidence of a rule is the ratio (in \%) of the instances that is predicted correctly, expressed as a proportion of all instances it applies to. Confidence indicates the degree of correlation in the dataset between $\mathrm{X}$ and $\mathrm{Y}$ and is therefore a measure of the strength of a rule22. For example, the rule [high blood pressure + high cholesterol $] \rightarrow$ [pulmonary heart disease] has a confidence of $0.17 / 0.5=0.33$. This means that $33 \%$ of patients, diagnosed with both high blood pressure and high cholesterol, will be diagnosed with pulmonary heart disease.

In general, a minimum support and confidence threshold are specified before the data mining will be applied. Hence, the mining of association rules from a database implies to find all rules that meet the specified threshold for support and confidence ${ }^{22}$. The previous example illustrates that association rules are not restricted to establishing relationships between two attributes. Thus, many different association rules, predicting various different outcomes (e.g. pulmonary heart disease or cerebrovascular accident) can be derived from even a small dataset with a limited amount of attributes, which we will explain below.

A study conducted by Ordonez (2006) was aimed at relating perfusion measurements and risk factor to vessel disease to improve actual diagnosis. The dataset contained 655 patients and 25 medical attributes, including personal information (e.g. age, a diagnosis of diabetes or prior stroke and smoking habits) and medical measurements (e.g. weight, heart rate, blood pressure). In contrast to traditional statistical techniques, association rules are able to evaluate 25 different variables and their elaborative interactions. The techniques produced 771 association rules related to healthy arteries and 522 rules related to diseases. Some rules were expected (i.e. low age, female sex and low cholesterol levels appeared to be related to healthy arteries), which confirmed the validity of the technique. Other results were more surprising; a defect in a specific heart region (Septo-Anterior) relates positively with a chance of having a diseased Left Anterior Descending artery ${ }^{24}$. This study concluded that association rules provide an alternative for statistical techniques when many possible predictive factors are available. Moreover, although data mining can evaluate tens or even hundreds of attributes, this example shows that it is also possible to generate clinically relevant information from a small number of attributes in a small data set.

Association rules can be applied to a large variety of problems since each association rule represents a different kind of knowledge. Association rules can, for example, predict drug prescription given a certain disease combination and such a priori knowledge could subsequently be used to prevent adverse drug interactions. Furthermore, as illustrated by the abovementioned example, association rules can also predict future diagnoses of diseases based on already present risk factors. Association rules can thereby promote early detection and possibly even prevention of diseases. 


\section{Classification}

Classification is probably the most widely used data mining technique and aims to explain data by predicting the classification of objects (e.g. patients) into the correct class (e.g. being at risk yes/no $)^{25}$. Its primary goal is to divide the dataset into mutually exclusive classes such that the units within each class are as "close" as possible to one another, and the units between different classes are as "far" as possible from one another. The difficulty is to define classifiers (also known as attributes or variables) that serve as decision markers for classifying the data appropriately and in a meaningful manner.

In order to conduct a classification task the sample at hand needs to be divided into two independent representative samples of the original data sets: a training and a test $\operatorname{set}^{23}$. The training set is used to construct the classification model, i.e. to learn how to classify the data appropriately. The performance of this model can be measured in terms of an error rate: the proportion incorrectly classified objects in the data set. In order to predict the performance and utility of the classification model on new data, and thereby assess the 'goodness-of-fit' of the model, the error rate needs to be checked on a dataset that was not used to construct the actual model ${ }^{23}$. This independent dataset is called the test set. A test set is a set of data that is independent of the training data, but that follows the same probability distribution as the training data. Hence, the test set is inevitable for the evaluation of the model conducted by making use of the training set. The larger the test sample, the more accurate the error estimate will be. Some models, conducted based on the training data, tend to 'overfit' the data; some relationships, identified in the training data, do not hold in general (i.e. in other databases). If a certain model fits both the training and the test set well, minimal 'overfitting' has taken place.

Besides predicting accuracy of the model on new data, the ecological validity of the classification model is also very important. For example, to support a medical doctor in his daily routine, a model needs to be able to support doctors' decisions ${ }^{25}$. Typically, the more complex models may fit the data better but may also be more difficult to interpret ${ }^{1}$. In some cases a third data set, the validation set, is necessary in order fine-tune the model. Of course, all data sets have to be representative samples of the data at hand. By using similar data for training and testing, one can minimize the effects of data discrepancies and better understand the characteristics of the constructed model.

If enough data are available, splitting the data into two or even three independent test sets is a useful option. The division of the data into a training and test set when only a limited amount of data is available might lead to biased results, since the error rate is expected to remain large. A k-fold cross-validation can maximize the use of the training and test data. The data can be divided into $\mathrm{k}$ subsets of equal size (normally $\mathrm{k}=10$ ). The training set will include k-1 subsets, while one subset is used for testing. This process then can be repeated $\mathrm{k}$ times so that all subsets are used once as a testing set. The average performance on the k subsets can then be computed.

In a study conducted by Bae and colleagues, decision trees, a type of classification method, were used to predict good social functioning in schizophrenia patients ${ }^{26}$. A decision tree is a tree structure that makes use of decision rules to predict class partnership. Non-terminal nodes in a decision tree represent decisions on one or more classifiers and 
terminal nodes reflect decision outcomes. The advantage of using decision trees is that it can include all possible variable combinations into one decision tree. This study included 67 schizophrenia patients and a total of 51 variables including data on demographic characteristics, medical history, social cognition and neurocognitive and psychiatric symptoms. The aim of the study was to integrate these possible factors in order to find relationships among variables that were not previously considered. The authors decided to make use of decision trees, since analyzing 51 variables while only 67 subjects are included made the use of traditional statistical techniques unfeasible. By integrating so many possibly relevant predictive factors, the authors concluded that the best social functioning can be predicted by the combination of three variables: good sustained attention, good Theory Of Mind performance and low sensitivity for negative emotions ${ }^{26}$.

Another study, conducted by Panzarasa and colleagues (2010), investigated if compliance with clinical guidelines improved health in patients who recently had a stroke ${ }^{27}$. The primary aim of the study was to assess which individual health recommendations improve health outcomes in 5.079 stroke patients, by making use of classification trees. In contrast to their expectations, they found that graded compression stockings as well as pneumatic compression did not benefit the health of patients with a severe initial stroke status. This result was in accordance with results from a randomized controlled trail conducted by Dennis and colleagues, which raised doubts about such prevention procedures $^{28}$.

\section{TEXT MINING}

To date, we are not only confronted with data gathered in medical databases but also with a vast body of information published in medical literature. MEDLINE, the main repository of published (bio)medical literature, and therefore widely used among medical researchers, contains references to medical, nursing, dental, veterinary and health care journal articles that were published since 1948. It incorporates over 18 million citations (i.e. a reference to a published source), including links to full text articles and other related resources, and is growing at a rate of 500.000 citations each year.

Although text is the predominant medium for information exchange among (medical) experts $^{29}$, it has become impossible for medical researchers, health policy makers and medical doctors to keep abreast with all the publications. This abundance in available literature may be so overwhelming that they may soon inhibit rather than stimulate scientific progress ${ }^{30}$. However, all these medical articles, even those beyond the direct scope of the researcher, may contain valuable information related to medical research and clinical applications.

Increasing amounts of information and observations, recorded in medical research articles, are derived from different, apparently unrelated areas of research (e.g. genetic research, epidemiology, primary care research). Useful implications of scientific discoveries can go unnoticed or unutilized because they exist only implicitly in scattered information in different areas of research. However, they can contribute to a greater understanding when considered collectively ${ }^{31}$. 
To use the increase of scientific publications to its full extent, text mining techniques have been introduced that can support the scientific reader with automatic tools to investigate substantial amounts of text. Text mining involves analyzing large collections of documents to discover promising new hypotheses ${ }^{32,33}$. This information might involve relationships or associations that are hidden in (collections of) documents that would otherwise remain undiscovered. Text mining, as well as, data mining, is a highly interactive and user-involved procedure. Domain experts are needed to evaluate the validity and usability of the newly generated hypotheses.

Text mining discovers and extracts knowledge from literature in three consecutive steps. First, information retrieval takes place in order to identify those documents that match a user's query. This step is aimed at reducing the number of documents for analysis, by applying computational algorithms to large collections of documents (i.e. Medline). Secondly, information is extracted from these selected documents. Information extraction is the automatic process of obtaining structured data from an unstructured text document. Information extraction selects specific facts about pre-specified entities (e.g. genes, proteins, medical conditions) that are available in the documents ${ }^{34}$. Most efforts in medical information extraction are devoted to 'term analysis'; the identification of terms denoting specific classes of (bio)medical entities. In the last step of the text mining process the actual knowledge discovery takes place, i.e. specific relations between the extracted pieces of information are screened for (e.g. protein-protein interactions or disease combinations). The idea behind text mining is to relate or associate entities to each other based on their associated sets of texts ${ }^{35}$. Text mining approaches typically rely on the occurrence or cooccurrence of terms and have been successfully applied to a number of problems. These entities can be investigated for hidden patterns or can be classified or clustered. Consequently, implicit knowledge can be detected, which may result in well-motivated and testable hypotheses 5 .

There are a variety of automatic text mining approaches to infer implicit knowledge from literature databases. One of these approaches is Swanson's ABC model (see Figure 2) which is commonly described as the possibility to link different scientific disciplines through intermediate or shared interests ${ }^{36}$.

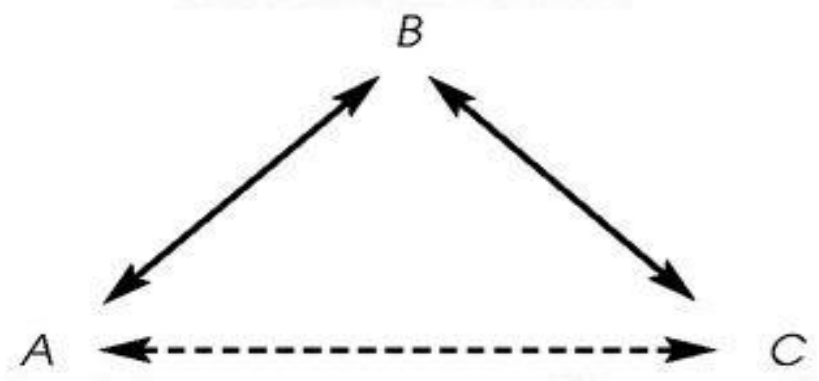

Figure 2. Swanson's ABC model 
Start with object A (i.e. a chronic disease). We can identify other object(s) B (i.e. cooccurring chronic diseases) that are related to A within the (medical) literature. By identifying the co-occurrence of $\mathrm{A}$ and $\mathrm{B}$ within Medline records, the relationship between $A$ and $B$ can be established. Similarly, each object B can then be queried to identify other objects like $\mathrm{C}$, which co-occurred with $\mathrm{B}$, but not with $\mathrm{A}$, within the titles or abstracts of articles within Medline. Thus, every object $\mathrm{C}$ is only implicitly related to $\mathrm{A}$, since $\mathrm{A}$ and $\mathrm{C}$ are not mentioned within the same title or abstract. They only share one or more relationship with B. This list of implicit relationships may lead to potential discoveries of new relationships.

Swanson and co-workers were the first to demonstrate that text mining, by making use of Medline, can lead to the discovery of new knowledge. They used Medline to make connections between seemingly unrelated entities, in this case the connection between migraine and magnesium deficiency ${ }^{36}$. Swanson and co-workers were also able to identify a relationship between Reynaud's disease and the dietary effects of fish oil, which led to hypothesis and subsequent proof that compounds within dietary fish oil could ameliorate the symptoms of Raynaud's disease ${ }^{37}$. Swanson's ABC approach has also been applied to other areas of medicine resulting in new relationships between medical conditions ${ }^{38}$ and the generation of new therapeutic indications of pharmaceuticals ${ }^{39}$.

\section{CONCLUSIONS}

The rapid development of tools for acquisition and storage of information has lead to vast medical databases with large amounts of medical records as well as vast amounts of available medical literature. Medical databases tend to increase in both the number of $\mathrm{N}$ records (e.g. patients) as well as in the number of fields, or variables ${ }^{19}$. The large medical datasets as well as the large body of medical literature can be potentially relevant in the context of dedicated medical research questions and can, eventually, lead to the improvement of health care. Nevertheless, medical researchers, health policy makers and medical doctors have not yet taken full advantage of the potential to effectively extract useful information from these enormous amounts of data and text.

Although some traditional statistical methods are also aimed at hypotheses generation, KDD approaches tend to be far more suited for exploratory research ${ }^{40}$. Knowledge discovery approaches are not intended to replace the statistical analyzing techniques, but can be of use in the practice of hypotheses generation. Of course, these hypotheses still need further evaluation and validation using in-depth research. Hence, KDD merely extends current statistical methods by taking advantage of technology and the development in research areas, such as computational power, artificial intelligence and machine learning. Moreover, when using KDD, researchers have no or limited conformation biases since no a priori hypotheses are postulated.

As is the case with all analyzing methods, uncritical appraisal of implementation of data and text mining applications (critized as 'data dredging' or 'fishing expeditions' ${ }^{\text {' }}$ ) can lead to the discovery of erroneously, meaningless or invalid patterns. Therefore, medical researchers confronted with large amounts of medical data and/or medical documents are recommended to consult an expert in the field before using, interpreting and reporting on 
these KDD applications. Knowledge discovery typically combines automated mining approaches, as the nature of data and text mining methods imply, with medical user interaction to ensure accurate, useful, and understandable results. Human involvement and knowledge and expertise is needed to evaluate if the discovered knowledge is of value to the medical researcher ${ }^{40}$. Rather than to serve as a golden standard, the application of data and text mining in the medical domain is primarily aimed at supporting a specialist's decisions. The objective of the present paper was to introduce and to give a short background on knowledge discovery in databases. Given the broad spectrum of knowledge discovery, only a snapshot could be presented here. Consequently, a direct extension of this work is needed to make researchers, who are already familiar with statistical analyzing methods, more acquainted with other analyzing methods that knowledge discovery in databases can provide. Moreover, more information related to the implementation of these methods should be available in order to support researchers in the actual use of data and text mining.

In conclusion, knowledge discovery refers to a broad process of finding implicit knowledge that can only be inferred by data mining and text mining tools integrated in the practice of hypothesis-driven health research. Although knowledge discovery is not a universal solution, it has proven to be useful tool that has the potential to expand our knowledge by opening up hidden sources of knowledge contained in medical data ${ }^{6}$. 


\section{REFERENCES}

1. U. Fayyad, G. Piatetsky-Shapiro, and P. Smyth. From Data Mining to Knowledge Discovery in Databases. Artificial Intelligence Magazine, 1996;17(3):37-54.

2. J.D. Hand. Statistics and Data Mining: Intersecting Disciplines. ACM SIGKDD Explorations Newsletter, 1999;1(1):16-19.

3. R. Harpaz, H.S. Chase, and C. Friedman. Mining multi-item drug adverse effect associations in spontaneous reporting systems. BMC bioinformatics, 2010;11(9).

4. C. Piatetsky-Shapiro. Knowledge Discovery in Real Databases: A Report on the IJCAI-89 Workshop. Artificial Intelligence Magazine, 1991;11(5):68-70.

5. M. Weeber, J.A. Kors, and B. Mons. Online tools to support literature-based discovery in the life sciences. Brief Bioinform., 2005;6(3):277-286.

6. A.M. Berger and C.R. Berger. Data mining as a tool for research and knowledge development in nursing. Computers, Informatics, Nursing, 2004;22(3):123-131.

7. W.R. Swindell, K.E. Ensrud, P.M. Cawthon, J.A. Cauley, S.R. Cummings, R.A. Miller, and S.O. Sof. Indicators of "Healthy Aging" in older women (65-69 years of age). A data-mining approach based on prediction of long-term survival. BMC Geriatrics, 2010;10(1):55.

8. J.H. van Bemmel, E.M. van Mulligen, B. Mons, M. van Wijk, J.A. Kors, and J. van der Lei. Databases for knowledge discovery. Examples from biomedicine and health care. Int. J. Med. Inform., 2006;75(3-4):257-267.

9. M. Bakker and J.M. Wicherts. The (mis)reporting of statistical results in psychology journals. Behav. Res. Methods, 2011.

10. H. Mannila. Data Mining: Machine Learning, Statistics, and Databases. SSDBM. 1996:2-9.

11. J.A. Sterne and G. Davey Smith. Sifting the evidence-what's wrong with significance tests? BMJ, 2001;322(7280):226-231.

12. J.D. Hand. Data mining: statistics and more? The American Statistician, 1998;52:112-118.

13. B. Dunkel, N. Soparkar, J. Szaro, and R. Uthurusamy. Systems for KDD: From Concepts to Practice. Future Generation Computer Systems archive, 1997;13(2-3):231-242.

14. W. Matheus, P.K. Chan, and G. Piatetsky-Shapiro. Systems in Knowledge Discovery in Databases. IEEE Transactions on Knowledge and. Data Engineering, 1993;5(6):903-913.

15. X. Wu and X. Zhu, Mining With Noise Knowledge: Error-Aware Data Mining, in Proceedings of the 2006 IEEE International Conference on Granular Computing (GRC). 2006: Atlanta.

16. P. Wright. Knowledge discovery in databases: tools and techniques. Crossroads, 1998;5(2):23-26.

17. D. Gregori, M. Petrinco, S. Bo, R. Rosato, E. Pagano, P. Berchialla, and F. Merletti. Using Data Mining Techniques in Monitoring Diabetes Care. The Simpler the Better? J. Med. Syst., 2009.

18. I. Askira-Gelman, Knowledege Discovery: Comprehensibility of the Results, in Hawaii International Conference on System Sciences (HICSS), Society I.C., Editor. 1998: Kohala Coast, Hawaii, USA. p. 247-255.

19. U.M. Fayyad, G. Piatetsky-Shapiro, and P. Smyth. From Data Mining to Knowledge Discovery: An Overview, in AKDDM, AAAI/MIT, 1996;1-30.

20. J. Fürnkranz. A Brief Introduction to Knowledge Discovery in Databases. ÖGAI-Journal, 1995;14(4):14-17.

21. R. Roiger and M. Geatz. Data Mining: A tutorial Based Primer. 2002: Pearson Educational Australia.

22. R. Agrawal, T. Imielinski, and A. Swami (1993) Mining Association Rules Between Sets of items in Large Databases. SIGMOD Conference, 207-216.

23. I.H. Witten and E. Frank. Data mining: practical machine learning tools and techniques. 2nd. ed. 2005, San Francisco: Elsevier.

24. C. Ordonez. Association rule discovery with the train and test approach for heart disease prediction. IEEE Trans. Inf. Technol. Biomed., 2006;10(2):334-343.

25. M. Kukar, I. Kononenko, and T. Silvester. Machine learning in prognosis of the femoral neck fracture recovery. Artif. Intell. Med., 1996;8(5). 
26. S.M. Bae, S.H. Lee, Y.M. Park, M.H. Hyun, and H. Yoon. Predictive factors of social functioning in patients with schizophrenia: exploration for the best combination of variables using data mining. Psychiatry Investigation, 2010;7(2):93-101.

27. S. Panzarasa, S. Quaglini, L. Sacchi, A. Cavallini, G. Micieli, and M. Stefanelli. Data mining techniques for analyzing stroke care processes. Stud. Health Technol. Inform., 2010;160(2):939-943.

28. M. Dennis, P.A. Sandercock, J. Reid, C. Graham, G. Murray, G. Venables, A. Rudd, and G. Bowler. Effectiveness of thigh-length graduated compression stockings to reduce the risk of deep vein thrombosis after stroke (CLOTS trial 1): a multicentre, randomised controlled trial. Lancet, 2009;373(9679):1958-1965.

29. I. Spasic, S. Ananiadou, J. McNaught, and A. Kumar. Text mining and ontologies in biomedicine: making sense of raw text. Brief. Bioinform., 2005;6(3):239-251.

30. M. Weeber, R. Vos, H. Klein, L.T. De Jong-Van Den Berg, A.R. Aronson, and G. Molema. Generating hypotheses by discovering implicit associations in the literature: a case report of a search for new potential therapeutic uses for thalidomide. J. Am. Med. Inform. Assoc., 2003;10(3):252-259.

31. J.D. Wren. The emerging in-silico scientist: how text-based bioinformatics is bridging biology and artificial intelligence. IEEE Eng. Med. Biol. Mag., 2004;23(2):87-93.

32. M. Weeber, R. Vos, H. Klein, L.T. De Jong-Van Den Berg, A.R. Aronson, and G. Molema. Generating hypotheses by discovering implicit associations in the literature: a case report of a search for new potential therapeutic uses for thalidomide. J. Am. Med. Inform. Assoc., 2003;10(3):252-259.

33. P. Srinivasan and B. Libbus. Mining MEDLINE for implicit links between dietary substances and diseases. Bioinformatics, 2004;20(1):290-296.

34. I. Spasic, S. Ananiadou, J. McNaught, and A. Kumar. Text mining and ontologies in biomedicine: making sense of raw text. Brief. Bioinform., 2005;6(3):239-251.

35. A.R. Aronson. The effect of textual variation on concept based information retrieval. Proc. AMIA Ann. Fall. Symp., 1996:373-377.

36. D.R. Swanson. Migraine and magnesium: eleven neglected connections. Perspect. Biol. Med., 1998;31:526-557.

37. D.R. Swanson, N.R. Smalheiser, and V.I. Torvik. Ranking Indirect Directions in Literature-Based Discovery: The Role of Medical Subject Heading. J. Am. Soc. Inf. Sci. Technol., 2006;57(111): 14271439.

38. K.M. Hettne, A.J. Williams, E.M. van Mulligen, J. Kleinjans, V. Tkachenko, and J.A. Kors. Automatic vs. manual curation of a multi-source chemical dictionary: the impact on text mining. J. Chem. Inf. Model., 2010;2(1).

39. A. Waagmeester, P. Pezik, S. Coort, F. Tourniaire, C. Evelo, and D. Rebholz-Schuhmann. Pathway enrichment based on text mining and its validation on carotenoid and vitamin A metabolism. Omics, 2009;13(5):367-379.

40. S.P. Imberman. Effective use of the KDD process and data mining for computer performance professionals. Journal of Computing Resources, 2002;(107):68-77. 


\section{CHAPTER 8}

Patterns of multimorbidity: identifying psychiatric and somatic disease combinations using an exploratory analyzing approach

S. Aarts, R. Vos, M.P.J. van Boxtel, F.R.J. Verhey, J.F.M. Metsemakers and M. van den Akker. 


\begin{abstract}
Background

Multimorbidity, the co-occurrence of two or more chronic medical conditions within a single individual, is a highly prevalent health problem. A thorough understanding of frequently co-occurring medical conditions could improve health care delivery for individuals with multiple pathology. This study was aimed at identifying novel, potentially relevant psychiatric and somatic disease combinations using a knowledge discovery approach.
\end{abstract}

\title{
Methods
}

We focused on disease combinations with a prevalence of $\geq 10$ in a large general practice database (The Registration Network Family Practices; $N=87,837$ ) and an observed/expected ratio of $\geq 1.5$. Six conditions from the ICPC P (psychological) chapter were involved: affective psychosis (P73), depressive disorder (P76), schizophrenia (P72), mental retardation (P85), personality disorder (P80) and suicide attempt (P77). The following ICPC chapters with somatic conditions were used: D (Digestive), $\mathrm{K}$ (Circulatory), L (Musculoskeletal), N (Neurological), R (Respiratory) and T (Endocrine, metabolic and nutritional). In total, 166 different disease combinations (i.e. a combination of one psychiatric and one somatic condition) were selected. Observed/expected ratio's, odds ratio's and corresponding confidence intervals were calculated. An expert evaluation phase was employed to evaluate and discuss several disease combinations in more detail.

\section{Results}

In total, 37of the 166 disease combinations passed the prevalence and observed/expected ratio threshold and were evaluated by three medical experts. The following five disease combinations representing the broad scope of interaction between psychiatric and somatic disorders were discussed in detail during an expert meeting; appendicitis and personality disorder, uncomplicated hypertension and psychotic affective disorders, migraine disorder and personality disorder, pulmonary emphysema and depressive disorder, and lipid metabolism and depressive disorder.

\section{Conclusions}

Using a knowledge discovery approach, in which two selection criteria were combined with an expert evaluation phase, the present study identified several undiscovered and potential relevant disease combinations in a large general practice data set. These disease combinations need to be confirmed in other, preferable general practice based, datasets. In addition, the findings indicate that hypothesis-driven research using general practice based data is able to generate novel medical information. 


\section{INTRODUCTION}

Multimorbidity, the presence of two or more co-occurring diseases within one person ${ }^{1}$, is not evenly distributed among individuals: while some individuals remain healthy their entire life, others get one disease after the other. Patterns of multimorbidity, i.e. a specific combination of two or more chronic conditions, might co-occur if conditions cause, precipitate, or maintain one another directly or indirectly ${ }^{2}$. Psychiatric and somatic disease combinations have a tendency to co-occur ${ }^{2}, 3$. Co-occurring psychiatric and somatic conditions are well-known for their adverse health effects; besides causing distress and functional disability, comorbid psychiatric illnesses are also related to non-adherence of self-care (e.g. improper use of medication) and prolonged recovery time ${ }^{4}$. Moreover, patients suffering from multiple medical conditions use health care services more frequently and use a greater array of health services than other consumers of care 5 . Because of the increase in multimorbidity, general practitioners are increasingly confronted with complex health care situations of patients with co-occurring conditions especially since one condition might influence and even exacerbate the other conditions $(s)^{6}$.

Research suggests that some chronic conditions co-occur more frequently than would be expected when these conditions were statistically independent. Several psychiatric and somatic disease combinations such as depressive disorder in individuals with diabetes mellitus type $\mathrm{II}^{7}$ and metabolic syndrome in individuals with schizophrenia ${ }^{8}$, have already been established. Nevertheless, given the large spectrum of chronic psychiatric and somatic conditions more clusters of co-occurring psychiatric and somatic conditions are anticipated. However, if we want to investigate all possible existing combinations of co-occurring chronic conditions we face an enormous amount of possible disease combinations. Hence, with exception of some studies 9,10 , researchers have avoided these difficulties by studying only on a limited number of frequently occurring chronic conditions ${ }^{11}$ or by selecting an index disease ${ }^{12}$. One way to cope with this overload of medical information is by means of knowledge discovery approaches ${ }^{13}$, which incorporates a new generation of data approaches including data mining. These approaches have emerged from the need to identify meaningful patterns in large amounts of $\mathrm{data}^{14-16}$ and are aimed at finding the unexpected, that is, to discover associations and patterns that do not logically follow from earlier research ${ }^{17,18}$. These exploratory methods offer significant advantages over more traditional analyzing techniques, not in the least because of increased computational power ${ }^{14,19}$.

We hypothesized that a knowledge discovery approach will be able to analyze a large general practice based data set in order to successfully identify novel (i.e. not previously considered in the medical field), potentially relevant (i.e. useful in the practice of health care) combinations of psychiatric and somatic conditions. Knowledge regarding frequently occurring disease combinations can improve health care delivery for individuals with multimorbidity by, for example, improving our ability to predict and diagnose co-occurring medical conditions and adjust health care delivery for individuals with specific disease combinations. 


\section{METHODS}

\section{Study Population}

Data stems from the Registration Network Family Practices (RegistratieNet Huisartspraktijken, RNH) available on July $1^{\text {st }}, 2010$ and consisted of 87,837 individuals of all ages. The $\mathrm{RNH}$ is a continuously updated database, in which 70 general practitioners (GPs) working in 22 family practices in the south of the Netherlands are participating. This database includes all relevant current and past health problems. A health problem is defined as 'anything that has required, does or may require health care management and has affected or could significantly affect a person's physical or emotional well-being ${ }^{20}$. In general, health problems are only corded by the GP when they are permanent (no recovery expected), chronic (duration longer than 6 months) or recurrent (more than three recurrences within 6 months), or when they have lasting consequences for the functional status or prognosis of the patient. These problems are coded in a standardized fashion, according to the International Classification of Primary Care (ICPC) and other more current guidelines of the Dutch College of GP's. ICPC is a diagnostic classification developed under the umbrella of Wonca and the WHO, with relations to other disease classifications such as the $\mathrm{ICD}^{21}$. In complex medical conditions registration is almost always based on a specialist diagnosis reported to the GP.

This database contains background information on the patient's sex, date of birth, marital status, type of household, code of practice and, level of education. Membership of the RNH population ends by migration or death. All patients included in the RNH database have been informed about the anonymous use of their health information and their medical information is not incorporated in the database if desired. The quality of the data is ascertained by ample instruction and training sessions, regular regional consensus groups, quality control audits, an online thesaurus available during data-entry and systematic control ${ }^{22-24}$. The present study population was comparable to the Dutch population with regard to socio-demographic variables ${ }^{25}$. This renders the $\mathrm{RNH}$ a valid and precise database for medical research.

\section{Included Chronic Diseases}

In order to limit the number of disease combinations, the following six conditions, coded in ICPC chapter P (Psychological), were involved: affective psychosis (P73), depressive disorder (P76), schizophrenia (P72), mental retardation (P85), personality disorder (P80) and suicide attempt (P77). Four of these conditions (i.e. affective psychosis, depressive disorder, schizophrenia and personality disorder) need to be considered severe psychiatric disorders (in contrast to mental retardation and suicide attempt). Although not all six conditions can be defined as psychiatric conditions, in order to preserve the comprehensibility of the present article, the term 'psychiatric' will be used throughout the manuscript to refer to the abovementioned six conditions.

The following ICPC chapters with somatic conditions were included: D (Gastrointestinal), K (Circulatory), L (Musculoskeletal), N (Neurological), R (Respiratory) and $\mathrm{T}$ (Endocrine, metabolic and nutritional). Only lifetime prevalences of diagnostic 
ICPC codes were included. ICPC codes representing symptoms and complaints were not selected even as test results not leading to a diagnosis, disease absence, deviations of normal function, superficial injuries, and risk factors. In total, 166 different disease combinations, which consisted of one psychiatric (i.e. ICPC chapter P) and somatic condition (i.e. ICPC chapter D, K, L, N, R and T), were selected for evaluation.

\section{Selection Criteria}

In order to select potentially relevant disease combinations two selection criteria with corresponding thresholds were employed. Firstly, disease combinations that are relatively prevalent among patients were selected, i.e. only disease combinations with a prevalence of $\geq 10$ (i.e. 10 individuals or more) in the RNH were selected. Secondly, Observed/Expected ratio's (O/E ratio's) were calculated. The observed probability of a specific combination of two diseases is more meaningful when compared to the expected probability ${ }^{26,27}$, since it can filter out disease combinations that are likely to follow from mere chance alone. The $\mathrm{O} / \mathrm{E}$ ratio is a measure of strength between two diseases; it allows disease combinations to be classified as positively associated, negatively associated, or not associated ${ }^{10}$. Calculation of the probability of two co-occurring diseases is based on the assumption of statistical independence (i.e. the two diseased co-occur at random) of the diseases concerned. An O/E ratio with value 1 indicates that the observed proportion of patients with a specific diseases combination equals the expected proportion. Hence, if the observed and expected probabilities are equal, a combination of diseases more likely will be independent of one another. Disease combinations with an $\mathrm{O} / \mathrm{E}$ ratio of 1.5 (i.e. the disease combinations occurs $50 \%$ more frequently as would be expected given the prevalence estimates of the two diseases) or more was considered statistically dependent ${ }^{9}$.

When evaluating associations between a maximum of two disorders, patterns of frequently occurring disease combinations are also commonly addressed by Odds Ratio's $(\mathrm{ORs})^{2,28}$. Compared to O/E ratio's, which suffer from several conceptual problems (e.g. lack of symmetry), ORs provide a more natural measure of performance ${ }^{29}$. ORs are defined as the ratio of the odds of an event (e.g. a psychiatric condition) to the odds of another event (e.g. a somatic condition) ${ }^{30}$. For 57 potentially relevant psychiatric and somatic disease combinations (i.e. combinations with a high prevalence and a high $\mathrm{O} / \mathrm{E}$ ratio), ORs and corresponding $95 \%$ confidence intervals (CIs), based on the logarithm of the odds were calculated ${ }^{29}$.

\section{Expert Evaluation}

The expert evaluation included three phases. The first phase consisted of the evaluation of the 166 disease combinations by one expert. The second phase required the evaluation of a selection hereof by three experts. The third phase involved an expert meeting in which several disease combinations were discussed. In the first and second phase, the experts were instructed to evaluate disease combinations with one of the following ratings: 0) no causal relation; 1) causal relation possible; 2) causal relation expected; and 3) otherwise related (e.g. as epiphenomenon or as a result of the diagnostic process). The experts were not allowed to use any knowledge resources to base their evaluation upon. For the third expert 
phase, a discussion among experts in an expert meeting, five potential relevant disease combinations were selected as examples of the large set of psychiatric and somatic disease combinations. Every disease combination was discussed in 5 to 10 minutes. The three experts were asked to bring forward their thoughts, ideas and reasoning schemes behind their evaluation. This expert meeting comprised a brainstorming type 31,32 ; this meeting was not intended to achieve consensus among the three experts but was aimed at evoking the thoughts and views behind the experts' evaluations regarding the disease combinations.

\section{RESULTS}

\section{Descriptive Characteristics}

Descriptive characteristics of the general practice-based population sample from $\mathrm{RNH}$ are reported in Table 1 .

While $19.7 \%$ had none of the included diseases, $16.3 \%$ of the participants were diagnosed with one medical condition and $64 \%$ of the study sample was diagnosed with multimorbidity (i.e. two or more chronic conditions present). In the 87,837 individuals, 302,808 chronic conditions were diagnosed, including 13,384 psychiatric disorders. In total, $10,807(12.3 \%)$ of the participants reported one or more psychiatric disorders; somatic conditions were present in 69,798 (79.5\%) participants.

\section{Disease Combinations Passing the Selection Criteria}

Table 2 displays the number of disease combination in relation to the two selection criteria, i.e. a prevalence of $10 \geq$ and a $\mathrm{O} / \mathrm{E}$ ratio of $\geq 1.50$. Of the 116 disease combinations, 54 (46.6\%) disease combinations passed the threshold of the two postulated criteria.

\section{Expert Evaluation}

The first step of the expert evaluation phase required the evaluation of the 166 disease combinations by a psychiatrist/scientific research in the field of psychiatry and neuropsychology. Table 3 shows the relation between the two postulated selection criteria and this expert evaluation.

Of the 166 disease combinations, $6.0 \%(n=10)$ were evaluated as 'causal relation possible', $1.8 \%(\mathrm{n}=3)$ were evaluated as 'causal relation expected', and $12.0 \%(\mathrm{n}=20)$ of the disease combinations were evaluated as 'related as epiphenomenon'. Moreover, $80.1 \%$ $(n=133)$ of the disease combinations were evaluated as 'no causal relation expected'. Note that disease combinations are only evaluated as 'causal relation possible', 'causal relation expected' and 'otherwise related', if they have a high $\mathrm{O} / \mathrm{E}$ ratio and a relatively high prevalence. In contrast, disease combinations with a low $\mathrm{O} / \mathrm{E}$ ratio and a low prevalence are also evaluated as 'no causal relation'. Hence, in general, medical conditions within a disease combination with a high prevalence and a high $\mathrm{O} / \mathrm{E}$ are known to be (causally) related. Moreover, the medical conditions in the majority of disease combinations with a low $\mathrm{O} / \mathrm{E}$ ratio and low prevalence are not known for their (causal) relation. However, as can be seen in table 3, there are also several disease combinations with a high prevalence and a high $\mathrm{O} / \mathrm{E}$ ratio which are evaluated as 'no causal relation'. This indicates that although these 
disease combinations are prevalent and have a high $\mathrm{O} / \mathrm{E}$ ratio, the expert is not aware of a causal relation. Therefore, these disease combinations might be novel for domain experts (i.e. experts in the field of psychiatry).

Table 1. Descriptive characteristics of the general practice-based population from $\mathrm{RNH}(\mathrm{N}=87,837)$

\begin{tabular}{|c|c|}
\hline Characteristics & Number of participants (\%) \\
\hline Gender (\% females) & $44,996(51.2)$ \\
\hline \multicolumn{2}{|l|}{ Age } \\
\hline $0-19$ & $16,137(18.4)$ \\
\hline $20-39$ & $18,937(21.6)$ \\
\hline $40-59$ & $28,342(32.3)$ \\
\hline $60-79$ & $20,250(23.1)$ \\
\hline$\geq 80$ & $4,171(4.7)$ \\
\hline \multicolumn{2}{|c|}{ Number of chronic conditions } \\
\hline 0 & $17,273(19.7)$ \\
\hline 1 & $14,328(16.3)$ \\
\hline 2 & $12,762(14.5)$ \\
\hline 3 & $10,350(11.8)$ \\
\hline 4 & $8,056(9.2)$ \\
\hline 5 & $6,143(7.0)$ \\
\hline $6 \geq$ & $18,925(21.5)$ \\
\hline \multicolumn{2}{|l|}{ Psychiatric conditions } \\
\hline 0 conditions & 77,030 \\
\hline 1 conditions & 8,410 \\
\hline 2 conditions & 1,891 \\
\hline 3 conditions & 410 \\
\hline 4 conditions & 75 \\
\hline 5 conditions & 15 \\
\hline$\geq 6$ conditions & 6 \\
\hline \multicolumn{2}{|l|}{ Somatic conditions } \\
\hline 0 conditions & 18,048 \\
\hline 1 conditions & 14,982 \\
\hline 2 conditions & 13,109 \\
\hline 3 conditions & 10,279 \\
\hline 4 conditions & 7,970 \\
\hline 5 conditions & 5,859 \\
\hline$\geq 6$ conditions & 17,590 \\
\hline
\end{tabular}

Note: the number of chronic conditions includes psychiatric and somatic conditions. Note that when the number of chronic conditions increases, the number of individuals decreases.

Table 2. Cross-table of prevalence and observed/expected ratio's

\begin{tabular}{lcll}
\hline & \multicolumn{2}{c}{ Observed/expected ratio } & \\
\cline { 2 - 4 } & $1.49 \leq$ & $\geq 1.50$ & Total \\
\hline Prevalence $\leq 9$ & 38 & 22 & 60 \\
$\geq 10$ & 52 & 54 & 106 \\
\hline Total & 90 & 76 & 166 \\
\hline
\end{tabular}


In total, 54 disease combinations passed the threshold for the two postulated criteria, i.e. a prevalence of $\geq 10$ in the $\mathrm{RNH}$ and with an $\mathrm{O} / \mathrm{E}$ ratio of $\geq 1.5$ (Table 4 and Appendix I).

Of these 54 combinations, $31.5 \%(n=17)$ was evaluated as 'causal relation possible', 'causal relation expected' or 'otherwise related'. Hence, $68.5 \% \quad(n=37)$ disease combinations were evaluated as 'no causal relation', i.e. according to the expert a causal relation appeared to be unlikely. By using the two selection criteria (i.e. prevalence and $\mathrm{O} / \mathrm{E}$ ratio), $51.5 \%$ of the disease combinations (17 out of 33) that were evaluated as either 'causal relation possible', 'causal relation expected' or 'otherwise related' were included for further evaluation.

Only $27.8 \%$ (37 out of 133) of the disease combinations that were evaluated as 'no causal relation' were included using the two criteria. Since all the excluded disease combinations have a low prevalence and/or a low $\mathrm{O} / \mathrm{E}$ ratio, it is unlikely that any potentially interesting disease combinations were missed. In order to identify disease combinations that are not yet known to experts (i.e. novel disease combinations), only disease combinations that were evaluated by the expert as 'no causal relation present' (i.e. an evaluation as 0) were selected for further evaluation resulting in a final list of 37 disease combinations.

Two months after the first expert evaluation, the 37 disease combinations were randomly ordered and were again valuated by a psychiatrist/scientific research in the field of psychiatry and neuropsychology. However, in order to acquire a more detailed evaluation, a medical doctor/scientific researcher in the field of psychiatry and neuropsychology and a general practitioner/ scientific researcher in the field on general practice also evaluated the disease combinations. These 37 disease combinations were evaluated using the abovementioned four categories (i.e. $0,1,2$ or 3 ).

\section{Expert Meeting}

An expert meeting was arranged in order to discuss several potential relevant disease combinations. As stated previously, the aim of this meeting was not to reach consensus among the experts, but was aimed at discussing the thoughts and ideas behind the expert's evaluation regarding the disease combinations. Five disease combinations were selected as examples of the broad scope of interactions between psychiatric and somatic conditions. The following disease combinations were discussed during the expert meeting (marked in table 3 with an asterisk ${ }^{*}$ ); appendicitis and personality disorder, uncomplicated hypertension and psychotic affective disorders, migraine disorder and personality disorder, pulmonary emphysema and depressive disorder, and lipid metabolism disorders and depressive disorder.

In the expert meeting it became apparent that the experts used different approaches when evaluating the disease combinations (see Box 1). Different risk factors, biological as well as environmental factors, were formulated as underlying causes of the disease combination at hand. Moreover, the experts had different concepts and definitions in mind when evaluating the disease combinations, which, on several occasions, explained differences in evaluation. 
Table 3. Distribution of 166 disease combinations according to prevalence, observed/expected ratio and expert evaluation

\begin{tabular}{|c|c|c|c|c|c|c|}
\hline \multirow{2}{*}{ Expert evaluation } & \multirow[t]{2}{*}{$\mathrm{N}$} & \multicolumn{4}{|c|}{ Observed/expected ratio } & \multirow[t]{2}{*}{ Total } \\
\hline & & $\leq 0.49$ & $0.5-0.99$ & $1-1.49$ & $\geq 1.5$ & \\
\hline \multicolumn{7}{|l|}{ (0) No causal relation } \\
\hline & $1-4$ & 2 & 3 & 6 & 4 & 15 \\
\hline & $5-9$ & 0 & 12 & 9 & 14 & 35 \\
\hline & $10-24$ & 1 & 7 & 16 & 12 & 36 \\
\hline & $25-49$ & 0 & 6 & 7 & 12 & 25 \\
\hline & $50-99$ & 0 & 1 & 2 & 8 & 11 \\
\hline & $100-199$ & 0 & 0 & 4 & 1 & 5 \\
\hline & $\geq 200$ & 0 & 1 & 1 & 4 & 6 \\
\hline & Total & 3 & 30 & 45 & 55 & 133 \\
\hline \multicolumn{7}{|c|}{ (1) Causal relation possible } \\
\hline & $1-4$ & 1 & 0 & 0 & 0 & 1 \\
\hline & $5-9$ & 0 & 0 & 1 & 0 & 1 \\
\hline & $10-24$ & 0 & 0 & 1 & 3 & 4 \\
\hline & $25-49$ & 0 & 0 & 0 & 1 & 1 \\
\hline & $100-199$ & 0 & 0 & 0 & 2 & 2 \\
\hline & $\geq 200$ & 0 & 0 & 0 & 1 & 1 \\
\hline & Total & 1 & 0 & 2 & 7 & 10 \\
\hline \multicolumn{7}{|c|}{ (2) Causal relation expected } \\
\hline & $1-4$ & 0 & 0 & 1 & 0 & 1 \\
\hline & $5-9$ & 0 & 0 & 0 & 1 & 1 \\
\hline & $\geq 200$ & 0 & 0 & 0 & 1 & 1 \\
\hline & Total & 0 & 0 & 1 & 2 & 3 \\
\hline \multicolumn{7}{|c|}{ (3) Related as epiphenomenon } \\
\hline & $1-4$ & 0 & 1 & 0 & 1 & 2 \\
\hline & $5-9$ & 1 & 0 & 1 & 2 & 4 \\
\hline & $10-24$ & 0 & 0 & 3 & 5 & 8 \\
\hline & $25-49$ & 0 & 0 & 1 & 1 & 2 \\
\hline & $50-99$ & 0 & 0 & 0 & 2 & 2 \\
\hline & $100-199$ & 0 & 0 & 1 & 0 & 1 \\
\hline & $\geq 200$ & 0 & 0 & 0 & 1 & 1 \\
\hline & Total & 1 & 1 & 6 & 12 & 20 \\
\hline & Total & 5 & 31 & 54 & 76 & 166 \\
\hline
\end{tabular}

Note: grey area indicates those disease combinations that were selected by means of two criteria; prevalence of $\geq 10$ in $\mathrm{RNH}$ and a $\mathrm{O} / \mathrm{E}$ ratio of $\geq 1.5$ ). Using these two criteria of interestingness, $51.6 \%$ of the disease combinations (17 out of 33) that were evaluated as either 'causal relation possible', or 'causal relation expected' or 'otherwise related' are included for further evaluation. In total, $27.8 \%$ (37 out of 133) of disease combinations that were evaluated as 'no causal relation' were selected using the two criteria. Since all the excluded disease combinations have a low prevalence and/or a low $\mathrm{O} / \mathrm{E}$ ratio, it is unlikely that any potentially interesting disease combinations are missed out on. 
Table 4. Odds Ratio(OR) and 95\% Confidence Interval (CI) for 37 (out of 54) selected psychiatric and somatic disease combinations

\begin{tabular}{|c|c|c|c|c|c|c|}
\hline \# & Somatic condition & Psychiatric condition & $\mathrm{N}$ & $\mathrm{O} / \mathrm{E}$ & OR & $95 \% \mathrm{CI}$ \\
\hline 1 & Esophageal diseases & Personality disorders & 48 & 1.97 & 2.03 & $1.52-2.72$ \\
\hline 2 & Duodenal ulcer & Suicide attempt & 15 & 1.83 & 1.86 & $1.11-3.11$ \\
\hline 3 & Duodenal ulcer & Personality disorder & 32 & 1.61 & 1.64 & $1.15-2.33$ \\
\hline 4 & Appendicitis & Schizophrenia & 14 & 1.70 & 2.37 & $1.11-5.03$ \\
\hline 5 & Appendicitis & Depressive disorder & 300 & 1.77 & 1.86 & $1.65-2.11$ \\
\hline 6 & Appendicitis & Suicide attempt & 53 & 2.37 & 2.53 & $1.90-3.36$ \\
\hline $7^{*}$ & Appendicitis & Personality disorder & 102 & 1.88 & 1.98 & $1.61-2.43$ \\
\hline 8 & Hernia, hiatal & $\begin{array}{l}\text { Affective disorders, } \\
\text { psychotic }\end{array}$ & 10 & 1.81 & 1.84 & $0.98-3.46$ \\
\hline 9 & Hernia, hiatal & Suicide attempt & 18 & 1.76 & 1.79 & $1.12-2.87$ \\
\hline 10 & Hernia, hiatal & Personality disorder & 50 & 2.01 & 2.08 & $1.56-2.78$ \\
\hline 11 & Angina pectoris & Depressive disorder & 214 & 1.68 & 1.79 & $1.56-2.05$ \\
\hline 12 & Angina pectoris & Suicide attempt & 11 & 1.99 & 2.07 & $1.48-2.89$ \\
\hline $13^{*}$ & $\begin{array}{l}\text { Uncomplicated } \\
\text { hypertension }\end{array}$ & $\begin{array}{l}\text { Affective disorders, } \\
\text { psychotic }\end{array}$ & 65 & 1.79 & 1.92 & $1.46-2.52$ \\
\hline 14 & Cerebrovascular accident & Mental retardation & 20 & 1.50 & 1.51 & $0.97-2.37$ \\
\hline 15 & Femoral fractures & Mental retardation & 20 & 3.09 & 3.21 & $2.04-5.03$ \\
\hline 16 & Dislocations & Personality disorder & 20 & 1.52 & 1.54 & $0.99-2.41$ \\
\hline 17 & Dislocations & Mental retardation & 10 & 1.73 & 1.75 & $0.94-3.29$ \\
\hline 18 & Osteoarthritis, spine & Suicide attempt & 27 & 1.53 & 1.56 & $1.06-2.30$ \\
\hline 19 & Shoulder syndrome & Suicide attempt & 32 & 1.89 & 1.95 & $1.36-2.79$ \\
\hline 20 & Osteoporosis & Suicide attempt & 29 & 1.78 & 1.83 & $1.26-1.66$ \\
\hline 21 & Meningitis/encephalitis & Personality disorder & 13 & 1.72 & 1.75 & $1.01-3.04$ \\
\hline 22 & Meningitis/encephalitis & Mental retardation & 11 & 3.31 & 3.41 & $1.87-6.24$ \\
\hline 23 & Brain concussion & Depressive disorder & 94 & 1.93 & 2.06 & $1.66-2.55$ \\
\hline 24 & Brain concussion & Suicide attempt & 20 & 3.18 & 3.31 & $2.11-5.19$ \\
\hline 25 & Brain concussion & Personality disorder & 34 & 2.23 & 2.31 & $1.63-3.26$ \\
\hline 26 & Epilepsy & Personality disorder & 48 & 2.73 & 2.87 & $2.14-3.86$ \\
\hline 27 & Epilepsy & Mental retardation & 85 & 11.01 & 13.53 & $10.69-17.14$ \\
\hline 28 & Migraine disorders & Suicide attempt & 34 & 1.88 & 1.94 & $1.37-2.75$ \\
\hline $29^{*}$ & Migraine disorders & Personality disorder & 77 & 1.75 & 1.82 & $1.44-2.30$ \\
\hline 30 & Bell palsy & Depressive disorder & 36 & 1.54 & 1.56 & $1.13-2.25$ \\
\hline 31 & $\begin{array}{l}\text { Chronic bronchitis/ } \\
\text { bronchiectasis }\end{array}$ & Personality disorder & 22 & 1.72 & 1.75 & $1.14-2.69$ \\
\hline $32^{*}$ & Pulmonary emphysema & Depressive disorder & 295 & 1.99 & 2.19 & $1.93-2.48$ \\
\hline 33 & Pulmonary emphysema & Personality disorder & 96 & 2.07 & 2.19 & $1.77-2.70$ \\
\hline 34 & Myxedema & $\begin{array}{l}\text { Affective disorders, } \\
\text { psychotic }\end{array}$ & 30 & 3.00 & 5.25 & $3.60-7.67$ \\
\hline 35 & Myxedema & Mental retardation & 19 & 1.54 & 1.56 & $0.98-2.47$ \\
\hline $36^{*}$ & Lipid metabolism disorders & Depressive disorder & 569 & 1.70 & 1.88 & $1.71-2.06$ \\
\hline 37 & Lipid metabolism disorders & Suicide attempt & 65 & 1.51 & 1.57 & $1.21-2.04$ \\
\hline
\end{tabular}

${ }^{*}$ Disease combinations selected for expert meeting. Data are reported as OR (and corresponding 95\% CI) indicating (non)independence between two chronic conditions. $\mathbb{N}$ refers to the prevalence of disease combination in $\mathrm{RNH}(\mathrm{N}=87.837)$. 
Box 1. Topics brought forward during the expert meeting

- Diseases can be related because they share common biological pathways or mechanism

- Diseases can be related because of shared psychosocial risk factors

- The diagnostic process of some diseases can cause relations between two diseases

- The medication or therapy for on disease might act as a risk factor for another disease

The multi-disciplinary expert meeting produced various reasons that could underlie the specific disease combinations; diseases could be causally related because they share similar biological or psychosocial risk factors or because one condition may act as a risk factor for the other conditions (e.g. migraine disorder and personality disorder). In addition, the experts also mentioned that a disease combination could be causally related because one condition might occur as a result of the pharmacological treatment of the other condition. For example, the experts suggested that medication for pulmonary emphysema might promote depressive symptoms, which could result in a diagnosis of a depressive disorder.

Several questions concerning diagnostic labels were brought forward during the expert meeting. For example, the question rose if general practitioners also coded a diagnosis of 'personality disorder' (i.e. ICPC code P80) when only minor personality changes, e.g. after a stroke were present (instead of a full-blown personality disorder). Moreover, the experts stated that individuals who visit their general practitioner on a regular basis have a greater likelihood of being diagnosed with a (co-morbid) medical condition than people who hardly ever visit their general practitioner. This kind of information provided insight in the thoughts and ideas regarding the relations between the diseases of the disease combinations. Even if all three experts had no idea regarding underlying relations between two diseases, when being confronted with the prevalence of the disease combination in the RNH and the corresponding $\mathrm{O} / \mathrm{E}$ ratio, the experts were surprised and intrigued which lead to additional in-depth discussion regarding the disease combination at hand.

\section{DISCUSSION}

The last decade, the amount of medical information, systematically stored in electronic primary care databases, has increased substantially. This study was aimed at discovering novel and potential relevant psychiatric and somatic disease combinations, using a broad nosological spectrum in order to utilize this enormous amount of medical data.

The present study introduces several undiscovered, potentially relevant disease combinations including appendicitis and personality disorder, uncomplicated hypertension and psychotic affective disorders, migraine disorders and personality disorder, pulmonary emphysema and depressive disorder, and lipid metabolism disorders and depressive disorders. Before these disease combinations can be investigated in-depth for plausible underlying (causal) relations, these disease combinations need to be confirmed in other, preferable general-practice based, datasets. Subsequently, cross-sectional or longitudinal studies could investigate underling shared risk factors or pathophysiological pathways.

Our results indicate that the two postulated criteria regarding prevalence and observed/expected ratio are useful to identify novel, potentially relevant patterns of multimorbidity. Disease combinations with a low prevalence and a low $\mathrm{O} / \mathrm{E}$ ratio, in 
general, are only evaluated as 'no causal relation'. Hence, by using these two criteria, hardly any disease combinations that are evaluated as 'causal relation possible' or 'causal relation expected' are excluded. The two criteria postulated in the present study can aid the medical researcher by being a first selective filter to select potentially relevant disease combinations with a plausible causal relationship.

Our expert meeting was intended to discuss the ideas and thoughts of three experts regarding plausible relationships between the diseases of several disease combinations. An expert meeting is a common way to collect opinions and knowledge from domain experts. Moreover, including experts with various, related backgrounds, will result in a broad, multi-disciplinary perspective. Our findings suggest that a multi-disciplinary expert meeting provides additional information over and above the two selection criteria and individual expert evaluations. This underscores previous research which suggest that the input of medical experts cannot be ignored in identifying undiscovered, potential relevant medical findings33.

Although the present study did not evaluate all possible ICPC codes that describe chronic conditions, the present study does include a large spectrum of frequently occurring psychiatric and somatic conditions in order to identify novel, potentially relevant disease combinations. Previous research used only a limited number of chronic conditions to identify frequently occurring disease combinations. For example, a study by Laux and colleagues investigated disease combinations in only five chronic conditions ${ }^{34}$. Other studies focused on combinations of systems, such as respiratory and cardiac systems, rather that on single diseases ${ }^{34}$. Secondly, the large sample size increases the generalisability of our results. Thirdly, the majority of individuals registered by means of the $\mathrm{RNH}$ are between 40 and 59 years of age. Hence, the present study was not only directed at older people (i.e. aged 80 and over).

Our findings must be interpreted in light of some possible limitations. First, some caution is needed when appraising disease combinations that just pass the selection criteria, i.e. prevalence of $\geq 10$ and an $\mathrm{O} / \mathrm{E}$ ratio of $\geq 1.5$. Hence, in order to select those disease combinations of which the two diseases are most likely to be causally related, the threshold for these two criteria needs to be augmented (e.g. disease combinations with a O/E ratio of $2.5 \geq$ ). For example, in case of finding new biological and psychosocial causal pathways it might be preferable to explore the subset of more prevalent disease combinations with higher odds ratio's. Secondly, the diagnoses that are registered in the RNH database reflect the GPs perspective of the health status and relevant health problems of the patient. As a result, some health problems may be missing because a patient did not report them to the GP or because the GP does not judge them to be clinically significant ${ }^{1}$. Nevertheless, the number of missing health problems appears to be small, especially in the case of permanent, chronic conditions that require medical care ${ }^{25}$.

Despite these drawbacks, this study used a large nosological spectrum to identify novel and potential relevant occurring disease combinations by means of a general practice based population. Our findings demonstrate that using two selection criteria in combination with an expert evaluation phase is useful in the practice of identifying novel, relevant disease combinations. Increasing our understanding regarding co-occurring diseases can improve 
disease diagnosis and management for individuals with multimorbidity. The present results need to be confirmed in other, preferable general practice based datasets. In addition, the presence of the potential relevant disease combinations introduced in the present study should be investigated in specific subgroups, i.e. based on age or sex. As a result, health care delivery can be adjusted to those subgroups which are more prone to experience certain disease combinations.

\section{CONCLUSIONS}

The present study demonstrates that a knowledge discovery approach can be used to analyze large medical databases in order to identify disease combinations not (yet) known to medical experts. As is inherent to exploratory and hypotheses generating research, other studies are needed to replicate the present findings. Subsequently, cross-sectional and longitudinal studies are needed to answer whether or not the disease combinations presented here are indeed (causally) related.

The exploratory method employed in the present study, in which two selection criteria were combined with an expert evaluation phase, provides novel avenues to identify novel and relevant disease combinations. In addition, our findings indicate that general practice based databases are an excellent resource for exploratory research. Careful and precise registration of medical conditions is an important prerequisite hereof. 


\section{REFERENCES}

1. M. van den Akker, F. Buntinx, J.F. Metsemakers, S. Roos, and J.A. Knottnerus. Multimorbidity in general practice: prevalence, incidence, and determinants of co-occurring chronic and recurrent diseases. J. Clin. Epidemiol., 1998;51(5):367-375.

2. J. Neeleman, J. Ormel, and R.V. Bijl. The distribution of psychiatric and somatic III health: associations with personality and socioeconomic status. Psychosom. Med., 2001;63(2):239-247.

3. M. Hotopf, R. Mayou, M. Wadsworth, and S. Wessely. Temporal relationships between physical symptoms and psychiatric disorder. Results from a national birth cohort. Br. J. Psychiatry, 1998; 173:255-261.

4. J.S. Gonzalez, S.A. Safren, E. Cagliero, D.J. Wexler, L. Delahanty, E. Wittenberg, M.A. Blais, J.B. Meigs, and R.W. Grant. Depression, self-care, and medication adherence in type 2 diabetes: relationships across the full range of symptom severity. Diabetes Care, 2007;30(9):2222-2227.

5. L.G. Glynn, J.M. Valderas, P. Healy, E. Burke, J. Newell, P. Gillespie, and A.W. Murphy. The prevalence of multimorbidity in primary care and its effect on health care utilization and cost. Fam. Pract., 2010.

6. C.M. Boyd and M. Fortin. Future of multimorbidity research: How should understanding of multimorbidity inform health system design? Public Health Rev., 2010;32(2):451-474.

7. S. Aarts, M. van den Akker, M.P. van Boxtel, J. Jolles, B. Winkens, and J.F. Metsemakers. Diabetes mellitus type II as a risk factor for depression: a lower than expected risk in a general practice setting. Eur. J. Epidemiol., 2009;24(10):641-648.

8. M. de Hert, V. Schreurs, D. Vancampfort, and V.A.N.W. R. Metabolic syndrome in people with schizophrenia: a review. World Psychiatry, 2009;8(1):15-22.

9. H. van den Bussche, D. Koller, T. Kolonko, H. Hansen, K. Wegscheider, G. Glaeske, E.C. von Leitner, I. Schafer, and G. Schon. Which chronic diseases and disease combinations are specific to multimorbidity in the elderly? Results of a claims data based cross-sectional study in Germany. BMC Public Health, 2011;11:101.

10. A. Wong, H.C. Boshuizen, F.G. Schellevis, G.J. Kommer, and J.J. Polder. Longitudinal administrative data can be used to examine multimorbidity, provided false discoveries are controlled for. J. Clin. Epidemiol., 2011;64(10):1109-1117.

11. A. Marengoni, D. Rizzuto, H.X. Wang, B. Winblad, and L. Fratiglioni. Patterns of chronic multimorbidity in the elderly population. J. Am. Geriatr. Soc., 2009;57(2):225-230.

12. J.N. Struijs, C.A. Baan, F.G. Schellevis, G.P. Westert, and G.A. van den Bos. Comorbidity in patients with diabetes mellitus: impact on medical health care utilization. BMC Health Serv. Res., 2006;6:84.

13. H. Karsten and H. Suominen. Mining of clinical and biomedical text and data: editorial of the special issue. Int. J. Med. Inf., 2009;78(12):786-787.

14. C. Piatetsky-Shapiro. Knowledge Discovery in Real Databases: A Report on the IJCAI-89 Workshop. Artificial Intelligence Magazine, 1991;11(5):68-70.

15. G. Piatetsky-Shapiro. Data mining and knowledge discovery 1996 to 2005: overcoming the hype and moving from "university" to "business" and "analytics". Data Mining and Knowledge Discovery, 2007;15(1):99-105.

16. A. Silberschatz and A. Tuzhilin. What Makes Patterns Interesting in Knowledge Discovery Systems. IEEE Transactions on Knowledge and Data Engineering, 1996;8(6):970-974.

17. M. Roos, M.S. Marshall, A.P. Gibson, M. Schuemie, E. Meij, S. Katrenko, W.R. van Hage, K. Krommydas, and P.W. Adriaans. Structuring and extracting knowledge for the support of hypothesis generation in molecular biology. BMC Bioinformatics, 2009;10 S9.

18. N. Lavrac, M. Bohanec, A. Pur, B. Cestnik, M. Debeljak, and A. Kobler. Data mining and visualization for decision support and modeling of public health-care resources. Journal of Bio-medical Informatics, 2007;40(4):438-447. 
19. A. Oztekin, D. Delen, and Z.J. Kong. Predicting the graft survival for heart-lung transplantation patients: an integrated data mining methodology. Int. J. Med. Inf., 2009;78(12):84-96.

20. J.F.M. Metsemakers, P. Hoppener, J.A. Knottnerus, R.J. Kocken, and C.B. Limonard. Computerized health information in The Netherlands: a registration network of family practices. Br. J. Gen. Pract., 1992;42(356):102-106.

21. C.c.o. WONCA. ICPC International Classification of Primary Care. 1987, Oxford: Oxford University Press.

22. J.F. Metsemakers, J.A. Knottnerus, G.J. van Schendel, R.J. Kocken, and C.B. Limonard. Unlocking patients' records in general practice for research, medical education and quality assurance: the Registration Network Family Practices. Int. J. Biomed. Comput., 1996;42(1-2):43-50.

23. J.A. Knottnerus. Role of the electronic patient record in the development of general practice in The Netherlands. Methods Inf. Med., 1999;38(4-5):350-354.

24. M. Van den Akker, G.H.L.M. Franssen, F. Buntinx, J.F.M. Metsemakers, and J.A. Knottnerus. The reliability of register-based patient characteristics. Archives of Public Health, 1997;55:231-238.

25. J.F. Metsemakers, J.A. Knottnerus, G.J. van Schendel, R.J. Kocken, and C.B. Limonard. Unlocking patients' records in general practice for research, medical education and quality assurance: the Registration Network Family Practices. Int. J. Biomed. Comput., 1996;42(1-2):43-50.

26. D. Howell. Statistical Methods for Psychology. 2002, California: Wadsworth Publishing Company

27. I.H. Witten and E. Frank. Data mining: practical machine learning tools and techniques. 2nd. ed. 2005, San Francisco: Elsevier.

28. L. Batstra, E.H. Bos, and J. Neeleman. Quantifying psychiatric comorbidity--lessions from chronic disease epidemiology. Soc. Psychiatry Psychiatr. Epidemiol., 2002;37(3):105-111.

29. G.L. Grunkemeier and Y. Wu. What are the odds? Ann. Thorac. Surg., 2007;83(4):1240-1244.

30. J.M. Bland and D.G. Altman. Statistics notes. The odds ratio. BMJ, 2000;320(7247):1468.

31. T.J. Bouchard, Jr. and M. Hare. Size, performance, and potential in brainstorming groups. J. Appl. Psychol., 1970;54(1):51-55.

32. T.J. Bouchard, Jr. Training, motivation, and personality as determinants of the effectiveness of brainstorming groups and individuals. J. Appl. Psychol., 1972;56(4):324-331.

33. A. Oztekin, D. Delen, and Z.J. Kong. Predicting the graft survival for heart-lung transplantation patients: an integrated data mining methodology. Int. J. Med. Inf., 2009;78(12):84-96.

34. G. Laux, T. Kuehlein, T. Rosemann, and J. Szecsenyi. Co- and multimorbidity patterns in primary care based on episodes of care: results from the German CONTENT project. BMC Health Serv. Res., $2008 ; 8: 14$. 


\section{APPENDIX I}

Odds Ratio (OR) and 95\% Confidence Intervals (CI) for the remaining 17 (out of 54) psychiatric and somatic disease combinations

\begin{tabular}{|c|c|c|c|c|c|c|}
\hline \# & Somatic condition & Psychiatric condition & $\mathrm{N}^{\mathrm{a}}$ & $\mathrm{O} / \mathrm{E}$ & $\mathrm{OR}^{\mathrm{b}}$ & $95 \% \mathrm{CI}$ \\
\hline 1 & Esophageal diseases & Suicide attempt & 17 & 1.69 & 1.72 & $1.06-2.79$ \\
\hline 2 & Diverticulosis & Affective disorders, psychotic & 10 & 1.63 & 1.63 & $0.87-3.07$ \\
\hline 3 & $\begin{array}{l}\text { Cardiovascular } \\
\text { abnormalities }\end{array}$ & Mental retardation & 18 & 5.44 & 5.76 & $3.57-9.30$ \\
\hline 4 & $\begin{array}{l}\text { Cerebrovascular } \\
\text { accident }\end{array}$ & Depressive disorder & 208 & 2.13 & 2.34 & $2.02-2.71$ \\
\hline 5 & $\begin{array}{l}\text { Cerebrovascular } \\
\text { accident }\end{array}$ & Suicide attempt & 25 & 1.99 & 2.04 & $1.36-3.06$ \\
\hline 6 & Epilepsy & Affective disorders, psychotic & 11 & 2.81 & 2.90 & $1.58-5.30$ \\
\hline 7 & Epilepsy & Suicide attempt & 16 & 2.21 & 2.26 & $1.37-3.73$ \\
\hline 8 & Cluster Headache & Depressive disorder & 14 & 2.35 & 2.55 & $1.45-4.47$ \\
\hline 9 & $\begin{array}{l}\text { Tuberculosis, } \\
\text { Pulmonary }\end{array}$ & Depressive disorder & 45 & 1.87 & 1.99 & $1.45-2.70$ \\
\hline 10 & $\begin{array}{l}\text { Malignant neoplasm } \\
\text { bronchus/lung }\end{array}$ & Depressive disorder & 16 & 1.73 & 1.80 & $1.08-3.02$ \\
\hline 11 & $\begin{array}{l}\text { Chronic bronchitis/ } \\
\text { bronchiectasis }\end{array}$ & Depressive disorder & 68 & 1.67 & 1.74 & $1.36-2.24$ \\
\hline 12 & Hyperventilation & Depressive disorder & 222 & 2.80 & 3.22 & $2.79-3.73$ \\
\hline 13 & $\begin{array}{l}\text { Benign neoplasm of } \\
\text { thyroid gland }\end{array}$ & Depressive disorder & 13 & 2.24 & 2.42 & $1.35-4.32$ \\
\hline 14 & Goiter & Depressive disorder & 98 & 2.30 & 2.51 & $2.03-3.11$ \\
\hline 15 & $\begin{array}{l}\text { Hyperthyroidism/ } \\
\text { thyrotoxicosis }\end{array}$ & Depressive disorder & 133 & 2.08 & 2.26 & $1.88-2.71$ \\
\hline 16 & Myxedema & Depressive disorder & 186 & 2.07 & 2.25 & $1.93-2.63$ \\
\hline 17 & Diabetes Mellitus & Depressive disorder & 487 & 1.56 & 1.68 & $1.52-1.85$ \\
\hline
\end{tabular}

Data are reported as OR (and corresponding 95\% CI) indicating (non)independence between two chronic conditions. $\mathrm{N}$ refers to the prevalence of disease combination in $\mathrm{RNH}(\mathrm{N}=87.837)$. 
CHAPTER 9

General discussion 
General practitioners are increasingly confronted with patients who suffer from multimorbidity, the co-occurrence of two or more chronic medical conditions. Multimorbidity is associated with poor quality of life $e^{1-3}$, physical disability ${ }^{4,5}$, higher use of health care facilities ${ }^{6}$ and higher mortality risk $^{7}$. In general, individuals who suffer from multimorbidity require more complex health care compared to individuals with a single medical condition ${ }^{6}$. For example, individuals with multimorbidity consult more health care professionals than individuals with single morbidity. Managing multimorbidity by health care providers is therefore complicated, not in the least because of possible interactions between the occurring medical conditions ${ }^{8}$.

In order to improve health care delivery for and quality of life in patients with multimorbidity, it is important to provide a more detailed picture of multimorbidity. For this purpose, the following two research questions were postulated: 1) to report on several adverse health effects and related concepts associated with multimorbidity, and 2) to introduce and apply innovative research strategies useful for analyzing large amounts medical data. The current chapter summarizes the main findings and relates them to the current medical literature. Possible limitations, with special attention to methodological considerations, are also discussed. In addition, implications for daily clinical practice regarding multimorbidity are put forward. Recommendations for future research on multimorbidity are presented at the end of this chapter.

\section{MAIN FINDINGS}

The studies included in this thesis underscore that multimorbidity is highly prevalent in the adult population. Prevalences of more than $50 \%$ of the population under study are reported ${ }^{9,10}$. Conversely, a much smaller percentage is without any chronic conditions. Although the prevalence of multimorbidity varies considerably in the medical literature, our prevalence estimates of multimorbidity are in congruence with those reported in a recently published systematic review ${ }^{11}$.

\section{Adverse Health Consequences and Related Constructs}

\section{Multimorbidity and Cognitive Functioning}

Various factors related to functional health have been proposed as prognostic factors for pathological cognitive functioning ${ }^{12}$. In this notion, the relation between morbidity and multimorbidity on one side and subjective and objective cognitive functioning on the other was studied. The following questions were postulated: 1) Is multimorbidity associated with memory complaints? and 2) What is the longitudinal association between multimorbidity and cognitive functioning?

The findings related to the first research question illustrate that individuals with one or more chronic medical conditions reported more subjective memory complaints than their healthy counterparts. This association was more profound in individuals with multimorbidity. Although this study provides evidence for a strong association between multimorbidity and cognitive complaints, the question remains whether this observed association reflects an objective decrease in cognitive functioning. Another research 
question was aimed at investigating the relation between multimorbidity and objective cognitive performance. The results from this longitudinal study with a 12 year follow-up, underscore earlier claims that morbidity can be considered as a possible mediating factor in cognitive dysfunction ${ }^{12}$. Nevertheless, the effects of suffering from a single chronic condition and multimorbidity on cognition appear to be small in a normal healthy aging population.

It might be argued that the presence of diseases makes an individual more prone to experience cognitive problems since the suffering from one or more chronic conditions might occupy the cognitive resources of an individual (e.g. through 'worrying') ${ }^{13}$. Moreover, the cognitive problems (older) people experience may easily be interpreted as the first signs of cognitive decline instead of normal forgetfulness that increases with age. Special attention, aimed at improving one's beliefs and expectations regarding their cognitive functioning, could be given to these patients.

\section{Multimorbidity and Functional Health}

To gain more insight in the association between multimorbidity and adverse health effects, we investigated if the contribution of multimorbidity to physical and mental health is temporary or long lasting. This study claims that the decrease in self-perceived physical functioning caused by multimorbidity may be permanent and may even increase over time. Moreover, the association between single morbidity and multimorbidity on one side and mental functioning on the other seems to disappear over time. Our findings suggest that multimorbidity is associated with long-term physical problems rather than with mental problems. Since physical problems are known to underlie several adverse outcomes, including hospitalization and nursing home admission ${ }^{14}$, early disease diagnosis and appropriate disease management that is aimed at ameliorating or postponing these physical problems is therefore an important aim.

\section{Multimorbidity and Related Constructs}

The term multimorbidity has been, and often still is, used interchangeable with other gerontological constructs such as frailty and disability. The studies presented in this thesis indicate that individuals who were frail but who did not suffer from multimorbidity and/or disability did not show an increased risk for nursing home admission or mortality. In addition, while mortality seems to be primarily driven by disease burden, functional limitations seem to be the driving force behind nursing home admission. It might therefore be concluded that the adverse health risks associated with frailty in the general older adult population may primarily be driven by multimorbidity and disability.

\section{Diabetes mellitus and Depression}

Cross-sectional studies have demonstrated a high presence of depression in individuals with diabetes mellitus and depression, a specific pattern of multimorbidity. Although our results claim that patients with type II diabetes were more likely to develop subsequent depression than persons without a history of type II diabetes, the association between diabetes and depression obtained in our longitudinal study was weaker than previously reported. Type II 
diabetes might lead to depression because the rigors of managing diabetes can be stressful or because of the biochemical changes caused by diabetes mellitus or its treatment ${ }^{15}, 16$. Although several explanations for the association between type II diabetes and depression have been postulated, the pathophysiological mechanisms underlying this particular disease combination remains inconclusive. Since this disease combination is known for its substantial disease burden ${ }^{17}$, better recognition of depression in patients with type II diabetes could the adverse effects of this pattern of multimorbidity.

\section{Innovative Analyzing Strategies}

In the last decade, large volumes of medical data are collected in hospitals, clinics and general practices because of a switch to computerized medical record keeping ${ }^{18}$. Since traditional analyzing techniques are less adequate when the amount and complexity of data increases, there is a growing need for practical applications to assist medical researchers in extracting (new) information and knowledge from large amounts of medical data.

Knowledge discovery in Databases (KDD) describes the process of (semi)automatically searching large volumes of data for novel and potentially relevant patterns or associations ${ }^{19}$, ${ }^{20}$. In contrast to traditional analyzing methods which are characterized as 'confirmatory' in nature, knowledge discovery techniques serve an exploratory process, aimed at discovering unexpected patterns and associations, without a priori postulation of a priori hypotheses ${ }^{21}$. Consequently, KDD causes no or limited conformation biases. Moreover, KDD extends traditional analysis techniques by taking advantage of new technologic developments, such as the increase in computational power ${ }^{22}$.

As is the case with all analyzing methods, uncritical appraisal of implementation of KDD applications (sometimes referred to as 'data dredging' or 'fishing expeditions') can lead to the discovery of erroneously, meaningless or invalid patterns ${ }^{23}$. Understanding and applying KDD requires a different research perspective compared to using traditional statistical analyzing approaches; potentially interesting findings found should be interpreted solely as suggestions for subsequent, in-depth, research. Moreover, the interpretation of the medical findings obtained by means of KDD approaches requires careful evaluation from medical domain experts. Since the amount of data stored in medical database is only expected to rise, new analysing strategies are required to give meaning to these large and complex medical datasets. By using exploratory analyzing approaches, medical researchers are able to exploit large amounts of medical data in order to gain new insights and knowledge.

Patterns of multimorbidity

Despite the high prevalence of multimorbidity, knowledge concerning frequently occurring disease combinations is still scarce. This might be due to the fact that we face an enormous amount of possible disease combinations when investigating possible combinations of all co-occurring chronic medical conditions. For patients as well as for health care professionals, multimorbidity becomes particularly challenging when one condition influences the care of the other conditions through, for example, interactions between therapies and contraindications to medication. Consequently, knowledge of frequently co-occurring diseases may improve disease diagnosis in individuals with or at risk 
for multimorbidity which, as a result, can improve health care outcomes in patients with multimorbidity.

In this thesis, several novel, potentially relevant patterns of psychiatric and somatic conditions were introduced including appendicitis and personality disorder, depressive disorder and pulmonary emphysema, migraine disorder and lipid metabolism disorder, and uncomplicated hypertension and psychotic affective disorders. These disease combinations need to be confirmed in other general practice-base databases. Subsequently, prospective cross-sectional and longitudinal studies should evaluate underling shared risk factors or pathophysiological pathways in order to assess plausible causal relationships among the medical conditions within these disease combinations. Identification of these novel and potentially relevant disease combinations in a general practice database ${ }^{24}$, promotes hypothesis-driven studies regarding patterns of multimorbidity.

\section{METHODOLOGICAL CONSIDERATIONS}

\section{Definition and Operationalization of Multimorbidity}

A major problem in research on multimorbidity is the lack of a universally accepted definition and operationalization of multimorbidity ${ }^{25-27}$, which complicates the comparability and generalisability of studies on multiple pathology. The studies presented in this thesis made use of the widely used conceptualization postulated by van den Akker and colleagues who defined multimorbidity by 'the co-occurrence of multiple chronic or acute diseases and medical conditions within one person' 28 .

In this thesis, multimorbidity was assessed by means of a simple disease count. Although a simple disease count does not takes disease severity into account, simply counting the number of disease an individual is affected by is a simple and efficient approach. This assessment of multimorbidity assumes that every additional disease has a similar additive effect on the outcome at hand. A simple disease count is particularly useful when considering a large nosological spectrum to study multimorbidity. Using a large nosological spectrum is a prerequisite for research on multimorbidity since the exclusion of large number of diseases could yield a non-realistic picture of multimorbidity. Moreover, the set of included diseases is not only decisive for the prevalence of multimorbidity but also highly affects the number of conditions present in an individual. When evaluating a large nosological spectrum, making use of a simple disease count is often preferred over other approaches to operationalized multimorbidity. For example, the use of specific disease clusters or combinations of diseases are not feasible when using a large nosological spectrum, especially in terms of labour intensiveness and lack of statistical power because of low prevalences.

By using a simple disease count to assess multimorbidity, individuals are often divided into three, mutually exclusive, groups: healthy individuals (i.e. the reference group), individuals with single morbidity (i.e. one disease present) and individuals with multimorbidity (i.e. $\geq 2$ diseases present). In this thesis, the latter two groups were compared to the reference group in order to study the effects of a single disease or multimorbidity on a specific outcome measure (e.g. cognition and functional health). The 
question is if dividing individuals into these three mutually exclusive groups is the most optimal way to discriminate between groups of individuals. Hence, it remains to be investigated which groups can best be formed when studying multimorbidity.

\section{Characteristics of the Databases}

In order to provide a concise and unambiguous picture of multimorbidity, the present thesis presents findings obtained by means of national and international databases as well as general practice based data and data on community-dwelling individuals. Each of the three databases has its own rationale, objectives and design. Consequently, every database contains different characteristics which will be outlined hereafter.

\section{The Registration Network Family Practices}

To advance our knowledge on multimorbidity, structural, objective information regarding chronic medical conditions is highly imperative. Therefore, four of the eight studies included in this thesis were carried out within the context of the Registration Network Family Practices (RegistratieNet Huisartspraktijken, RNH). The RNH is a continuously updated database, which contains the medical records of patients from 21 family practices, supported by 65 general practitioners $(\mathrm{GPs})^{29,30}$. In the $\mathrm{RNH}$, health problems are only coded by the GP when they are permanent (no recovery expected), chronic (duration longer than 6 months) or recurrent (more than three recurrences within 6 months), or when they have lasting consequences for the functional status or prognosis of a patient.

The studies in this thesis which were conducted by means of RNH focused on major health problems. Hence, all ICPC codes representing symptoms or complaints as well as pregnancy and delivery, test results not leading to a diagnosis, variations of normal functioning, superficial injuries and risk factors were excluded. Nevertheless, it remains possible that a medical condition that was coded by the GP, at one point becomes 'inactive'; for example, a GP could label 'depression' from 'active' to 'inactive' when being confronted with an individual, who did suffer from depression (i.e. an active ICPC code), but, at the given time, has no depressive symptoms (i.e. an inactive ICPC code). However, since health problems in the RNH are only coded when they are permanent, chronic or recurrent, or when they have lasting consequences for the functional status or prognosis of the patient, the studies in this thesis used the lifetime prevalence of medical conditions.

In the Netherlands, the general practitioner delivers continuous care and acts as a gatekeeper to other health care facilities. Nevertheless, studies based on primary care data can result in an underestimation of the prevalence of single morbidity and multimorbidity, since the GP can only register those medical conditions that are brought to his or her attention. However, non-attendees' in general practice showed little serious and chronic pathology ${ }^{31}$. It might be argued that the clinical burden of multimorbidity is higher in general practice than would be expected from data collected for the general population. This highlights the importance of having prevalence estimates at the practice level, and the development and implementation of practice-based epidemiological research.

The quality of the data is ascertained by ample instruction and training sessions, regular regional consensus groups, quality control audits, an online thesaurus available 
during data-entry and systematic control for erroneous or missing entries ${ }^{29}$. In conclusion, the studies presented in this thesis advocate that the RNH can be seen as a precise sample frame that provides a objective, general practice based view on multimorbidity.

\section{The Maastricht Aging Study}

The Maastricht Aging Study (MAAS) is a longitudinal cohort study on the determinants of successful aging 32,33 . After the baseline assessment in 1992-1993, all participants were reassessed after a 3, 6, 9 and 12 years. In total, 1823 randomly selected individual (aged 24 to 81 years), participated at the baseline of the MAAS. RNH morbidity data were linked with questionnaire and test data of MAAS. In contrast to cross-sectional research which may at best suggest correlation, a longitudinal design like MAAS permits conclusions regarding the long-term effects of multimorbidity.

Because of the large sample frame and large age range of the participants, findings from studies carried out within MAAS are generalizable to the general population. However, relatively 'unhealthy' individuals might be underrepresented in MAAS because of, for example, sample bias, admission to nursing homes or premature mortality. This 'healthy survivor effect' may have lead to an underestimation of the associations found in some of the studies present in this thesis. Repeating these studies in more medically disadvantaged populations, such as those in nursing homes or hospitals, might lead to more profound associations between multimorbidity and the adverse health effect at hand.

\section{Age, Gene/Environmental Susceptibility Study}

The Age, Gene/Environmental Susceptibility (AGES Reykjavik) Study ${ }^{34}$, which consisted of more than 5,000 individuals (75+ years) living in Iceland, was used to investigate the relation between multimorbidity, frailty and disability. Findings from this large study sample can be generalized to geriatric populations in other Western countries. Some caution is warranted when generalizing these findings to younger individuals or to older individuals in more restricted populations, such as those in nursing homes and hospitals wince these populations are expected to differ from the geriatric populations as a whole.

\section{Public Health Service}

The study based on data from the Public Health Service (Geneeskundige Gezondheidsdienst; GGD) consisted of more than 15,000 community-dwelling individuals (55+ years) living in the most southern part of the Netherlands, and may therefore not only be generalized to the remaining Dutch population (i.e. within the same age range), but may also be translated to comparable populations in other Western countries.

\section{CONCLUSIONS}

This thesis contributes to the growing collection of knowledge regarding multimorbidity by reporting on several adverse health effects, related constructs, innovative analyzing strategies and co-occurring psychiatric and somatic disease combinations. 
The empirical studies in this thesis underscore that general practitioners are increasingly being confronted with individuals with multimorbidity ${ }^{35}$. Individuals with multimorbidity pose special challenges for general practitioners, not in the least because one condition might influence the care of other conditions (e.g. interaction between treatments) ${ }^{8}$.

Our results suggest that the relation between multimorbidity and physical functioning is more profound than the relation between multimorbidity and other adverse health effects such as cognitive and mental functioning. It could therefore be argued that general practitioners should especially focus on the long-lasting reduction in physical functioning that accompanies multimorbidity. Since physical problems are known to underlie several adverse outcomes, appropriate disease management for and disease monitoring of individuals with multimorbidity seems to be of utmost importance. By providing this kind of health care, general practitioners can alleviate, ameliorate or even prevent physical detoriation in patients with multimorbidity.

Individuals with one or more co-occurring psychiatric conditions are known to experience more, and more severe, adverse health effects than individuals with merely somatic multimorbidity ${ }^{36}$. For example, the presence of comorbid psychiatric condition is related to other health effects including non-adherence of self-care27 (e.g. improper use of medication) and prolonged recovery time. General practitioners should be aware of the possible presence of psychological symptoms, which can further complicate disease management in these complex patients ${ }^{37}$. Hence, these patients require special care and disease monitoring to improve their mental health problems, which, in turn, might improve outcomes related to their somatic conditions. Gaining knowledge regarding psychiatric and somatic disease combinations can contribute to improvement in health care for individuals with these kinds of multimorbidity patterns.

Our results suggest that frailty, as a distinct concept, has little discriminant validity over and above the presence of multimorbidity and disability when predicting nursing home admission and mortality. Hence, information regarding the presence of co-occurring diseases and disability (as assessed using five short questions) seems to be sufficient in order to identify those patients that are at increased risk for adverse outcomes. Consequently, it seems redundant for general practitioners to assess frailty characteristics in order to classify the most vulnerable (older) individuals.

The results also demonstrates that knowledge discovery approaches can explore and model large amounts of medical data and documents to extract previously unknown, hidden and potentially relevant medical information. A knowledge discovery approach was employed to identify disease combinations not yet known to medical experts by means of a large general practice based dataset. Several novel and potentially relevant diseases combinations, which serve as an example of the broad range of possible disease interactions, are introduced. As is the case with all exploratory and hypotheses generating research, our findings need to be replicated in other, preferably general practice based, datasets. Subsequently, hypothesis confirming studies are needed to investigate the identified disease combinations pattern in more detail (e.g. underlying pathogenesis). In addition, our findings suggest that general practice based data are an excellent resource for exploratory research. Careful and precise registration of (chronic) medical conditions is an important 
prerequisite hereof. Although KDD is not a universal solution, it can provide novel techniques in order to gain knowledge on multimorbidity or other medical topics. Because of its ability to effectively and efficient analyse large amounts of data, knowledge discovery approaches are expected to continue to gain popularity in medical research.

\section{IMPLICATIONS}

Individuals with multimorbidity often require complex health care with special attention, knowledge and skills from multiple health care providers, often located in different health care settings ${ }^{6}$. These health care providers are often focused on only one medical condition and may therefore fail to notice any detoriation or problems in other health-related aspects $^{38}$. For many individuals with multimorbidity, this fragmentation of care may lead to various health problems (e.g. adverse drug effects, hospitalization), not in the least because of the complex medication management and treatment regimes which are often contradictory. Consequently, health care for individuals with multimorbidity should not be focused on single medical conditions but should instead adopt a more holistic approach. Findings of this thesis underscore the need to move away from a more disease-specific approach towards a more integral based approach of the individual with multimorbidity ${ }^{9,35}$. To illustrate, the present thesis designates that general practitioners should not only be aware of the physical problems in individuals with multimorbidity but should also pay attention to the cognitive status of these individuals.

The lack of clinical practice guidelines (CPGs) for individuals with multimorbidity complicates a person-centred, multidimensional health care approach. Although CPGs provide detailed information for managing single diseases, they fail to address how to optimally integrate these guidelines within the practice of health care for multimorbidity 32. However, the consideration of people with multimorbidity is essential considering its increasing prevalence. In general, recommended guidelines intended for single medical conditions might be impractical, irrelevant or even harmful for individuals several cooccurring medical conditons ${ }^{38}$. For example, an individual with multimorbidity might be treated with multiple medications, which increases the risk of adverse drug events. Moreover, multimorbidity is also associated with potentially avoidable hospitalization ${ }^{39}$. Simply translating the standard clinical practice guidelines to individuals with multiple medical conditions may not be suitable for the complex medical situations of individuals with multimorbidity and might thereby diminish the quality of health care for these patients ${ }^{38}$. Since adding a large combination of possible comorbid medical conditions to current, standard CPGs is cumbersome, a critical first step might be to expand the standard CPGs by incorporating adapted guidelines for frequently occurring co-morbidities (e.g. a depressive disorder in patients diagnosed with diabetes mellitus type II or in patients diagnosed with Chronic Obstructive Pulmonary Disease, COPD). By doing so, at least for frequently occurring disease combinations, optimal care can be provided.

\section{Electronic Patient Records for Medical Research}

The present thesis underscores that electronic medical records (EMRs) are an excellent resource for scientific research ${ }^{40}$ since they provide comprehensive, standardized and 
objective medical information (e.g. by using EMRs the presence of medical conditions or the combinations thereof can be identified which may render self-reported medical status redundant). In order to use EMRs for scientific purpose, several requisites are needed. For example, ample instruction, training sessions and regular consensus groups are a necessity in order to inform those health care professionals that provide electronic patient information. Moreover, a standard coding system needs to be used to increase the objectiveness of the information (e.g. International Classification of Primary Care; $\left.\mathrm{ICPC}^{41}\right)$. In addition, a systematic control program, that can handle erroneous or missing entries, is necessary to maintain a good quality of the data at hand ${ }^{29}$. Besides, coding sociodemographic patient characteristics and relevant past and current health problems, EMRs may also include more general patient information such as recent and past appointments with health care providers, and medication use. This kind of patient related information might render EMRs even more useful for scientific research. In conclusion, EMRs can provide a rich data source with the potential to answer key research questions that can direct or indirectly improve health care.

\section{RECOMMENDATIONS FOR FUTURE RESEARCH}

The studies presented in this thesis point out several important topics that should be addressed in future research in the field of multimorbidity. Firstly, there is an increasing need for a universally accepted definition and operationalization of multimorbidity that differentiates this term from comorbidity since using these two terms interchangeable may lead to indistinctness and incomparable results regarding multimorbidity. Therefore, Van den Akker and colleagues proposed a distinction between comorbidity and multimorbidity ${ }^{42}$. A general acceptance of the difference between multimorbidity and comorbidity may prevent ambiguity of both concepts. We therefore propose the definition by van den Akker and colleagues, who defines multimorbidity as 'the co-occurrence of multiple chronic or acute diseases and medical conditions within one person' ${ }^{\prime 2}$. Consensus on the operationalization of multimorbidity is hard to accomplish since the assessment of multimorbidity highly depends on the availability and quality of the data and, of course, on available financial resources at hand. Therefore, a single operationalization of multimorbidity that would be applicable in all circumstances seems unfeasible. Consequently, medical researchers should thoroughly report how they handled the methodological problems that arise when studying multimorbidity. This will shed light on the comparability and generalisability of these studies.

Secondly, evaluating patterns of multimorbidity might be particularly important for future research. Medical conditions might co-occur within an individual if the conditions are caused or precipitated by one another directly. Even when two or more conditions do not affect each other directly, they might still share the same risk factors or precursors ${ }^{43}$. Knowledge on frequently occurring disease combinations may therefore facilitate and accelerate disease diagnosis. The extent to which frequently occurring disease combinations represent general disease susceptibility also deserves future exploration.

Thirdly, in order to enable medical researchers to properly make use exploratory, hypothesis-driven techniques more information regarding $\mathrm{KDD}$ is needed, e.g. best 
practices of KDD, which suit different medical problems, should be outlined in more detail. Since usage and interpretation of the results of these methods requires significant expertise, there is a strong need for more collaboration between KDD experts and medical experts.

Fourthly, because of the high prevalence of multimorbidity, targeted interventions programs especially developed for patients with multiple conditions should be given priority. Targeted intervention programs are an important prerequisite for reducing the prevalence of multimorbidity and for diminishing its adverse consequences. Unfortunately, to date, the factors on which targeted intervention programs should be directed in order to improve health outcomes for individuals with multimorbidity remain unclear. To accomplish this, future research efforts should focus on the risk factors for multimorbidity. 


\section{REFERENCES}

1. E. Loza, J.A. Jover, L. Rodriguez, and L. Carmona. Multimorbidity: Prevalence, Effect on Quality of Life and Daily Functioning, and Variation of This Effect When one Condition Is a Rheumatic Disease. Semin. Arthritis Rheum., 2008;38(4):312-319.

2. M. Fortin, M.F. Dubois, C. Hudon, H. Soubhi, and J. Almirall. Multimorbidity and quality of life: a closer look. Health Qual. Life Outcomes, 2007;5(52).

3. M. Hunger, B. Thorand, M. Schunk, A. Doring, P. Menn, A. Peters, and R. Holle. Multimorbidity and health-related quality of life in the older population: results from the German KORA-age study. Health Qual. Life Outcomes, 2011;9:53.

4. Z. Fuchs, T. Blumstein, I. Novikov, A. Walter-Ginzburg, M. Lyanders, J. Gindin, B. Habot, and B. Modan. Morbidity, comorbidity, and their association with disability among community-dwelling oldest-old in Israel. J. Gerontol. A. Biol. Sci. Med. Sci., 1998;53(6):447-455.

5. C. Vogeli, A.E. Shields, T.A. Lee, T.B. Gibson, W.D. Marder, K.B. Weiss, and D. Blumenthal. Multiple chronic conditions: prevalence, health consequences, and implications for quality, care management, and costs. J. Gen. Intern. Med., 2007;22 (3):391-395.

6. L.G. Glynn, J.M. Valderas, P. Healy, E. Burke, J. Newell, P. Gillespie, and A.W. Murphy. The prevalence of multimorbidity in primary care and its effect on health care utilization and cost. Fam. Pract., 2011;28(5):516-523.

7. R. Gijsen, N. Hoeymans, F.G. Schellevis, D. Ruwaard, W.A. Satariano, and G.A. van den Bos. Causes and consequences of comorbidity: a review. J. Clin. Epidemiol., 2001;54(7):661-674.

8. P. Bower, W. Macdonald, E. Harkness, L. Gask, T. Kendrick, J.M. Valderas, C. Dickens, T. Blakeman, and B. Sibbald. Multimorbidity, service organization and clinical decision making in primary care: a qualitative study. Fam. Pract., 2011;28(5):579-587.

9. S. Aarts, M. van den Akker, F.E. Tan, F.R. Verhey, J.F. Metsemakers, and M.P. van Boxtel. Influence of multimorbidity on cognition in a normal aging population: a 12-year follow-up in the Maastricht Aging Study. Int. J. Geriatr. Psychiatry, 2011;26(10):1046-1053.

10. S. Aarts, M. van den Akker, K.J. Hajema, A.M. van Ingen, J.F. Metsemakers, F.R. Verhey, and M.P. van Boxtel. Multimorbidity and its relation to subjective memory complaints in a large general population of older adults. Int. Psychogeriatr., 2011;23(4):616-624.

11. A. Marengoni, S. Angleman, R. Melis, F. Mangialasche, A. Karp, A. Garmen, B. Meinow, and L. Fratiglioni. Aging with multimorbidity: A systematic review of the literature. Ageing Res Rev, 2011;10(4):430-439.

12. M.P. van Boxtel, F. Buntinx, P.J. Houx, J.F. Metsemakers, A. Knottnerus, and J. Jolles. The relation between morbidity and cognitive performance in a normal aging population. J. Gerontol. A. Biol. Sci. Med. Sci., 1998;53(2):147-154.

13. R.W. Ponds, K.J. Commissaris, and J. Jolles. Prevalence and covariates of subjective forgetfulness in a normal population in The Netherlands. Int. J. Aging Hum. Dev., 1997;45(3):207-221.

14. J. Banaszak-Holl, A.M. Fendrick, N.L. Foster, A.R. Herzog, M.U. Kabeto, D.M. Kent, W.L. Straus, and K.M. Langa. Predicting nursing home admission: estimates from a 7-year follow-up of a nationally representative sample of older Americans. Alzheimer Dis. Assoc. Disord., 2004;18(2):83-89.

15. A. Ott, R.P. Stolk, F. van Harskamp, H.A. Pols, A. Hofman, and M.M. Breteler. Diabetes mellitus and the risk of dementia: The Rotterdam Study. Neurology, 1999;53(9):1937-1942.

16. Z. Rihmer and M. Arato. Depression and diabetes mellitus. A study of the relationship between serum cortisol and blood sugar levels in patients with endogenous depression. Neuropsychobiology, 1982;8(6):315-318.

17. E.J. Ludman, W. Katon, J. Russo, M. Von Korff, G. Simon, P. Ciechanowski, E. Lin, T. Bush, E. Walker, and B. Young. Depression and diabetes symptom burden. Gen. Hosp. Psychiatry, 2004;26(6):430-436. 
18. J. Iavindrasana, G. Cohen, A. Depeursinge, H. Muller, R. Meyer, and A. Geissbuhler. Clinical data mining: a review. Yearbook of Medical Informatics, 2009:121-133.

19. C. Piatetsky-Shapiro. Knowledge Discovery in Real Databases: A Report on the IJCAI-89 Workshop. Artificial Intelligence Magazine, 1991;11(5):68-70.

20. U. Fayyad, G. Piatetsky-Shapiro, and P. Smyth. From Data Mining to Knowledge Discovery in Databases. Artificial Intelligence Magazine, 1996;17(3):37-54.

21. J.H. Friedman, Data Mining and Statistics. What's the Connection?, in Proc. of the 29th Symposium on the Interface: Computing Science and Statistics. 1997: Houston, Texas.

22. J.D. Hand. Data mining: statistics and more? . The American Statistician, 1998;52:112-118.

23. A. Silberschatz and A. Tuzhilin. What Makes Patterns Interesting in Knowledge Discovery Systems. IEEE Transactions on Knowledge and Data Engineering, 1996;8(6):970-974.

24. A. Wong, H.C. Boshuizen, F.G. Schellevis, G.J. Kommer, and J.J. Polder. Longitudinal administrative data can be used to examine multimorbidity, provided false discoveries are controlled for. J. Clin. Epidemiol., 2011;64(10):1109-1117.

25. M. Fortin, H. Soubhi, C. Hudon, E.A. Bayliss, and M. van den Akker. Multimorbidity's many challenges. BMJ, 2007;334(7602):1016-1017.

26. C. Diederichs, D.B. Bartels, and K. Berger. Methodological challenges concerning the selection of diseases for a standardized multimorbidity index. Bundesgesundheitsblatt Gesundheitsforschung Gesundheitsschutz, 2011;54(8):972-978.

27. J.M. Guralnik. Assessing the impact of comorbidity in the older population. Ann. Epidemiol., 1996;6(5):376-380.

28. M. van den Akker, F. Buntinx, J.F. Metsemakers, S. Roos, and J.A. Knottnerus. Multimorbidity in general practice: prevalence, incidence, and determinants of co-occurring chronic and recurrent diseases. J. Clin. Epidemiol., 1998;51(5):367-375.

29. J.F.M. Metsemakers, P. Hoppener, J.A. Knottnerus, R.J. Kocken, and C.B. Limonard. Computerized health information in The Netherlands: a registration network of family practices. Br. J. Gen. Pract., 1992;42(356):102-106.

30. J.F. Metsemakers, J.A. Knottnerus, G.J. van Schendel, R.J. Kocken, and C.B. Limonard. Unlocking patients' records in general practice for research, medical education and quality assurance: the Registration Network Family Practices. Int. J. Biomed. Comput., 1996;42(1-2):43-50.

31. H. Beukema-Siebenga, To go or not to go. An explorative study on people who seldom consult a general practitioner. 1995: Groningen. [Thesis].

32. J.J. Jolles, P.J. Houx, M.P.J. van Boxtel, and R.W.H.M. Ponds. The Maastricht Aging Study: Determinants of cognitive aging. 1995, Maastricht: Neuropsych Publishers Maastricht.

33. J. Jolles, M.P. van Boxtel, R.W. Ponds, J.F. Metsemakers, and P.J. Houx. The Maastricht aging study (MAAS). The longitudinal perspective of cognitive aging. Tijdschrijft voor Gerontologie en Geriatrie, 1998;29(3):120-129.

34. T.B. Harris, L.J. Launer, G. Eiriksdottir, O. Kjartansson, P.V. Jonsson, G. Sigurdsson, G. Thorgeirsson, T. Aspelund, M.E. Garcia, M.F. Cotch, H.J. Hoffman, and V. Gudnason. Age, Gene/Environment Susceptibility-Reykjavik Study: multidisciplinary applied phenomics. Am. J. Epidemiol., 2007;165(9):1076-1087.

35. R. O'Brien, S. Wyke, B. Guthrie, G. Watt, and S. Mercer. An 'endless struggle': a qualitative study of general practitioners' and practice nurses' experiences of managing multimorbidity in socioeconomically deprived areas of Scotland. Chronic Illn., 2011;7(1):45-59.

36. C.J. Lacey, M.R. Salzberg, H. Roberts, T. Trauer, and W.J. D'Souza. Psychiatric comorbidity and impact on health service utilization in a community sample of patients with epilepsy. Epilepsia, 2009;50(8):1991-1994.

37. M. Fortin, G. Bravo, C. Hudon, L. Lapointe, M.F. Dubois, and J. Almirall. Psychological distress and multimorbidity in primary care. Ann. Fam. Med., 2006;4(5):417-422. 


\section{Chapter 9}

38. C.M. Boyd, J. Darer, C. Boult, L.P. Fried, L. Boult, and A.W. Wu. Clinical practice guidelines and quality of care for older patients with multiple comorbid diseases: implications for pay for performance. JAMA, 2005;294(6):716-724.

39. J.L. Wolff, B. Starfield, and G. Anderson. Prevalence, expenditures, and complications of multiple chronic conditions in the elderly. Arch. Intern. Med., 2002;162(20):2269-2276.

40. J.A. Knottnerus. Role of the electronic patient record in the development of general practice in The Netherlands. Methods Inf. Med., 1999;38(4-5):350-354.

41. C.c.o. WONCA. ICPC International Classification of Primary Care. 1987, Oxford: Oxford University Press.

42. M. van den Akker, F. Buntinx, and J. Knottnerus. Comorbidity or multimorbidity: What's in a name. A review of literature. Eur. J. Gen. Pract., 1996;2:65-70.

43. J. Neeleman, J. Ormel, and R.V. Bijl. The distribution of psychiatric and somatic III health: associations with personality and socioeconomic status. Psychosom. Med., 2001;63(2):239-247. 
CHAPTER 10

Summary

Samenvatting

Dankwoord

About the author

Publications 
Forecasts suggest that, in the upcoming decades, the percentage of people with multimorbidity will increase dramatically in the Netherlands. This dramatic increase is primarily due to changes in wealth indicators (e.g. improved nutrition and hygiene) which have resulted in increased longevity and an increasing number of older individuals. Consequently, general practitioners are increasingly confronted with the management of individuals with multimorbidity.

The general background, aims and research questions of the studies included in this thesis are described in chapter 1 . The aim of this thesis is twofold; 1) to report on several adverse health-related factors and related constructs and 2) to introduce and apply innovative research strategies useful to analyzing large amounts of medical data.

\section{MULTIMORBIDITY: ADVERSE HEALTH AFFECTS AND RELATED CONSTRUCTS}

Chapter 2 and 3 describe the relationship between multimorbidity and (subjective) cognitive functioning. The study presented in chapter 2 investigates the association between multimorbidity and subjective memory complaints. This cross-sectional study was based on data obtained from a postal survey designed by the PublicHealth Service (Gemeentelijke Gezondheidsdienst, GGD) concerning 15,188 individuals aged 55 years and over, living independently in Limburg, the Netherlands. Multimorbidity was not only related to subjective memory problems but was also related to the degree of worrying about memory complaints in people who perceived themselves as forgetful. Multimorbidity was also related to a larger increase in these subjective memory complaints during the last year. In addition, individuals with multimorbidity who were aged 55 to 69 years reported more subjective memory complaints compared to individuals with multimorbidity who were aged 69 and over. Men who perceived themselves as forgetful and who suffered from multimorbidity reported a larger increase in their memory problems during the past year compared to their female counterparts. Psychological distress appeared to be related to all three subjective memory-related outcome measures.

In chapter 3, the effect of multimorbidity on cognition in a normal healthy aging population was investigated. Data were collected as part of the Maastricht Aging Study (MAAS), a prospective study into the determinants of cognitive aging. Eligible MAAS participants $(\mathrm{N}=1763)$, who were 24-81 years of age, were recruited from the Registration Network Family Practices (RNH) which enabled the use of medical records of all participants. The association between 96 chronic diseases, grouped into 23 disease clusters, and cognition at baseline, at 6 and 12 years of follow-up, were analyzed. Cognitive performance was measured in two main domains: verbal memory and psychomotor speed. Multiple disease clusters appeared to be associated with cognitive functioning. The disease cluster combination malignancies and movement disorders also appeared to significantly affect cognition. Nevertheless, these effects of disease clusters and combinations of two disease clusters and on side and cognition on the other appeared to be relatively small in a normal aging population. 
Multimorbidity is well known for its profound negative effect on mental and physical functioning. Nevertheless, it remains unclear if these effects are temporary or persistent. Chapter 4 describes a study which investigated if the effects of single morbidity (i.e. a single disease within one individual) and multimorbidity on one side and physical and mental functioning on the other, are stable over time. Eligible participants from the MAAS $(\mathrm{N}=1184)$, 24-81 years old, were originally recruited from the Registration Network Family Practices. The Short Form Health Survey (SF-36) was used to assess physical and mental functioning. In contrast to single morbidity, the adverse effects of multimorbidity on physical functioning were persistent over time. At baseline, individuals who acquire one or more medical conditions during 3-years follow-up, already reported more decline in physical functioning than individuals who remained healthy during these years. The results of ad-hoc analyses in which the SCL-90 anxiety and depression scales were used, showed that multimorbidity indeed was related to increased levels of depressive and anxiety complaints. Nevertheless, these psychological complaints, caused by multimorbidity, appeared to decline over time.

Chapter 5 presents a study conducted at the National Institute on Aging, part of the National Institutes of Health in Bethesda, Maryland, USA. This study, based on a large population-based sample of 4,414 older individuals, aged 70 and older and living in Iceland, investigated heterogeneity in the frail older population. The first aim of this study was to estimate the overall prevalence of frailty as well as to estimate the co-occurrence of disability and multimorbidity within the frail population. The second aim was to characterize subpopulations of frailty using various quantitative physiological markers. The final aim was to examine the risk for mortality and nursing home admission in subpopulations of frail older adults.

Frailty was defined by having three or more of the following characteristics: weight loss, weakness, low energy levels, slowness and low physical inactivity. Multimorbidity was based on thirteen highly prevalent conditions. Disability was assessed by five activities of daily living (e.g. problems with bathing and walking). The following five subpopulations were formed: 1) Non-frail (reference group); 2) Frail only; 3) Frail with disability (FD); 4) Frailty with multimorbidity (FM); 5) Frail with disability and multimorbidity (FMD).

Frailty appeared to be highly prevalent in the Icelandic population. Although frailty is often accompanied by multimorbidity and disability, nearly $13.5 \%$ of the frail individuals were frail without having multimorbidity and/or disability. Compared to non-frail individuals, the frail only group showed decreases in cognitive functioning and increased levels of inflammation. The other three frail subpopulations did not only showed significantly decreases in cognition and increased levels of inflammation but also showed a higher incidence of white matter lesions, and increased levels of calcium, glucose and red blood cell distribution width. Although frailty was associated with an increased risk for mortality and nursing home admission, these risks substantially differed between frail subpopulation. When evaluating the risk for mortality and nursing home admission in different subpopulations of frailty, the results indicated that, while mortality was primarily driven by the presence of multimorbidity (i.e. diseases), disability appeared to be the 
driving force behind nursing home admission. As a result, the 'only frail' group, i.e. frail individuals who have no evidence of multimorbidity and disability, was not at an increased risk for mortality and nursing home admission. Hence, the adverse health risks associated with frailty in the general older adult population may primarily be driven by multimorbidity and disability.

\section{Diabetes Mellitus and Depression}

In chapter 6, the longitudinal association between diabetes mellitus type II and depression was investigated. This study was carried out within the context of the RNH. Patients diagnosed with diabetes mellitus at or after the age of 40 were compared with age matched controls without a history of diabetes. Both groups were followed for an emerging first diagnosis of depression and/or depressive feelings with a mean follow-up time of 7.7 years. In total, $2.0 \%$ of the people diagnosed with diabetes mellitus developed a depressive disorder, compared to $1.6 \%$ of the reference group. Patients with diabetes mellitus were more likely to develop subsequent depression than persons without a history of diabetes. Results from this large longitudinal study based on a general practice population indicate that the association between diabetes mellitus type II and depression is weaker than previously found in cross-sectional research using self-report surveys.

\section{INNOVATIVE RESEARCH STRATEGIES}

\section{Knowledge Discovery in Databases}

Chapter 7 describes, based on the available literature, the theory and practical applications of Knowledge Discovery in Databases (KDD). The aim of this manuscript was to get the medical researcher acquainted with this emerging field. KDD is aimed at extracting previously unknown, hidden and potentially useful information and incorporates a new generation analyzing methods that is able to explore and model large amounts of (bio)medical data and documents in a (semi)automatically manner.

Although KDD approaches are not intended to replace traditional statistical analyzing techniques, they may help (bio)medical researchers in the practice of exploratory research (i.e. research aimed at hypotheses generation). Rather than to serve as a golden standard, the application of KDD is aimed at supporting the knowledge and ideas of medical experts. This manuscript described in this chapter discusses several interesting applications of KDD that are useful in the practice of hypothesis generating in epidemiology and (bio)medical research.

\section{Patterns of Multimorbidity}

The study presented in chapter 8 presents a study on the identification of novel, interesting combinations of psychiatric and somatic diseases. Six conditions, coded in ICPC chapter P (Psychological), were involved: affective psychosis (P73), depressive disorder (P76), schizophrenia (P72), mental retardation (P85), personality disorder (P80) and suicide attempt (P77). The following ICPC chapters with somatic conditions were included: D (Gastrointestinal), K (Circulatory), L (Musculoskeletal), N (Neurological), R (Respiratory) 
and $\mathrm{T}$ (Endocrine, metabolic and nutritional). In total, 166 disease combinations (i.e. consisting of one medical condition from ICPC chapter P and one medical condition from one of the somatic ICPC chapters) were selected for further evaluation. We focused on disease combinations with a prevalence of $\geq 10$ (i.e. as assessed by The Registration Network Family Practices; $N=87,837$ ) and an observed/expected ratio of $\geq 1.5$. In total, 37 disease combinations with a prevalence of $\geq 10$ and an observed/expected ratio of $\geq 1.5$ were evaluated by three experts. The following five disease combinations were discussed in detail during an expert meeting; appendicitis and personality disorder, uncomplicated hypertension and psychotic affective disorders, migraine disorder and personality disorder, pulmonary emphysema and depressive disorder, and lipid metabolism and depressive disorder. These novel and potential relevant disease combinations need to be confirmed in other, preferable general practice based, datasets. Subsequently, these disease combinations can be analyzed in cross-sectional or longitudinal studies in order to investigate the plausibility of underlying (causal) relationships. In addition, the knowledge discovery method described in this chapter illustrates the use of innovative research strategies in order to identify prevalent disease combinations. Hence, the present study promotes hypothesisdriven studies in the field of multimorbidity.

\section{GENERAL DISCUSSION}

Chapter 9 provides a general discussion of this thesis by discussing its landmarks and pitfalls with a specific focus on methodological considerations. The definition and assessment of multimorbidity used in this thesis is described even as the consequences hereof. Moreover, the quality of the data is discussed as well as the use of (innovative) analyzing strategies. Finally, this chapter links the results from the seven research projects to daily clinical practice as well as to future research in the field of multimorbidity.

Although each study in this thesis has resulted in several specific conclusions, the following general conclusions can be drawn:

- Multimorbidity is highly prevalent.

- People with multimorbidity report more subjective memory complaints than their healthy counterparts.

- Multiple disease clusters have an adverse affect on cognitive functioning. Nevertheless, these affects appears to be small in a normal aging population.

- Multimorbidity, disability and frailty should be perceived as distinct concepts, since each concept appears to be related differently to quantitative biological markers and adverse health outcomes.

- The adverse healthy risks associated with frailty may primarily be driven by disease burden and disability.

- While mental problems related to multimorbidity appear to decrease over time, the decline in physical functioning caused by multimorbidity appears to be permanent. 
- Knowledge Discovery in Databases can aid medical researchers in their search for patterns and association (e.g. patterns of multimorbidity) in large amounts of medical data or medical text.

To address the above-mentioned conclusions and problems, the following recommendations are postulated:

- Identifying unknown patterns of multimorbidity is particularly important for future research since knowledge on frequently occurring disease combinations facilitate and accelerates disease diagnosis. The extent to which frequently occurring disease combinations represent general disease susceptibility also deserves future exploration.

- Epidemiologic research is needed to develop a risk profile for multimorbidity. This risk profile can direct future intervention programs, aimed at increasing the quality of life of people with multimorbidity by ameliorating and diminishing its adverse health consequences.

- Health care should move away from a disease specific approach and should move towards an integrated, multidimensional approach of health care, especially when confronted with multimorbidity.

This thesis illustrates that multimorbidity is related to several adverse health effects including a permanent decline in physical functioning. In addition, it underscores that multimorbidity should not be used interchangeably with other gerontological constructs such as disability and frailty. Finally, this thesis demonstrates that innovative research strategies, such as knowledge discovery techniques, can provide novel and potentially relevant medical patterns and associations, including patterns of multimorbidity. 
SAMENVATTING 
Door de vergrijzing van de bevolking en de toenemende levensverwachting zal het aantal personen met twee of meer chronische aandoeningen, ofwel multimorbiditeit, in de toekomst nog vaker voorkomen. De impact van multimorbiditeit is groot. Zo blijken mensen met multimorbiditeit niet alleen een verhoogde kans op lichamelijke beperkingen te hebben, maar ook op mentale problemen, hospitalisatie en zelfs sterfte. Tevens blijkt uit onderzoek dat het risico op deze uitkomsten groter wordt naarmate het aantal ziektes van een persoon toeneemt. Daarnaast kan de zorg voor mensen met multimorbiditeit een grote invloed hebben op het dagelijks leven van familie en vrienden.

Mensen met multimorbiditeit maken vaker en meer gebruik van gezondheidszorgvoorzieningen. Zo bezoeken zij vaker de huisarts of een medisch specialist en zijn er meer zorgverleners betrokken bij mensen die multimorbiditeit hebben. Patiënten met multimorbiditeit stellen hun huisarts voor verschillende uitdagingen. Multimorbiditeit bemoeilijkt namelijk niet alleen het diagnostisch proces naar nieuwe chronische ziektes maar compliceert ook de behandeling van de ziektes. Zo worden mensen met multimorbiditeit dikwijls geconfronteerd met tegenstrijdige adviezen en neemt het risico op gecontraïndiceerde medicatie toe. Zorg voor mensen met multimorbiditeit is dus intensiever en complexer wat resulteert in hogere gezondheidskosten. Kortom, multimorbiditeit heeft grote gevolgen voor zowel de patiënt, zijn naasten en voor de maatschappij.

Over de definitie en terminologie van multimorbiditeit bestaat tot op heden nog geen consensus. De definitie geïntroduceerd door van den Akker en collega's in 1995, die multimorbiditeit omschrijven als twee of meer chronische aandoeningen, zal in dit proefschrift gehanteerd worden. Een aan multimorbiditeit verwante term is comorbiditeit; de aanwezigheid van een chronische ziekte in combinatie met een index ziekte (bijv. depressieve stoornis in mensen met diabetes mellitus type II). Daarnaast wordt multimorbiditeit regelmatig in een adem genoemd met andere geriatrische concepten zoals 'frailty' (dwz. broosheid en fragiliteit onder ouderen mensen) en 'disability' (dwz. het onvermogen om bepaalde activiteiten uit het dagelijkse leven uit te voeren).

\section{ONDERZOEKSDOELSTELLINGEN- EN VRAGEN}

Hoofdstuk 1 leidt de centrale doelstelling van dit proefschrift in. Dit proefschrift heeft twee doelstellingen en wel de volgende: 1) het in kaart brengen van een aantal negatieve gezondheidseffecten en gerelateerde constructen van multimorbiditeit en 2) het introduceren en toepassen van nieuwe analyse methodes bruikbaar voor het analyseren van grote hoeveelheden medische data.

De bovenstaande twee doelstellingen leiden de volgende, meer gedetailleerde, onderzoekvragen in:

- Houdt multimorbiditeit verband met subjectieve geheugenklachten?

- Wat is het longitudinale effect van multimorbiditeit op het cognitief functioneren? 
- Is de relatie tussen fysieke en mentale gezondheid enerzijds en multimorbiditeit anderzijds tijdelijk of permanent?

- Wat is de relatie tussen multimorbiditeit, frailty en disability?

- Is diabetes mellitus type II gerelateerd aan depressie in een populatie van eerstelijns patiënten?

- Is Knowledge Discovery in Databases (KDD) bruikbaar voor medisch onderzoek?

- Welke patronen van multimorbiditeit komen vaker voor bij patiënten dan verwacht op basis van toeval?

\section{DE NEGATIEVE EFFECTEN VAN MULTIMORBIDITEIT}

\section{Multimorbiditeit en Cognitief Functioneren}

Hoofdstuk 2 beschrijft een cross-sectioneel onderzoek naar de relatie tussen multimorbiditeit en subjectieve geheugen klachten. Het onderzoek, gebaseerd op een schriftelijke vragenlijst opgesteld door de Gemeentelijke Gezondheids Dienst (GGD), vond plaats onder 15.188 55-plussers die ten tijden van het onderzoek zelfstandig woonden in Zuid-Limburg, Nederland. Multimorbiditeit bleek voorspellend voor de mate van zorgen die mensen hebben over hun geheugenklachten; mensen met multimorbiditeit rapporteerden meer geheugenklachten dan hun gezonde leeftijdsgenoten. Ook bleek uit deze studie dat multimorbiditeit een belangrijke voorspeller is voor het rapporteren van een toename van geheugen klachten gedurende het afgelopen jaar. Dit verband bleek meer sterker voor mannen dan voor vrouwen. Mensen met multimorbiditeit die ook nog psychische klachten hadden, rapporteerden significant meer geheugen klachten dan mensen met multimorbiditeit zonder psychische klachten.

In hoofdstuk 3 wordt een studie gepresenteerd gericht op de relatie tussen multimorbiditeit en cognitie. Personen tussen de 24 en 81 jaar $(\mathrm{N}=1.763)$ werden geselecteerd uit het RegistratieNet Huisartspraktijken (RNH) voor deelname aan de Maastricht Aging Study (MAAS), een longitudinale studie gericht op het in kaart brengen van de determinanten van normale cognitieve veroudering. De longitudinale relatie tussen 96 chronische ziektes, gegroepeerd in 23 clusters, en cognitief functioneren werd onderzocht. Cognitief functioneren werd gemeten in twee domeinen: verbaal geheugen en informatieverwerkingssnelheid. Multimorbiditeit was aanwezig bij meer dan 55\% van de studie populatie. Verscheidene ziekte clusters hadden een negatief effect op cognitief functioneren. Mensen met multimorbiditeit die leden aan een combinatie van maligniteiten en bewegingsstoornissen toonde een verslechtering in cognitief functioneren. Echter, al deze negatieve effecten lijken relatief klein in een een normale populatie.

\section{Multimorbiditeit en Functionele Gezondheid}

De studie die beschreven wordt in hoofdstuk 4 onderzoekt of de relatie tussen mentale en fysieke gezondheid enerzijds en multimorbiditeit anderzijds tijdelijk of permanent van aard is. Personen tussen de 24 en 81 jaar $(n=1.184)$ werden uit het RegistratieNet 
Huisartspraktijken (RNH) geselecteerd voor deelname aan de Maastricht Aging Study (MAAS). De morbiditeit status (dwz. gezond, enkelvoudige morbiditeit, het hebben van één chronische ziekte, en multimorbiditeit) van de MAAS deelnemers werd op baseline en op 3 en 6 jaar follow-up in kaart gebracht. De Rand-36, een vragenlijst gericht op het in kaart brengen van functionele gezondheid, werd op dezelfde meetmomenten afgenomen. Op baseline, maar niet na 3 en 6 jaar follow-up, bleken deelnemers met enkelvoudige morbiditeit een slechtere fysieke gezondheid te rapporteren dan gezonde deelnemers. Op elke meetmoment rapporteerde mensen met multimorbiditeit een slechtere fysieke gezondheid dan gezonde deelnemers. Daarnaast nam de fysieke gezondheid van deze deelnemers verder af naarmate de tijd vorderde. Zowel single morbiditeit als multimorbiditeit bleek niet gerelateerd aan mentale gezondheid. Deelnemers die in 3 jaar een verandering in morbiditeitsstatus doormakkte (dwz. van gezond naar enkelvoudige morbiditeit of van gezond naar multimorbiditeit), rapporteerde een slechtere fysieke gezondheid dan mensen die gezond bleven. Post-hoc analyses waarin gebruik werd gemaakt van een andere vragenlijst, de Symptom Checklist-90 (SCL-90), toonde wel degelijk een relatie tussen multimorbiditeit en gevoelens van angst en depressie. Deze gevoelens bleken echter af te nemen naarmate de tijd vorderde.

\section{Multimorbiditeit, 'Frailty' en 'Disability'}

Hoofdstuk 5 beschrijft een onderzoek dat is uitgevoerd bij National Institute of Aging, onderdeel van National Institute of Health in Bethesda, USA en is gebaseerd op 4.414 personen, tussen de 77 tot 100 jaar oud, woonachtig in IJsland. De doelstelling van dit onderzoek was drieledig: 1) het vaststellen van de prevalentie van frailty als mede de prevalentie van frailty in combinatie met multimorbiditeit en disability, 2) het karakteriseren van de verschillende frailty-groepen door middel van kwantitatieve fysiologische markers en 3) het vaststellen van het risico van de verschillende groepen op opname in een verpleeghuis en overlijden. Frailty was gedefinieerd als het hebben van drie of meer van de volgende karakteristieken: gewichtsverlies, verminderde kracht, verminderd energie niveau, traagheid en fysieke inactiviteit. Dertien chronische ziektes werden geïncludeerd om multimorbiditeit vaststellen. Multimorbiditeit was gedefinieerd als het hebben van twee of meer chronische aandoeningen. Disability werd geoperationaliseerd door vijf vragen die betrekking hadden op verscheidene activiteiten in het dagelijks leven (bijv. Hebt u moeite met het lopen van een kamer naar de andere). De volgende vijf frailty groepen werden gevormd: 1) niet frail (de controle groep), 2) alleen frail (zonder disability en/of multimorbiditeit), 3) frail met disability, 4) frail met multimorbiditeit en 5) frail met disability en multimorbiditeit.

Frailty bleek vaak voor te komen in de IJslandse populatie. Hoewel frailty vaak in combinatie met multimorbiditeit en disability voorkomt, zijn er ook mensen die alleen frail zijn. Vergeleken met mensen die niet frail zijn, tonen mensen die 'alleen frail' zijn een verminderd cognitief functioneren, meer witte stof letsel en een verhoogd inflammatieniveau. De andere drie frailty-groepen vertoonden niet alleen een verminderd cognitief functioneren, meer witte-stofschade en een verhoogd inflammatie niveau maar lieten daarnaast ook nog een verhoging in calcium, glucose en distributiebreedte van de rode 
bloedcellen zien. Hoewel mensen met frailty een verhoogd risico op opname in een verpleegtehuis en overlijden vertoonde, toonde de 'alleen frail' groep geen verhoogde risico op opname of overlijden. Terwijl de groep 'frailty met disability' alleen een verhoogde kans op opname in een verpleegtehuis had, liet de 'frailty met multimorbiditeit' groep alleen een verhoogd risico op overlijden zien. Terwijl chronische ziektes primair ten grondslag bleken te liggen aan overlijden, bleek een verhoogd risico op opname in een verpleegtehuis voornamelijk veroorzaakt te worden door disability.

\section{Diabetes Mellitus type II en Depressie}

Hoofdstuk 6 beschrijft de relatie tussen diabetes mellitus type II en depressie gebaseerd op data van het RegistratieNet Huisartspraktijken (RNH). Van de 6.140 patiënten die gediagnosticeerd waren met diabetes mellitus ontwikkelde $2 \%$ een depressieve stoornis. Van de 18.416 personen zonder diabetes mellitus ontwikkelde 1,6\% een depressieve stoornis. Kortom, personen met diabetes mellitus blijken een groter risico te hebben op een depressieve stoornis dan mensen die niet gediagnosticeerd zijn met diabetes mellitus. Dit effect kon niet worden verklaard door leeftijd, geslacht of de aanwezigheid van andere chronische aandoeningen. Echter het verband tussen diabetes mellitus en depressie bleek minder groot dan beschreven in eerder, cross-sectioneel en/of vragenlijst onderzoek.

\section{INNOVATIEVE ANALYSE METHODES}

Met de opkomst van de computer is de laatste decennia de hoeveelheid beschikbare medische informatie drastisch toegenomen. Zo bestaan er tegenwoordig grote elektronisch patiëntendossiers (EPD) waarin zorgverleners relevante medische gegevens kunnen opslaan. De grote hoeveelheid medische data heeft er toe geleid dat nieuwe methoden nodig zijn om deze gegevens te kunnen analyseren.

\section{Knowledge Discovery in Databases}

Hoofdstuk 7 introduceert Knowledge Discovery in Databases (KDD), het proces van kennis-extractie uit data of tekst. KDD is gericht op het extraheren van voorheen onbekende, verborgen en mogelijk nuttige informatie. KDD is een nieuwe generatie analysemethoden die in staat om betekenis te geven aan grote hoeveelheden (bio) medische gegevens in een (semi) automatisch manier. Hoewel KDD niet tot doelstelling heeft de traditionele statistische analyse technieken te vervangen, kan het (bio) medische onderzoekers helpen in exploratief onderzoek (dwz. onderzoek gericht op het generen van hypotheses). In plaats van te dienen als gouden standaard, is de toepassing van KDD gericht op het ondersteunen van de kennis en ideeën van medische experts. Het artikel beschreven in dit hoofdstuk bespreekt een aantal interessante toepassingen van KDD die van nut kunnen zijn in exploratief medisch onderzoek.

\section{Clusters van Chronische Aandoeningen}

Hoofdstuk 8 presenteert een studie die gericht is op de identificatie van nieuwe, potentieel relevante combinaties van psychiatrische en somatische aandoeningen. De volgende zes 
aandoeningen, gecodeerd in ICPC hoofdstuk P (Psychologische), werden geïncludeerd: affectieve psychose (P73), depressieve stoornis (P76), schizofrenie (P72), mentale retardatie (P85), persoonlijkheidsstoornis (P80) en poging tot zelfmoord (P77). De volgende ICPC hoofdstukken met somatische aandoeningen werden opgenomen: D (Gastro-intestinaale), K (Cardiovasculair), L (Bewegingsapparaat), N (Neurologische), R (Respiratoir) en $\mathrm{T}$ (Endocriene, metabool en nutritioneel). In totaal werden 166 ziekte combinaties (dwz. een medische aandoening uit ICPC hoofdstuk P en een medische aandoening uit een van de zes somatische ICPC hoofdstukken) geselecteerd voor verdere evaluatie. We concentreerden ons op ziekte combinaties met een prevalentie van $\geq 10$ (geregistreerd in het RegistratieNet Huisartspraktijken $(\mathrm{RNH}) ; \mathrm{N}=87.837$ ) en met een observed/expected ratio van $\geq 1,5$. In totaal werden 37 ziektecombinaties met een prevalentie van $\geq 10$ en een observed/expected ratio van $\geq 1,5$ geëvalueerd door drie experts. De volgende vijf ziekte combinaties werden uitvoerig besproken tijdens een expert bijeenkomst: appendicitis en persoonlijkheidsstoornis; ongecompliceerde hypertensie en psychotische affectieve stoornis; migraine en persoonlijkheidsstoornis; emfyseem en depressieve stoornis; en vetstofwisselingsstoornis en depressieve stoornis. Deze nieuwe en potentiële relevante ziekte combinaties dienen te worden bevestigd in onafhankelijke, bij voorkeur huisartsgeneeskundige, datasets. Nadien kunnen deze ziekte combinaties worden geanalyseerd in cross-sectioneel of longitudinale studies om de plausibiliteit van een onderliggende (causale) relatie te onderzoeken. Daarnaast illustreert de toegepaste KDD methode het gebruik van innovatieve analyse methodes om frequente ziekte combinaties te identificeren.

\section{ALGEMENE DISCUSSIE}

In de algemene discussie, hoofdstuk 9, worden de belangrijkste bevindingen en conclusies van de acht verschillende onderzoekprojecten samenvattend besproken om zo de vraagstellingen van dit proefschrift te beantwoorden. De methodologische overwegingen van deze studies worden besproken waarin zowel sterktes als zwaktes worden gepresenteerd. Daarnaast wordt de operationalisatie van multimorbiditeit beschreven en de mogelijke implicaties hiervan. Tevens wordt de kwaliteit van de gebruikte databestanden besproken en geëvalueerd evenals de diverse analyse methodes. Ten slotte worden aanbevelingen voor klinisch beleid en toekomstig onderzoek geformuleerd.

Hoewel elke afzonderlijke studie in dit proefschrift resulteert in specifieke conclusies, kunnen de volgende algemene conclusies worden getrokken:

- Multimorbiditeit is, in de algemene bevolking, eerder regel dan uitzondering.

- Vergeleken met gezonde mensen, rapporteren mensen met multimorbiditeit meer geheugen klachten.

- Mensen met multimorbiditeit vertonen ook in afname in cognitief functioneren. Echter, deze afname blijkt klinisch weinig relevant.

- Hoewel multimorbiditeit, 'disability' en 'frailty' aan elkaar gerelateerde concepten zijn, moeten ze wel degelijk als aparte constructen worden beschouwd met ieder een eigen risico op opname in een verpleegtehuis en overlijden. 
- Multimorbiditeit lijkt geassocieerd te zijn met een permanente vermindering in fysieke gezondheid. De mentale problemen die gerelateerd zijn aan multimorbiditeit lijken na verloop van tijd te verminderen.

- De combinatie van medische data verzameld in de RegistratieNet Huisartsenpraktijk leent zich bij uitstek voor verder onderzoek naar multimorbiditeit.

- Knowledge Discovery in Databases kan onderzoekers helpen bij het zoeken naar relaties en patronen in grote hoeveelheden medische data.

Gezien de bovenstaande conclusies en problemen worden de volgende aanbevelingen gedaan:

- Onderzoek naar het identificeren van frequent voorkomende ziekte combinaties (dwz. patronen van multimorbiditeit) is van belang aangezien dit het diagnostisch proces van ziektes kan vergemakkelijken en versnellen. De mate waarin frequent voorkomende ziekte combinaties een algemene ziektegevoeligheid weerspiegelen, dient ook nader onderzocht te worden.

- Etiologisch onderzoek gericht op het in kaart brengen van risico factoren van multimorbiditeit kan leiden tot een risicoprofiel voor multimorbiditeit. Dit risicoprofiel kan richting geven aan adequate interventieprogramma's die tot doel hebben de vermindering in kwaliteit van leven van patiënten met multimorbiditeit tegen te gaan.

- De huidige opzet van zorg die zich voornamelijk richt op specifieke, individuele chronische aandoeningen, kan leiden tot versnipperde en gefragmenteerde gezondheidszorg voor mensen met multimorbiditeit. Integrale zorg voor de individuele zorgvrager met multimorbiditeit is daarom een belangrijk aandachtspunt.

Dit proefschrift toont dat multimorbiditeit gerelateerd is an diverse negatieve gezondheidseffecten waaronder een permanente afname in fysieke gezondheid. Daarnaast onderstreept dit proefschrift dat multimorbiditeit niet verward dient te worden met andere geriatrische constructen zoals frailty en disability. Tot slot toont dit proefschrift aan dat innovatieve analyse methodes, nieuwe en potentieel relevante medische patronen en verbanden kunnen identificeren in grote hoeveelheden medische data. 
DANKWOORD 
Aan de totstandkoming van dit proefschrift hebben, zoals bij elke uitdagende en langdurige taak het geval is, veel mensen een bijdrage geleverd. Op de komende pagina's zou ik graag van de gelegenheid gebruik maken deze mensen te bedanken die, ieder op hun geheel eigen manier, de afgelopen vier jaar een belangrijke rol in mijn leven hebben gespeeld. Ik wil dit doen aan de hand van de volgende indeling; promotie project, overige collega's, familie en vrienden. Dit wil echter niet zeggen dat mensen niet tot twee of drie groepen kunnen behoren Het behoren tot vier groepen acht ik echter uitgesloten; zover ik weet is geen enkel familielid van mij direct betrokken geweest bij mijn $\mathrm{PhD}$ project.

\section{Promotie project}

Ik zou graag willen beginnen met die twee mensen te bedanken die niet alleen een enorm grote bijdrage hebben geleverd aan de ontwikkeling van mijn proefschrift, maar ook aan mijn ontwikkeling als mens. Beste Marjan van den Akker, jouw vrolijkheid en expertise op (zo lijkt het) bijna alle vlakken van wetenschappelijk onderzoek, hebben ervoor gezorgd dat ik de afgelopen vier jaar mijn werk met plezier en interesse heb uitgevoerd. Tevens denk ik met veel plezier terug aan ons etentje in Dubrovnik waar zowel werkgerelateerde als persoonlijke zaken op een leuke, ongedwongen manier besproken werden. Beste Martin van Boxtel, jij hebt mij op een prettige en deskundige wijze geleerd wat het is om een wetenschappelijk onderzoeker te zijn. Ik kon er altijd op vertrouwen dat je mijn stukken rondig en uitermate snel van commentaar voorzag. Dit heeft er voor gezorgd dat het temp altijd behouden bleef. Daarnaast leerde je mij, soms onbewust, een aantal persoonlijke vaardigheden die mij, zowel op werk als op persoonlijk gebied, een beter mens maken. Martin en Marjan, individueel zijn jullie goed, samen zijn jullie uitzonderlijk. Ik voel me bevoorrecht om een dergelijk goede begeleiding tijdens mijn promotie traject te hebben mogen ervaren. Ik kan alleen maar hopen dat dit voor elke promovendus is weggelegd.

Daarnaast wil ik mijn promotoren, die als overkoepelend orgaan hebben gefungeerd, bedanken. Beste Job Metsemakers, jouw visie als praktiserend huisarts heeft mij gewezen op de klinische relevantie van mijn promotie traject. Daarnaast denk ik met veel plezier terug aan de leuke gesprekken over familie en reizen (m.i. delen we de liefde voor Indonesië). Beste Frans Verhey, jouw aanwijzigingen bij het schrijven van artikelen, die voornamelijk betrekking had op de klinische relevantie ervan, waren steeds verfrissend. Jelle Jolles, ondanks dat je aanwezigheid als mijn promotor slechts van zeer korte duur was, wil ik je bedanken voor je bijdrage tijdens de eerste maanden: een goed begin, is het halve werk.

Beste Rein Vos, met behulp van jouw expertise en netwerk heb ik contact kunnen leggen met mensen van de afdeling Biomedische Informatica aan de Erasmus Universiteit in Rotterdam. Hierdoor is mijn proefschrift geworden wat het nu is; een samensmelting van huisartsgeneeskundig onderzoek en knowledge discovery. Beste Hans Bosma, jij was wellicht de stille kracht in onze project groep. Jouw kennis van longitudinale analyses heeft mij geholpen in het uitvoeren van een aantal onderzoeken. Daarnaast hebben we samen het mysterie achter de SF-36 'ontrafeld'. Bedankt voor je suggestie om 'alles simpel te houden' als ik weer eens moeilijke statistische modellen aan het uitdenken was. 
De volgende mensen die ik wil bedanken zijn Frans Tan en Björn Winkens. Jullie hebben mijn statistische vaardigheden naar een hoger niveau getild met behulp van jullie expertise. Met groot enthousiasme ontving ik dikwijls een heldere uitleg van ingewikkelde materie. Tevens wil ik Nico Rozendaal bedanken voor zijn enorme hulp bij het werken met SPSS. Ik heb bewondering voor jouw kennis maar ook voor jou als mens. Jouw vriendelijkheid, behulpzaamheid en goede humeur verschaften me altijd enorm veel vreugde.

I would like to thank all American and Icelandic co-authors for their contribution to the AGES manuscript. Thank you all for your time and effort.

Euvgeni Smirnov, Irmin Auwerda, Georgi Albinov, Erik van Mulligen, en Jan Kors: bedankt voor jullie medewerking aan de data en text mining kant van mijn proefschrift.

Onno van Schayck, Trudy van der Wijden, Hannerieke van der Boom, Rob de Bie, Martijn Berger en Gonnie Klabbers. Ik wil jullie allen hartelijk danken voor het verbreden van mijn 'promovendus horizon'. Door mijn werkzaamheden als promovendus vertegenwoordigster is mij een kijkje gegund in de 'keuken van CAPHRI'. Jullie hebben mij het gevoel gegeven dat alle ideeën en suggesties van harte welkom waren om zo een beter en uitgebreider platform voor promovendi te creëren. Katarina Putnik, Marla Woolderink en Laura van Alphen, jullie wil ik bedanken voor de leuke en hechte samenwerking tijdens deze periode. Ik heb veel plezier beleefd aan onze lunchbijeenkomsten waarin we al etende, brainstormde over workshops, de CAPHRI mail en 'het promovendus zijn'.

Daarnaast zou ik mijn dank willen richten tot alle huisartsen die participeren aan RNH en hun patiënten. Zonder hun continue en precieze registratie, zou dit proefschrift niet zijn zoals het nu is. Tevens een bedankje voor alle patiënten die het geen problemen vinden dat hun gegevens anoniem worden gebruikt voor wetenschappelijk onderzoek.

\section{Collega's}

Ten eerste wil ik mijn dank richten tot alle medewerkers van HAG op de tweede en derde verdieping van die ik hier niet bij allen bij naam noem aangezien jullie in grote getallen aanwezig zijn. Bedankt voor de korte (en lange) praatjes die veelal bij het koffie apparaat of in het trappenhuis plaats vonden; ze waren altijd een welkome afleiding. Ik heb jullie toegankelijkheid altijd enorm gewaardeerd. Jullie hebben ervoor gezorgd dat ik me vanaf dag één welkom voelde!

Daarnaast een bedankje voor het ondersteunend personeel van CAPHRI. Erie, Astrid, Iris, Tanja, Nikki en Margareth bedankt dat jullie altijd tijd hadden voor mijn vragen.

Ik wil ook graag van de gelegenheid gebruik maken om een aantal mensen te bedanken die mij vooral op sociaal vlak hebben bijgestaan tijdens mijn promotie traject. Ik wil beginnen bij Daniel Kotz. Beste Daniel, jij was en bent mijn eerste kamergenoot geweest. Nooit was 
het je te veel om mij van advies te voorzien of me handige tips toe te fluisteren. Door jouw werkervaring te delen met mij, heb je me niet alleen veel geleerd maar ook enorm welkom geheten.

Matthijs, ook jou wil ik even apart bedankt voor je opbeurende, leuke, aardige maar ook depressieve, vervelende en gemene opmerkingen tijdens de afgelopen vier jaar. Kortom, bedankt dat je altijd eerlijk tegen me was en me een spiegel voorhield.

Martine, Tineke, Sander, Viola, Mandy, Joris, Merijn, Mirjam, Francine, Eline, Luc, Eva, Jochen (CAPHRI), Lia en Esther (MHeNS), Joep, Pim en Dilana (FdP): ik wil jullie bedanken voor jullie vriendelijkheid, hulp, en steun de afgelopen jaren.

Kitty en Marga, vooral de laatste twee jaar van mijn promotie ben ik vaak even bij jullie komen kletsen. Ik hoop dat jullie onze gesprekken net zo leuk hebben ervaren dan ik. Ik wens jullie alleen nog maar mooie dingen voor de toekomst; jullie verdienen het!

Ine, Kim en Judith, bedankt dat jullie mij altijd van dienst wilde zijn bij het plannen van afspraken, het regelen van een buitenlands congres of als ik weer eens ruzie had met de faxmachine.

Ik wil hierbij ook de dames van de boekenclub bedanken. Onze boekenclub bestaat uit allemaal vrouwen die of bezig zijn met een promotie binnen CAPHRI of de promotie al hebben afgerond. Promoveren kan soms al aardig lastig zijn, maar dan ook nog eens elke maand een boek voor de boekenclub lezen dat regelmatig wel 500 bladzijdes of meer besloeg, was helemaal een uitdaging. En dan 'vergeten' we voor het gemak even dat de meeste van jullie ook nog mama zijn of zwanger zijn. De mate waarin we elke bijeenkomst over baby'tjes praatten hield dikwijls verband met het boek dat we gelezen hadden (teleurstellend boek $\rightarrow$ veel baby praat). Onze bijeenkomsten heb ik altijd erg gewaardeerd, mede door het lekkere eten. Hopelijk mogen er nog veel bijeenkomsten volgen!

Ten slotte wil ik twee collega's bedanken die tevens mijn paranimfen én vriendinnetjes zijn; Petra Erkens en Janaica Grispen. Beste Petra, de afgelopen drie jaar hebben we niet alleen een werkkamer maar ook lief en leed gedeeld. Verhalen over ons werk, Frank, Sven, onze families, onze weekenden, kleren, schoenen en kapsels; ze kwamen allemaal voorbij. Ik heb het delen van een kamer met jou als enorm fijn ervaren. Ik kan alleen maar hopen dat dit voor jou ook geldt. Beste Janaica, jij was degene die zorgde voor de 'stimmung' op tweede verdieping. Mocht ik eens geen zin hebben om te werken dan dacht ik gewoon aan het feit dat jij er ook zou zijn met je aanstekelijke lach en goede zin. Jouw aanwezigheid brengt een vrolijkheid met zich mee die je absoluut nooit moet verliezen! 


\section{Vrienden}

Als ik wel eens zeurde over mijn promotie traject zag ik mijn familie wel eens denken: 'Promoveren? Hoe stressvol kan dat nu werkelijk zijn als je 46 vrije dagen in het jaar hebt en een dag in de week thuis werkt'. Eigenlijk hebben jullie daar helemaal gelijk in.

Lieve Daan, misschien is niet alles in ons leven gelopen zoals we hadden verwacht, maar ik heb je vriendschap altijd enorm gewaardeerd.

Fieke, bedankt voor alle keren dat je onverwacht op de stoep stond omdat je even wilde komen kletsen. Ik hoop dat je dat in de toekomst blijft doen, ook nu je mama bent van een geweldig mooie meid.

Geert, vaak belde je Sven om te vragen of je nú een kop koffie kon komen drinken. Anderzijds belde Sven je vaker als hij weer hulp in de tuin nodig had. Als je op bezoek kwam zorgde je ervoor dat ik even mijn eigen ding kon doen; Sven en jij vermaakten elkaar wel. Ik heb in mijn leven nog nooit twee mannen gezien die zo kunnen roddelen!

Kirsten en Roy (en hun 2 honden en 3 katten!), onze gourmette avond zijn altijd lekker (gezellig). Ik hoop jullie nog vaak te mogen ontvangen/bezoeken.

Beste Sander (Tasma voor intimi), ik wil jou hier toch ook even bedanken voor alle keren dat jij en de rest van de jongens me mee op sleeptouw wilden nemen. Tevens bedankt voor alle keren dat je me laat winnen als we Wordfeud spelen!

\section{Familie}

Ik vind het erg jammer dat mijn beide grootouders mijn promotie niet meer kunnen meemaken. Ik had graag gewild dat ze hadden begrepen waar ik aan werkte. Echter, door hun harde werken tijdens een, niet altijd aangenaam leven, hebben ze de een goede basis gelegd voor hun kinderen en daardoor dus ook voor hun kleinkinderen. Mijn proefschrift draag ik dan ook op aan hen.

Martijn (Tinus voor intimi), ik wil je vooral bedanken voor het tweede en derde jaar tijdens mijn promotie. Ik keek altijd enorm uit naar vrijdag en zaterdag omdat we dan weer gezellig op stap gingen en shoarma konden eten. We zien elkaar nog steeds regelmatig, ook al is mijn leven nu iets burgerlijker geworden. Hoewel . . . een wedstrijd van Roda JC bezoeken en dan op de west tribune zitten, is mijn inziens alles behalve burgerlijk. Een bedankje ook naar de Danielle (mama van Tinus) die haar huis altijd openstelde voor zo veel jong volk.

Andy en Sammy, jullie wil ik bedanken voor alle leuke spelletjes avonden! Monopolie, Cluedo, Buzz alles was even leuk! Mogen er in de toekomst nog veel van deze avonden volgen! 
Hans, Gina en Joy, wij zijn altijd welkom bij jullie en niets lijkt jullie te veel moeite. Bedankt! Joy, wanneer kom je nu eens gezellig bij ons slapen?

Ondanks dat je vaak de wildste verhalen hoort over schoonfamilies en in het bijzonder over schoonmoeders, kan ik niet anders dan toegeven dat ik het enorm getroffen heb op dit gebied. Lieve schoonmoeder, bedankt voor alle leuke momenten de afgelopen jaren. Jouw liefde voor uit eten gecombineerd met mijn liefde voor eten, of dat nu uit of thuis is, heeft geleidt tot vele etentjes die altijd een welkome afleiding waren. Moge er nog vele volgen! Bedankt ook voor al die uren die je poetsend hebt doorgebracht in ons nieuwe huis toen ik in Washington werkte.

Lieve schone vader (zoals je jezelf vaker noemt) en Martha bedankt voor de keren dat we mochten komen eten op vrijdagavond (ondanks dat ik ze altijd op de kop kreeg als ik een halve pot mayonaise bij mijn frietjes at). Tevens bedankt voor alle hulp aan ons huis!

Het bezoeken van een festival is bij uitstek het beste middel om niet aan je werk te denken. Bennie en Ineke, bedankt voor alle leuke Pinkpop momenten. Ik zie ons nog genieten bij Bruce. En dit jaar komt hij weer! Zoë en Evaatje, hoe kun je een weekend beter beginnen dan je liefste nichtjes uit te nodigen om te komen logeren? Jullie zijn lieverdjes!

Papa en mama, doordat jullie altijd benadrukten hoe belangrijk (maar zeker niet hét allerbelangrijkst) een goede opleiding was, hebben jullie de fundamenten gelegd voor mijn opleiding als psycholoog en daardoor dus ook voor mijn promotie. Sanne, ondanks dat de inhoud en de vorm anders is, leveren we beiden een bijdrage aan de gezondheidszorg, iets dat ons beiden enorm interesseert.

Lieve Sven, ik wil jou bedanken voor je luisterend oor, je altijd aanwezige glimlach, je optimisme, de 'stemmetjes die je doet' (Sven kan heel goed een kermisattractie na doen!) om me op te vrolijken en je aandacht voor mij. Al deze factoren die zo kenmerkend zijn voor jou als mens zorgen ervoor dat ik me gesteund, speciaal en geliefd voel. Vriendjes? 


\section{ABOUT THE AUTHOR}



Sil Aarts was born on August 26, 1985 in Heerlen, the Netherlands. After completing her Gymnasium at Sint-Janscollege in Hoensbroek, she studied psychology at Maastricht University. Here she also obtained her master's degree in neuropsychology. During her master education Sil Aarts became aware of her special interest in statistical analyzing techniques. Before the end of her master education, she got the position of a $\mathrm{PhD}$ student at School for Public health and Primary Care (CAPHRI) and School for Mental Health and Neuroscience (MHeNS). Her PhD trajectory revolved around two main projects which were aimed at investigating the effects of multimorbidity on (neuro)psychological and cognitive outcome measures, taking into account sociodemographic and socioeconomic characteristics and on using innovative methods for analyzing large amounts of medical data. During her second and third year as a $\mathrm{PhD}$ student she was a $\mathrm{PhD}$ representative for CAPHRI, a position that she tried to fulfil with care. From September until December 2010, she worked at the National Institute on Aging, part of National Institutes of health in Bethesda, Maryland, USA.

At the end of 2011, she was offered a post-doctoral position at CAPHRI. However, at the start of 2012, she decided that it was time for something new. Hence, since the $27^{\text {th }}$ of February she is employed by Fontys as a teacher in scientific research.

Sil Aarts lives happily together with her hopefully soon to be husband, Sven and her rabbit Lady. Beyond her work she enjoys running, reading (for book club 'the Komaas') and learning new skills (every Tuesday she goes to French class with her mom) and travelling. She also loves watching sports and together with some friends (all male by the way) she regularly visits soccer matches of Roda JC (but is actually more of a FC Ajax fan). 

PUBLICATIONS

-167 - 

S. Aarts, B. Winkens, M. van den Akker. The insignifance of statistical significance. European Journal of General Practice. 2011 Dec 5 (Epub ahead of print).

S. Aarts, M. van den Akker, H. Bosma, F.E. Tan, F.R. Verhey, J.F. Metsemakers, M.P. van Boxtel. Pathologies chroniques uniques ou multiples: leurs effets sur la santé physique ou mentale sont-ils temporaires ou persistants?. Excercer. 2011;99(1):90-91.

S. Aarts, M. van den Akker, F.E. Tan, F.R. Verhey, J.F. Metsemakers, M.P. van Boxtel. Influence of multimorbidity on cognition in a normal aging population: a 12-year followup in the Maastricht Aging Study. International Journal of Geriatric Psychiatry. 2011;26:1046-1053.

S. Aarts, M. van den Akker M, F.E. Tan, F.R. Verhey, J.F. Metsemakers, M.P. van Boxtel. De invloed van multimorbiditeit op het cognitief functioneren. Huisarts en Wetenschap. 2011;54(3).

S. Aarts, M. van den Akker, K.J. Hajema, A.M. van Ingen, J.F. Metsemakers, F.R. Verhey FR, M.P. van Boxtel. Multimorbidity and its relation to subjective memory complaints in a large general population of older adults. International Psychogeriatrics. 2011;23(4):616624.

S. Aarts, M. van den Akker, F.E. Tan, F.R. Verhey, J.F. Metsemakers, M.P. van Boxtel. Multimorbidité et cognition: étude longitudinal dans une population âgée normale. Excercer. 2010;92(3):84-85.

S. Aarts, M. van den Akker, M.P. van Boxtel, J. Jolles, B. Winkens, J.F. Metsemakers. Diabetes mellitus type II as a risk factor for depression: a lower than expected risk in a general practice setting. European Journal of Epidemiology. 2009;24(10):641-648.

S. Aarts, M. van den Akker, F.E. Tan, F.R. Verhey, H. Bosma, J.F. Metsemakers, M.P. van Boxtel. The effect of single morbidity and multimorbidity on mental and physical health: temporary or persistent?. (under review).

S. Aarts, K.V. Patel, M.E. Garcia, M. van den Akker, F.R. Verhey, J.F. Metsemakers, M.P. van Boxtel, M. Jonsdottir, K. Siggeirsdottir, P.V. Jonsson, T.B. Harris, L.J. Launer. Cooccurrence of disability and multimorbidity with frailty: an examination of heterogeneity in the frail older population. (under review).

S. Aarts, M. van den Akker, F.R. Verhey, J.F. Metsemakers, M.P. van Boxtel, R. Vos. Patterns of multimorbidity: identifying psychiatric and somatic disease combinations using an exploratory analyzing approach. (under review). 


\section{Chapter 10}

S. Aarts, M. van den Akker, F.E. Tan, F.R. Verhey, H. Bosma, R. Vos, J.F. Metsemakers, M.P. van Boxtel. Exploring medical data to generate new hypotheses: an introduction to data and text mining techniques. (submitted).

R. Vos, S. Aarts, E. van Mulligen, J. Kors, F.R. Verhey, J.F. Metsemakers, M.P. van Boxtel, M. van den Akker. Searching for potentially new clusters of psychiatric and somatic diseases - knowledge discovery and text mining relating to patterns of multimorbidity in medical literature (Medline). (submitted). 